\title{
LOW MACH NUMBER LIMIT OF THE FULL NAVIER-STOKES EQUATIONS
}

\author{
THOMAS ALAZARD
}

\begin{abstract}
The low Mach number limit for classical solutions to the full Navier Stokes equations is here studied. The combined effects of large temperature variations and thermal conduction are accounted. In particular we consider general initial data. The equations lead to a singular problem, depending on a small scaling parameter, whose linearized is not uniformly well-posed. Yet, it is proved that the solutions exist and are uniformly bounded for a time interval which is independent of the Mach number Ma $\in(0,1]$, the Reynolds number $\operatorname{Re} \in[1,+\infty]$ and the Péclet number $\mathrm{Pe} \in[1,+\infty]$. Based on uniform estimates in Sobolev spaces, and using a Theorem of G. Métivier and S. Schochet 31, we next prove that the penalized terms converge strongly to zero. It allows us to rigorously justify, at least in the whole space case, the well-known computations given in the introduction of the P.-L. Lions' book [26].
\end{abstract}

\section{CONTENTS}

1. Introduction 1

2. Main results 7

3. Localization in the frequency space 11

4. Energy estimates for the linearized svstem 17

5. High frequency regime 30

6. Low frequency regime 38

7. Uniform stability 60

8. Decav of the local energy 63

Appendix A. Estimates for hvperbolic and parabolic svstems 66

References

\section{INTRODUCTION}

There are five key physical assumptions that dictate the nature of the low Mach number limit: the equations may be isentropic or non-isentropic; the fluid may be viscous or inviscid; the fluid may be an efficient or a poor thermal conductor; the domain may be bounded or unbounded; the temperature variations may be small or large. Yet, there are only two cases where the mathematical analysis of the low Mach number limit is well developed: first, in the isentropic regime [8, 9, 10, 11, 16, 27]; second, for inviscid and non heat-conductive fluids [1, 31, 32. 
Our goal is to start a rigorous analysis of the general case where the combined effects of large temperature variations and thermal conduction are accounted. As first anticipated in 28, it yields some new problems concerning the nonlinear coupling of the equations.

1.1. Setting the problem up. The full Navier-Stokes equations are:

$$
\left\{\begin{array}{l}
\partial_{t} \rho+\operatorname{div}(\rho v)=0, \\
\partial_{t}(\rho v)+\operatorname{div}(\rho v \otimes v)+\nabla P=\operatorname{div} \sigma \\
\partial_{t}(\rho e)+\operatorname{div}(\rho v e)+P \operatorname{div} v=\operatorname{div}(k \nabla \mathcal{T})+\sigma \cdot D v
\end{array}\right.
$$

where $\rho, v=\left(v^{1}, \ldots, v^{d}\right), P, e$ and $\mathcal{T}$ denote the fluid density, velocity, pressure, energy and temperature, respectively. We consider Newtonian gases with Lamé viscosity coefficients $\zeta$ and $\eta$, so that the viscous strain tensor $\sigma$ is given by

$$
\sigma:=2 \zeta D v+\eta \operatorname{div} v I_{d}
$$

where $2 D v=\nabla v+(\nabla v)^{t}$ and $I_{d}$ is the $d \times d$ identity matrix.

Considerable insight comes from being able to simplify the description of the governing equations (1.11) by introducing clever physical models and the use of judicious mathematical approximations. To reach this aim, a standard strategy is to introduce dimensionless numbers which determine the relative significance of competing physical processes taking place in moving fluids. Not only this allows to derive simplified equations of motion, but also reveals the central feature of the phenomenon considered.

In this paper we distinguish three dimensionless parameters:

$$
\varepsilon \in(0,1], \quad \mu \in[0,1] \text { and } \quad \kappa \in[0,1] .
$$

The first parameter $\varepsilon$ is the Mach number, namely the ratio of a characteristic velocity in the flow with the sound speed in the fluid. The parameters $\mu$ and $\kappa$ are essentially the inverses of the Reynolds and Péclet numbers, they measure the importance of viscosity and heat-conduction.

To rescale the equations, there are basically two approaches which are available. The first is to cast equations in dimensionless form by scaling every variable by its characteristic value [28, 34. The second is to consider one of the three changes of variables:

$$
\begin{aligned}
& t \rightarrow \varepsilon^{2} t, \quad x \rightarrow \varepsilon x, \quad v \rightarrow \varepsilon v, \quad \zeta \rightarrow \mu \zeta, \quad \eta \rightarrow \mu \eta, \quad k \rightarrow \kappa k, \\
& t \rightarrow \varepsilon t, \quad x \rightarrow x, \quad v \rightarrow \varepsilon v, \quad \zeta \rightarrow \varepsilon \mu \zeta, \quad \eta \rightarrow \varepsilon \mu \eta, \quad k \rightarrow \varepsilon \kappa k, \\
& t \rightarrow t, \quad x \rightarrow x / \varepsilon, \quad v \rightarrow \varepsilon v, \quad \zeta \rightarrow \varepsilon \mu \zeta, \quad \eta \rightarrow \varepsilon \mu \eta, \quad k \rightarrow \varepsilon^{2} \kappa k .
\end{aligned}
$$

See [26, 43] for comments on the first two changes of variables. The third one is related to large-amplitude high-frequency solutions (these rapid variations are anomalous oscillations in the context of nonlinear geometric optics [5]). 
Anyway, these two approaches both yield the same result. The full Navier-Stokes equations, written in a non dimensional way, are:

$$
\left\{\begin{array}{l}
\partial_{t} \rho+\operatorname{div}(\rho v)=0, \\
\partial_{t}(\rho v)+\operatorname{div}(\rho v \otimes v)+\frac{\nabla P}{\varepsilon^{2}}=\mu \operatorname{div} \sigma, \\
\partial_{t}(\rho e)+\operatorname{div}(\rho v e)+P \operatorname{div} v=\kappa \operatorname{div}(k \nabla \mathcal{T})+\varepsilon \mathcal{Q},
\end{array}\right.
$$

where $\mathcal{Q}:=\varepsilon^{\alpha} \mu \sigma \cdot D v$ with $\alpha \geqslant 0$ (namely, $\alpha=1$ for the first two changes of variables and $\alpha=0$ for the last one).

Our study is concerned with the analysis of the low Mach number limit for classical solutions to the full Navier Stokes equations (1.3) in the nonisentropic general case and for general initial data. In particular, the combined effects of large temperature variations and thermal conduction are accounted. We are interested in the limit $\varepsilon \rightarrow 0$ and in proving results that are independent of $\mu$ and $\kappa$. The analysis contains two parts. We first prove an existence and uniform boundedness result for a time interval independent of the parameters $\varepsilon, \mu$ and $\kappa$. We next study the behavior of the solutions when $\varepsilon$ tends to 0 .

Many results have been obtained in the past two decades about the justification of the incompressible limit - which is a special case of the low Mach number approximation. Concerning the Euler equations $(\mu=\kappa=0)$, the study began in earlies eighties with works of Klainerman and Majda [22, 23, Schochet [36], Isozaki [18, 19], Ukai [41, and others. As regards the isentropic Navier-Stokes equations $(\mu=1, \zeta$ and $\eta$ constants, $\rho=\rho(P))$, the mathematical analysis of the low Mach number limit has come of age since the pioneering works. Recent progress are presented in Danchin [8, 9, Desjardins and Grenier [10, Desjardins, Grenier, Lions and Masmoudi [11, Hoff [16] and Lions and Masmoudi [27. They are also two very interesting earlier results concerning the group method: Grenier [14] and Schochet 37.

Concerning the non-isentropic Euler equations having general initial data, Métivier and Schochet have recently proved a couple of theorems 31, 30, 32. that supersede a number of earlier results (a part of their study is extended in [1] to the boundary case). In particular they have proved the existence of classical solutions on a time interval independent of $\varepsilon$. The aim of this paper is precisely to start a rigorous analysis of the corresponding problems for the full Navier-Stokes equations.

The study of the low Mach number limit is a vast subject of which we have barely scratched the surface here. To fill in this gap we recommend Desjardins and Lin 12 and Gallagher 13] for well written survey papers. For the reader who wishes to learn more about the physics, P.-L. Lions [26], Majda 28 and Zeytounian 43, 44 are good places to start. Detailed historical accounts of the subject can be found in 34, along with a broad number of references for further reading. In connection to the stability analysis performed below, let us point out that the research of numerical algorithms valid for all flow speeds is a very active field [15, 24, 35, 42. 
1.2. Uniform stability. We consider classical solutions, that is solutions valued in the Sobolev spaces $H^{S}(\mathbb{D})$ with $s$ large enough, the domain $\mathbb{D}$ being either the whole space $\mathbb{R}^{d}$ or the torus $\mathbb{T}^{d}$. Our main result asserts that, for perfect gases, the classical solutions exist and are uniformly bounded for a time interval independent of $\varepsilon, \mu$ and $\kappa$. We mention that the case of general gases involved additional difficulties (see Remarks 1.4 and 1.6) and will be addressed in a separate paper.

We choose to work with the unknowns $P, v=\left(v^{1}, \ldots, v^{d}\right)$ and $\mathcal{T}$. In order to be closed, the system must to be augmented with two equations of state, prescribing the density $\rho$ and the energy $e$ as given functions of $P$ and $\mathcal{T}$. Here, we restrict ourselves to perfect gases so that

$$
P=R \rho \mathcal{T} \quad \text { and } \quad e=C_{V} \mathcal{T},
$$

where $R$ and $C_{V}$ are given positive constants. We begin by rewriting equations (1.3) in terms of $(P, v, \mathcal{T})$. Set $\gamma=1+R / C_{V}$. Performing linear algebra, it is found that

$$
\left\{\begin{array}{l}
\partial_{t} P+v \cdot \nabla P+\gamma P \operatorname{div} v=(\gamma-1) \kappa \operatorname{div}(k \nabla \mathcal{T})+(\gamma-1) \varepsilon \mathcal{Q}, \\
\rho\left(\partial_{t} v+v \cdot \nabla v\right)+\frac{\nabla P}{\varepsilon^{2}}=\mu \operatorname{div} \sigma, \\
\rho C_{V}\left(\partial_{t} \mathcal{T}+v \cdot \nabla \mathcal{T}\right)+P \operatorname{div} v=\kappa \operatorname{div}(k \nabla \mathcal{T})+\varepsilon \mathcal{Q} .
\end{array}\right.
$$

where $\rho=P /(R \mathcal{T})$. The equations (1.4) are supplemented with initial data:

$$
P_{\mid t=0}=P_{0}, \quad v_{\mid t=0}=v_{0} \quad \text { and } \quad \mathcal{T}_{\mid t=0}=\mathcal{T}_{0} .
$$

Finally, it is assumed that $\zeta, \eta$ and the coefficient of thermal conductivity $k$ are $C^{\infty}$ functions of the temperature $\mathcal{T}$, satisfying

$$
k(\mathcal{T})>0, \quad \zeta(\mathcal{T})>0 \quad \text { and } \quad \eta(\mathcal{T})+\zeta(\mathcal{T})>0
$$

Notation 1.1. Hereafter, $A$ denotes the set of adimensioned parameters:

$$
A:=\{a=(\varepsilon, \mu, \kappa) \mid \varepsilon \in(0,1], \mu \in[0,1], \kappa \in[0,1]\} .
$$

Theorem 1.2. Let $d \geqslant 1$ and $\mathbb{D}$ denotes either the whole space $\mathbb{R}^{d}$ or the torus $\mathbb{T}^{d}$. Consider an integer $\mathrm{s}>1+d / 2$. For all positive $\underline{P}, \underline{\mathcal{T}}$ and $M_{0}$, there is a positive time $T$ such that for all $a=(\varepsilon, \mu, \kappa) \in A$ and all initial data $\left(P_{0}^{a}, v_{0}^{a}, \mathcal{T}_{0}^{a}\right)$ such that $P_{0}^{a}$ and $\mathcal{T}_{0}^{a}$ take positive values and such that

$$
\varepsilon^{-1}\left\|P_{0}^{a}-\underline{P}\right\|_{H^{s+1}(\mathbb{D})}+\left\|v_{0}^{a}\right\|_{H^{s+1}(\mathbb{D})}+\left\|\mathcal{T}_{0}^{a}-\underline{\mathcal{T}}\right\|_{H^{s+1}(\mathbb{D})} \leqslant M_{0},
$$

the Cauchy problem for (1.4)-(1.5) has a unique solution $\left(P^{a}, v^{a}, \mathcal{T}^{a}\right)$ such that $\left(P^{a}-\underline{P}, v^{a}, \mathcal{T}^{a}-\underline{\mathcal{T}}\right) \in C^{0}\left([0, T] ; H^{s+1}(\mathbb{D})\right)$ and such that $P^{a}$ and $\mathcal{T}^{a}$ take positive values. In addition there exists a positive $M$, depending only on $M_{0}, \underline{P}$ and $\underline{\mathcal{T}}$, such that

$\sup _{a \in A} \sup _{t \in[0, T]}\left\{\varepsilon^{-1}\left\|P^{a}(t)-\underline{P}\right\|_{H^{s}(\mathbb{D})}+\left\|v^{a}(t)\right\|_{H^{s}(\mathbb{D})}+\left\|\mathcal{T}^{a}(t)-\underline{\mathcal{T}}\right\|_{H^{s}(\mathbb{D})}\right\} \leqslant M$. 
The main obstacle is that the equations leads to a singular problem, depending on the small scaling parameter $\varepsilon$, whose linearized is not uniformly well-posed in Sobolev spaces. In other words, since we consider large temperature variations, the problem is linearly (uniformly) unstable (see [31] for comments on this instability). Therefore, we cannot obtain the nonlinear energy estimates by differentiating the equations nor by localizing in the frequency space by means of Littlewood-Paley operators. In particular, the technical aspects are different from those present in the previous studies of the Cauchy problem for strong solutions of (1.1). ${ }^{1}$

Remark 1.3. i. We will prove a more precise result which, in particular, exhibits some new smoothing effects for div $v$ and $\nabla P$ (see Theorem [2.7). ii. General initial data are here considered, and allow for large density and temperature variations. The hypothesis $P_{0}^{a}(x)-\underline{P}=\mathcal{O}(\varepsilon)$ is the natural scaling to balance the acoustic components, see (1.6) and 9, 22, 28, 31.

iii. One technical reason why we are uniquely interested in the whole space $\mathbb{R}^{d}$ or the Torus $\mathbb{T}^{d}$ is that we will make use of the Fourier transform tools. A more serious obstacle is that, in the boundary case, there should be boundary layers to analyze [3]. For the Euler equations (that is $\mu=\kappa=0$ ), however, Theorem 1.2 remains valid in the boundary case [1.

Before leaving this paragraph, let us say some words about the difficulties involved in the proof of Theorems 1.2. After changes of variables (given in $\$ 2.4$ ), we are led to study a mixed hyperbolic/parabolic system of nonlinear equations of the form:

$$
\left\{\begin{array}{l}
g_{1}(\theta, \varepsilon p)\left(\partial_{t} p+v \cdot \nabla p\right)+\frac{1}{\varepsilon} \operatorname{div} v-\frac{\kappa}{\varepsilon} \chi_{1}(\varepsilon p) \operatorname{div}(\beta(\theta) \nabla \theta)=\Upsilon_{1}, \\
g_{2}(\theta, \varepsilon p)\left(\partial_{t} v+v \cdot \nabla v\right)+\frac{1}{\varepsilon} \nabla p-\mu B_{2}(\theta, \varepsilon p) v=0, \\
g_{3}(\theta, \varepsilon p)\left(\partial_{t} \theta+v \cdot \nabla \theta\right)+\operatorname{div} v-\kappa \chi_{3}(\varepsilon p) \operatorname{div}(\beta(\theta) \nabla \theta)=\varepsilon \Upsilon_{3},
\end{array}\right.
$$

where $B_{2}$ is a second order elliptic differential operator, and $\Upsilon_{i}(i=1,3)$ are of no consequence.

One of the main differences between the Euler equations [1, 31] and the full equations is the following. When $\kappa=0$, it is typically easy to obtain $L^{2}$ estimates uniform in $\varepsilon$ by a simple integration by parts in which the large terms in $1 / \varepsilon$ cancel out (see 22, 31]). In sharp contrast (as observed in [28]), when $\kappa \neq 0$ and the initial temperature variations are large, the problem is more involved because the penalization operator is no longer skew-symmetric. Several difficulties also specifically arise for proving estimates that are independent of $\mu$ and $\kappa$.

Another main feature of the system (1.6) is that $g_{1}$ and $g_{2}$ depend on $\theta$. As a consequence $\nabla g_{1}$ and $\nabla g_{2}$ are of typical size $\mathcal{O}(1)$. The system (1.6)

\footnotetext{
${ }^{1}$ The latter problem has been widely studied. Starting from [29] and culminating in 6] 7] which investigate global strong solutions in spaces invariant by the scaling (1.2) with $\mu=\kappa=1$, following the approach initiated by Fujita and Kato.
} 
does not enter into the classical framework of singular limit [14, 37] because of this strong coupling between the short time-scale and the fast time-scale. That is why we cannot derive estimates in Sobolev norm by standards methods using differentiation of the equations. In this regard, an heuristic argument is worth emphasis: For non-isentropic fluids, the pressure depends on the density as well as the temperature. Thus, as the Mach number goes to zero, the fact the variation in pressure converges to zero does not preclude large temperature fluctuations (provided that they are compensated by density fluctuations of opposite sign). Yet, even small perturbations of the density might result in non-small velocity variations.

We conclude this part with a remark concerning general gases.

Remark 1.4. For general equations of state, we are led to study systems having the form (1.6) where the coefficients $\chi_{1}$ and $\chi_{3}$ depend also on $\theta$. As a consequence, the singular operator is nonlinear. Some computations indicate that there are no $L^{2}$ estimates uniform with respect to $\varepsilon$.

1.3. The low Mach number limit. We now turn to consideration of the limit of solutions of (1.3) in $\mathbb{R}^{d}$ as the Mach number $\varepsilon$ goes to 0 . The purpose of the low Mach number approximation is to justify that the compression due to pressure variations can be neglected. This is a common assumption that is made when discussing the fluid dynamics of highly subsonic flows. In particular, provided the sound propagation is adiabatic, it is the same as saying that the flow is incompressible. On sharp contrast, this is no longer true if the combined effect of large temperature variations and heat conduction is accounted. Indeed, going back to (1.4) we compute that, formally, the limit system reads

$$
\left\{\begin{array}{l}
\gamma \underline{P} \operatorname{div} v=(\gamma-1) \kappa \operatorname{div}(k \nabla \mathcal{T}), \\
\rho\left(\partial_{t} v+v \cdot \nabla v\right)+\nabla \pi=\mu \operatorname{div} \sigma, \\
\rho C_{P}\left(\partial_{t} \mathcal{T}+v \cdot \nabla \mathcal{T}\right)=\kappa \operatorname{div}(k \nabla \mathcal{T}),
\end{array}\right.
$$

where $\rho=\underline{P} /(R \mathcal{T}), C_{P}=\gamma C_{V}$ and, in keeping with the notations of Theorem[1.2 $\underline{P}$ denotes the constant value of the pressure at spatial infinity.

Theorem 1.5. Fix $\mu \in[0,1]$ and $\kappa \in[0,1]$. Assume that $\left(P^{\varepsilon}, v^{\varepsilon}, \mathcal{T}^{\varepsilon}\right)$ satisfy (1.4) and

$$
\sup _{\varepsilon \in(0,1]} \sup _{t \in[0, T]}\left\|\varepsilon^{-1}\left(P^{\varepsilon}(t)-\underline{P}\right)\right\|_{H^{s}}+\left\|v^{\varepsilon}(t)\right\|_{H^{s}}+\left\|\mathcal{T}^{\varepsilon}(t)-\underline{\mathcal{T}}\right\|_{H^{s}}<+\infty,
$$

for some fixed time $T>0$, reference states $\underline{P}, \underline{\mathcal{T}}$ and index s large enough. Suppose in addition that the initial data $\mathcal{T}_{\mid t=0}^{\varepsilon}-\underline{\mathcal{T}}$ is compactly supported. Then, for all $s^{\prime}<s$, the pressure variations $\varepsilon^{-1}\left(P^{\varepsilon}-\underline{P}\right)$ converges strongly to 0 in $L^{2}\left(0, T ; H_{l o c}^{s^{\prime}}\left(\mathbb{R}^{d}\right)\right)$. Moreover, for all $s^{\prime}<s,\left(v^{\varepsilon}, \mathcal{T}^{\varepsilon}\right)$ converges strongly in $L^{2}\left(0, T ; H_{\text {loc }}^{s^{\prime}}\left(\mathbb{R}^{d}\right)\right)$ to a limit $(v, \mathcal{T})$ satisfying the limit system (1.7). 
Note that the convergence is not uniform in time for the oscillations on the acoustic time-scale prevent the convergence of the solutions on a small initial layer in time. The key to proving this convergence result is to prove the decay to zero of the local energy of the acoustic waves. To do so we will consider general systems which include (1.6) as a special case. In particular, the analysis of the general systems considered below should apply for the study of the low Mach number combustion as described in 28. We mention that, in view of [1, it seems possible to consider the same problem for exterior domains (which is interesting for aeroacoustic [44]). Yet, we will not address this question. The results proved in [3, 32 indicate that the periodic case involved important additional phenomena.

Remark 1.6. For perfect gases, the limit constraint is linear in the following sense: it is of the form $\operatorname{div} v_{e}=0$ with $v_{e}:=v-C^{t e} \kappa k \nabla \mathcal{T}$. In sharp contrast, for general gases the constraint is nonlinear. Indeed, it reads $\operatorname{div} v=f(\underline{P}, \mathcal{T}) \kappa \operatorname{div}(k \nabla \mathcal{T})$. As a consequence, it is not immediate that, in this case, the corresponding Cauchy problem for (1.7) is well posed.

\section{MAIN RESUlts}

We will see in 2.4 below that one can transform the equations (1.4) into a system of the form

$$
\left\{\begin{array}{l}
g_{1}(\theta, \varepsilon p)\left(\partial_{t} p+v \cdot \nabla p\right)+\frac{1}{\varepsilon} \operatorname{div} v-\frac{\kappa}{\varepsilon} B_{1}(\theta, \varepsilon p) \theta=\Upsilon_{1}, \\
g_{2}(\theta, \varepsilon p)\left(\partial_{t} v+v \cdot \nabla v\right)+\frac{1}{\varepsilon} \nabla p-\mu B_{2}(\theta, \varepsilon p) v=0, \\
g_{3}(\theta, \varepsilon p)\left(\partial_{t} \theta+v \cdot \nabla \theta\right)+\operatorname{div} v-\kappa B_{3}(\theta, \varepsilon p) \theta=\varepsilon \Upsilon_{3},
\end{array}\right.
$$

where the unknown $(p, v, \theta)$ is a function of the variables $(t, x) \in \mathbb{R} \times \mathbb{D}$ with values in $\mathbb{R} \times \mathbb{R}^{d} \times \mathbb{R}$. Recall that $\mathbb{D}$ denotes either the whole space $\mathbb{R}^{d}$ or the torus $\mathbb{T}^{d}$. Moreover, the coefficients $g_{i}, i=1,2,3$, are real-valued and the $B_{i}$ 's are second order differential operators given by:

$$
\begin{aligned}
& B_{1}(\theta, \varepsilon p):=\chi_{1}(\varepsilon p) \operatorname{div}(\beta(\theta) \nabla \cdot), \\
& B_{2}(\theta, \varepsilon p):=\chi_{2}(\varepsilon p) \operatorname{div}(2 \zeta(\theta) D \cdot)+\chi_{2}(\varepsilon p) \nabla(\eta(\theta) \operatorname{div} \cdot), \quad 2 D:=\nabla+(\nabla \cdot)^{t}, \\
& B_{3}(\theta, \varepsilon p):=\chi_{3}(\varepsilon p) \operatorname{div}(\beta(\theta) \nabla \cdot) .
\end{aligned}
$$

Finally, $\Upsilon_{i}:=\chi_{i}(\theta, \varepsilon p) F(\theta, \sqrt{\mu} \nabla v)$ where $F \in C^{\infty}$ is such that $F(0)=0$.

\subsection{Structural assumptions.}

Assumption 2.1. To avoid confusion, denote by $(\vartheta, \wp) \in \mathbb{R}^{2}$ the place holder of the unknown $(\theta, \varepsilon p)$.

(A1) The $g_{i}$ 's, $i=1,2,3$, are $C^{\infty}$ positive functions of $(\vartheta, \wp) \in \mathbb{R}^{2}$.

(A2) The coefficients $\beta$ and $\zeta$ are $C^{\infty}$ positive functions of $\vartheta \in \mathbb{R}$, and $\eta$ is a $C^{\infty}$ function of $\vartheta \in \mathbb{R}$ satisfying $\eta(\vartheta)+\zeta(\vartheta)>0$.

(A3) The coefficients $\chi_{i}, i=1,2,3$, are $C^{\infty}$ positive functions of $\wp \in \mathbb{R}$. Moreover, for all $\wp \in \mathbb{R}$, there holds $\chi_{1}(\wp)<\chi_{3}(\wp)$. 
Assumption 2.2. Use the notation $\mathrm{d} f=(\partial f / \partial \vartheta) \mathrm{d} \vartheta+(\partial f / \partial \wp) \mathrm{d} \wp$. There exists two $C^{\infty}$ diffeomorphisms $\mathbb{R}^{2} \ni(\vartheta, \wp) \mapsto(S(\vartheta, \wp), \wp) \in \mathbb{R}^{2}$ and $\mathbb{R}^{2} \ni$ $(\vartheta, \wp) \mapsto(\vartheta, \varrho(\vartheta, \wp)) \in \mathbb{R}^{2}$ such that $S(0,0)=\varrho(0,0)=0$ and

$$
\mathrm{d} S=g_{3} \mathrm{~d} \vartheta-g_{1} \mathrm{~d} \wp \quad \text { and } \quad \mathrm{d} \varrho=-\frac{\chi_{1}}{\chi_{3}} g_{3} \mathrm{~d} \vartheta+g_{1} \mathrm{~d} \wp
$$

Brief discussion of the hypotheses.

(i) The main hypothesis in Assumption 2.1 is the inequality $\chi_{1}<\chi_{3}$. It plays a crucial role for the purpose of proving $L^{2}$ estimates (see the section 4 and especially the study of the simplified system (4.5)). Moreover, given the assumption $\beta(\vartheta)>0$, it ensures that the operator $B_{1}(\theta, 0)-B_{3}(\theta, 0)$ [which appears in the last equation of the limit system given below in [2.3)] is positive. Which means nothing but the fact that the limit temperature evolves according to the standard equation of heat diffusion!

(ii) The identities given in (2.2) are compatibility conditions between the penalization operator and the viscous perturbation. The reason for introducing $S$ [resp. @] will be clear in $\$ 6.5$ [resp. \$7.1].

2.2. Uniform stability result. Given $0 \leqslant t \leqslant T$, a normed space $X$ and a function $\varphi$ defined on $[0, T] \times \mathbb{D}$ with values in $X$, we denote by $\varphi(t)$ the function $\mathbb{D} \ni x \mapsto \varphi(t, x) \in X$. The usual Sobolev spaces are denoted $H^{\sigma}(\mathbb{D})$. Recall that, when $\mathbb{D}=\mathbb{R}^{d}$, they are equipped with the norms

$$
\|u\|_{H^{\sigma}}^{2}:=(2 \pi)^{-d} \int_{\mathbb{R}^{d}}\langle\xi\rangle^{2 \sigma}|\widehat{u}(\xi)|^{2} d \xi
$$

where $\widehat{u}$ is the Fourier transform of $u$ and $\langle\xi\rangle:=\left(1+|\xi|^{2}\right)^{1 / 2}$. As concerns the case $\mathbb{D}=\mathbb{T}^{d}$, replace the integrals in $\xi \in \mathbb{R}^{d}$ by sums on $k \in \mathbb{Z}^{d}$.

Let us introduce a bit of notation we use continually in the sequel.

Notation 2.3. Given $\sigma \in \mathbb{R}$ and $\varrho \geqslant 0$, set $\|u\|_{H_{\varrho}^{\sigma}}:=\|u\|_{H^{\sigma-1}}+\varrho\|u\|_{H^{\sigma}}$.

Recall that $A:=\{(\varepsilon, \mu, \kappa) \mid \varepsilon \in(0,1], \mu \in[0,1], \kappa \in[0,1]\}$. The norm that we will control is the following.

Definition 2.4. Let $T>0, \sigma \geqslant 0, a=(\varepsilon, \mu, \kappa) \in A$ and set $\nu:=\sqrt{\mu+\kappa}$. The space $\mathcal{H}_{a}^{\sigma}(T)$ consists of these functions $(p, v, \theta)$ defined on $[0, T] \times \mathbb{D}$ with values in $\mathbb{R} \times \mathbb{R}^{d} \times \mathbb{R}$ and such that $\|(p, v, \theta)\|_{\mathcal{H}_{a}^{\sigma}(T)}<+\infty$, where

$$
\begin{aligned}
& \|(p, v, \theta)\|_{\mathcal{H}_{a}^{\sigma}(T)}:=\sup _{t \in[0, T]}\left\{\|(p(t), v(t))\|_{H_{\varepsilon \nu}^{\sigma+1}}+\|\theta(t)\|_{H_{\nu}^{\sigma+1}}\right\}+ \\
& +\left(\int_{0}^{T} \mu\|\nabla v\|_{H_{\varepsilon \nu}^{\sigma+1}}^{2}+\kappa\|\nabla \theta\|_{H_{\nu}^{\sigma+1}}^{2}+\kappa\|\operatorname{div} v\|_{H^{\sigma}}^{2}+(\mu+\kappa)\|\nabla p\|_{H^{\sigma}}^{2} d t\right)^{\frac{1}{2}} .
\end{aligned}
$$

Similarly, the space $\mathcal{H}_{a, 0}^{\sigma}$ consists of these functions $(p, v, \theta)$ defined on $\mathbb{D}$ with values in $\mathbb{R} \times \mathbb{R}^{d} \times \mathbb{R}$ and such that $\|(p, v, \theta)\|_{\mathcal{H}_{a, 0}^{\sigma}}<+\infty$, where

$$
\|(p, v, \theta)\|_{\mathcal{H}_{a, 0}^{\sigma}}:=\|(p, v)\|_{H_{\varepsilon \nu}^{\sigma+1}}+\|\theta\|_{H_{\nu}^{\sigma+1}} \quad \text { with } \quad \nu:=\sqrt{\mu+\kappa} .
$$


Remark 2.5. One may not expect at first to have to take these norms into account, but they come up on their own in 4 Moreover, not only the norms, but also the spaces depend on $a$ (since we allow $\mu=0$ or $\kappa=0$ ).

Notation 2.6. Given a normed space $X$ and a non-negative $M$, we denote by $B(X ; M)$ the ball of center 0 and radius $M$ in $X$.

Here is our main result. In the context of Assumptions 2.1 2.2 we prove that the solutions of (2.1) exist and are uniformly bounded for a time interval which is independent of $a \in A$.

Theorem 2.7. Suppose that system (2.1) satisfies Assumptions [2.1-2.2, and let $d \geqslant 1$. For all integer $s>1+d / 2$ and for all positive $M_{0}$, there exists a positive $T$ and a positive $M$ such that for all $a \in A$ and all initial data in $B\left(\mathcal{H}_{a, 0}^{\mathrm{S}} ; M_{0}\right)$, the Cauchy problem for (2.1) has a unique classical solution in $B\left(\mathcal{H}_{a}^{s}(T) ; M\right)$.

The proof of this result will occupy us till 97.2 The crucial part is to obtain estimates in Sobolev norms that are independent of $a \in A$. Notable technical aspects include the proof of an energy estimate for linearized equations [see Theorem 4.3 and the use of new tools to localize in the frequency space [see Propositions 3.2 and 3.5 . With these results in hands, we begin in $\$ 5$ by analyzing the high frequency regime. The rest of the analysis is devoted to the proof of low frequencies estimates. We mention that we do not need specific estimates for medium frequencies.

Remark 2.8. Up to numerous changes, a close inspection of the proof of Theorem 2.7 indicates that, in fact: for all $M>M_{0}>0$, there exists $T>0$ such that for all $a \in A$ and all initial data in $B\left(\mathcal{H}_{a, 0}^{s} ; M_{0}\right)$ the Cauchy problem for (2.1) has a unique classical solution in $B\left(\mathcal{H}_{a}^{\mathrm{s}}(T) ; M\right)$.

2.3. Convergence toward the solution of the limit system. We now turn to considering the behavior of the solutions of (2.1) in $\mathbb{R}^{d}$ as the Mach number $\varepsilon$ tends to zero. Fix $\mu$ and $\kappa$ and consider a family of solutions of system (2.1), $\left(p^{\varepsilon}, v^{\varepsilon}, \theta^{\varepsilon}\right)$. It is assumed to be bound in $C\left([0, T] ; H^{\sigma}\left(\mathbb{R}^{d}\right)\right)$ with $\sigma$ large enough and $T>0$. Strong compactness of $\theta^{\varepsilon}$ is clear from uniform bounds for $\partial_{t} \theta^{\varepsilon}$. For the sequence $\left(p^{\varepsilon}, v^{\varepsilon}\right)$, however, the uniform bounds imply only weak compactness, insufficient to insure that the limits satisfies the limit equations. We remedy this by proving that the penalized terms converge strongly to zero.

Theorem 2.9. Suppose that the system (2.1) satisfies Assumption [2.1]. Fix $\mu \in[0,1]$ and $\kappa \in[0,1]$, and let $d \geqslant 1$. Assume that $\left(p^{\varepsilon}, v^{\varepsilon}, \theta^{\varepsilon}\right)$ satisfy (2.1) and are uniformly bounded in $\mathcal{H}_{(\varepsilon, \mu, \kappa)}^{s}(T)$ for some fixed $T>0$ and $s>4+d / 2$. Suppose that the initial data $\theta^{\varepsilon}(0)$ converge in $H^{s}\left(\mathbb{R}^{d}\right)$ to a function $\theta_{0}$ decaying sufficiently rapidly at infinity in the sense that $\langle x\rangle^{\delta} \theta_{0} \in H^{s}\left(\mathbb{R}^{d}\right)$ for some given $\delta>2$, where $\langle x\rangle:=\left(1+x^{2}\right)^{1 / 2}$.

Then, for all index $s^{\prime}<s, p^{\varepsilon} \rightarrow 0$ strongly in $L^{2}\left(0, T ; H_{l o c}^{s^{\prime}}\left(\mathbb{R}^{d}\right)\right)$ and $\operatorname{div} v^{\varepsilon}-\chi_{1}\left(\varepsilon p^{\varepsilon}\right) \operatorname{div}\left(\beta\left(\theta^{\varepsilon}\right) \nabla \theta^{\varepsilon}\right) \rightarrow 0$ strongly in $L^{2}\left(0, T ; H_{l o c}^{s^{\prime}-1}\left(\mathbb{R}^{d}\right)\right)$. 
The proof is given in $\$ 8$ It is based on a Theorem of Métivier and Schochet [31, which we describe in Theorem 8.3] about the decay to zero of the local energy for a class of wave operators with time dependent coefficients.

We have just seen that $p^{\varepsilon}$ converges to 0 . The following result states that $\left(v^{\varepsilon}, \theta^{\varepsilon}\right)$ converges toward the solution of the limit system.

Theorem 2.10. Using the same assumptions and notations as in Theorem [2.9, $\left(v^{\varepsilon}, \theta^{\varepsilon}\right)$ converges weakly in $L^{\infty}\left(0, T ; H^{S}\left(\mathbb{R}^{d}\right)\right)$ and strongly in $L^{2}\left(0, T ; H_{\text {loc }}^{s^{\prime}}\left(\mathbb{R}^{d}\right)\right)$ for all $s^{\prime}<s$ to a limit $(v, \theta)$ satisfying

$$
\left\{\begin{array}{l}
\operatorname{div} v=\kappa \chi_{1}(0) \operatorname{div}(\beta(\theta) \nabla \theta), \\
g_{2}(\theta, 0)\left(\partial_{t} v+v \cdot \nabla v\right)+\nabla \pi-\mu B_{2}(\theta, 0) v=0 \\
g_{3}(\theta, 0)\left(\partial_{t} \theta+v \cdot \nabla \theta\right)-\kappa\left(\chi_{3}(0)-\chi_{1}(0)\right) \operatorname{div}(\beta(\theta) \nabla \theta)=0
\end{array}\right.
$$

for some $\pi$ which can be chosen such that $\nabla \pi \in C^{0}\left([0, T] ; H^{s-1}\left(\mathbb{R}^{d}\right)\right)$.

Given Theorem 2.9 the proof of Theorem 2.10 follows from a close inspection of the proof of Theorem 1.5 in 31, and so will be omitted.

2.4. Changes of variables. To understand the role of the thermodynamics we rewrite equations (1.3) in terms of the pressure fluctuations $p$, velocity $v$ and temperature fluctuations $\theta$; where $p$ and $\theta$ are defined by

$$
P=\underline{P} e^{\varepsilon p}, \quad \mathcal{T}=\underline{\mathcal{T}} e^{\theta} \quad \text { or } \quad p:=\frac{1}{\varepsilon} \log \left(\frac{P}{P}\right), \quad \theta:=\log \left(\frac{\mathcal{T}}{\mathcal{T}}\right),
$$

where $\underline{P}$ and $\underline{\mathcal{T}}$ are given by the statement of Theorem 1.2 .

We can convert the pressure and temperature evolution equations into evolution equations for the fluctuations $p$ and $\theta$. Starting from (1.4), it is accomplished most readily by logarithmic differentiation $\left(\partial_{t, x} P=\varepsilon P \partial_{t, x} p\right.$ and $\left.\partial_{t, x} \mathcal{T}=\mathcal{T} \partial_{t, x} \theta\right)$. By so doing it is found that $(p, v, \theta)$ satisfies

$$
\left\{\begin{array}{l}
P\left(\partial_{t} p+v \cdot \nabla p\right)+\frac{\gamma P}{\varepsilon} \operatorname{div} v-\frac{(\gamma-1) \kappa}{\varepsilon} \operatorname{div}(k \mathcal{T} \nabla \theta)=(\gamma-1) \mathcal{Q}, \\
\frac{P}{R \mathcal{T}}\left(\partial_{t} v+v \cdot \nabla v\right)+\frac{P}{\varepsilon} \nabla p-\mu \operatorname{div}(2 \zeta D v)+\nabla(\eta \operatorname{div} v)=0 \\
\frac{C_{V} P}{R}\left(\partial_{t} \theta+v \cdot \nabla \theta\right)+P \operatorname{div} v-\kappa \operatorname{div}(k \mathcal{T} \nabla \theta)=\varepsilon \mathcal{Q} .
\end{array}\right.
$$

Therefore, the system (2.1) includes (2.5) as a special case where $g_{1}^{*}:=\frac{1}{\gamma}, g_{2}^{*}:=\frac{1}{R \mathcal{T}}, g_{3}^{*}:=\frac{C_{V}}{R}, \chi_{1}^{*}:=\frac{\gamma-1}{\gamma P}, \chi_{2}^{*}=\chi_{3}^{*}:=\frac{1}{P}, \beta^{*}=k(\mathcal{T}) \mathcal{T}$, where the $*$ indicates that the functions are evaluated at $(\theta, \varepsilon p)$. Moreover, for $i=1,3, \Upsilon_{i}:=\chi_{i}^{*} F(\theta, \sqrt{\mu} \nabla v)$ where $F(\theta, \sqrt{\mu} \nabla v):=\mathcal{Q}$ is as in (1.3).

We easily verify that the Assumptions 2.122.2 are satisfied in this case. Hence, Theorem 1.2 as stated in the introduction is now a consequence of Theorem 2.7 since $P$ and $\mathcal{T}$ (given by (2.4)) are obviously positive functions and since $\|\cdot\|_{C^{0}\left([0, T] ; H^{s}(\mathbb{D})\right)} \leqslant K\|\cdot\|_{\mathcal{H}_{a}^{s}(T)}$ for some constant $K$ independent of $a$. Similarly, Theorem 1.5 follows from Theorem 2.9 and Theorem 2.10. 


\section{LOCALIZATION IN THE FREQUENCY SPACE}

We now develop the analysis needed to localize in the frequency space. The first paragraph is a review consisting of various notations which serve as the requested background of what follows. The core of this section is $\S \S 3.23 .3$ in which we prove two technical ingredients needed to localize in the low frequency region. This will not be used before Section 6] and could be omitted before the reader gets there.

3.1. Preliminaries. To fix matters, in this section we work on the whole space $\mathbb{R}^{d}$, yet all the results are valid mutatis mutandis in the Torus $\mathbb{T}^{d}$. All functions are assumed to be complex valued unless otherwise specified. The notation $d$ always refers to the dimension, $\sigma_{0}$ always refers to a real number strictly greater than $d / 2$, and $h$ stands for a small parameter.

From now we use $K$ to denote a generic constant whose value may change from line to line in the text. It always stand for a constant independent of $h$ (whenever $h$ can play a role). Within the proofs, we use the notation $A \lesssim B$ to say that $A \leqslant K B$ for such a constant $K$.

We will often write the Sobolev spaces $H^{\sigma}\left(\mathbb{R}^{d}\right)$ as $H^{\sigma}$. Given two normed vector spaces $X_{1}$ and $X_{2}, \mathcal{L}\left(X_{1}, X_{2}\right)$ denotes the space of bounded operators from $X_{1}$ to $X_{2}$. We denote by $\|\cdot\|_{X_{1} \rightarrow X_{2}}$ its norm and $\mathcal{L}(X)$ is a shorthand notation for $\mathcal{L}(X, X)$.

Recall the notation

$$
\langle\xi\rangle:=\left(1+|\xi|^{2}\right)^{1 / 2}
$$

A function $q$ belongs to the class $S^{m}$ ( $m$ is a given real number) if $q(\xi)$ is a $C^{\infty}$ function of $\xi \in \mathbb{R}^{d}$ and satisfies the differential inequalities $\left|\partial_{\xi}^{\alpha} q(\xi)\right| \leqslant$ $Q_{\alpha}\langle\xi\rangle^{m-|\alpha|}$, for all $\xi \in \mathbb{R}^{d}$ and for all multi-indices $\alpha \in \mathbb{N}^{d}$. Such a function is called a symbol. With the best constant $Q_{\alpha}$ as semi-norms, $S^{m}$ is a Fréchet space. Given a symbol $q \in S^{m}$, the Fourier multiplier associated to $q$ is given by the operator $\mathcal{Q}$ acting on tempered distribution $u$ by $\widehat{\mathcal{Q} u}:=q \widehat{u}$. For $m, k \in \mathbb{R}$, let $\Lambda_{k}^{m}$ denotes the Fourier multiplier with symbol $\langle k \xi\rangle^{m}$. Put another way:

$$
\Lambda_{k}^{m}=\left(I-k^{2} \Delta\right)^{m / 2},
$$

where $I$ denotes the identity operator.

We now introduce the first of two families of operators which are used in the sequel to localize in the frequency space. Let $\jmath$ be a $C^{\infty}$ function of $\xi \in \mathbb{R}^{d}$, satisfying

$$
0 \leqslant \jmath \leqslant 1, \quad \jmath(\xi)=1 \text { for }|\xi| \leqslant 1, \quad \jmath(\xi)=0 \text { for }|\xi| \geqslant 2, \quad \jmath(\xi)=\jmath(-\xi) .
$$

Set $\jmath_{h}(\xi)=\jmath(h \xi)$, for $0 \leqslant h \leqslant 1$ and $\xi \in \mathbb{R}^{d}$; so that $\jmath_{h}$ is supported in the ball of radius $2 / h$ about the origin. Then we define $J_{h}$ as the Fourier multiplier with symbol $\jmath_{h}$ :

$$
J_{h}=\underset{11}{\jmath}\left(h D_{x}\right) .
$$


Let us make a series of remarks on $J_{h}$. The operator $J_{h}$ is self-adjoint since $\jmath_{h}$ is a real-valued function. Using that $\jmath_{h}$ is even, we deduce that $J_{h} u$ is real-valued for any real-valued tempered distribution $u$. They are smoothing operators, and the family $\left\{J_{h} \mid 0<h \leqslant 1\right\}$ is an approximate identity. In particular, we will often use the simple observation that for all $r \geqslant 0$, there exists a non-negative constant $K$ so that for all $h \in(0,1]$ and $\sigma \in \mathbb{R}$, one has

$$
\left\|J_{h}\right\|_{H^{\sigma} \rightarrow H^{\sigma+r}} \leqslant \frac{K}{h^{r}} \quad \text { and } \quad\left\|I-J_{h}\right\|_{H^{\sigma} \rightarrow H^{\sigma-r}} \leqslant h^{r} .
$$

One reason it is interesting to assume that $\jmath$ has compact support is the following:

$$
J_{h}=J_{h} J_{c h}, \quad \text { for all } 0 \leqslant c \leqslant 2^{-1} .
$$

3.2. A product estimate. As alluded to above, the Friedrichs mollifiers $J_{h}$ are interesting because they are essentially projection operators (see (3.3)). On the opposite, it is also interesting to use a family of invertible smoothing operators. A good candidate is the family

$$
\left\{\Lambda_{h}^{m}:=\left(I-h^{2} \Delta\right)^{m / 2} \mid m \leqslant 0, h \in(0,1]\right\} .
$$

To begin with, we discuss the properties of these operators which are related to the splitting of the frequency space into two parts: the first where $|\xi| \lesssim$ $1 / h$, the second where $|\xi| \gtrsim 1 / h$.

The operators $\Lambda_{h}^{m}$ depend on the scale parameter $h$. Heuristically, when $m \geqslant 0$, we expect that this $h$-dependence reduces to the following alternative

$$
J_{h} \Lambda_{h}^{m} \lesssim I \quad \text { and } \quad\left(I-J_{h}\right) \Lambda_{h}^{m} \lesssim h^{m}\left|D_{x}\right|^{m} .
$$

As regards the case of negative powers of $\Lambda_{h}$, we will use that

$$
h^{m} \Lambda_{h}^{-m} \lesssim \Lambda^{-m} \text { and } \Lambda_{h}^{-m} \lesssim I .
$$

These statements are made precise by the following lemma.

Lemma 3.1. Let $\left(m_{1}, m_{2}\right) \in \mathbb{R}^{2}$ be such that $0 \leqslant m_{1} \leqslant m_{2}$. Then, for all $h \in[0,1]$ and all $\sigma \in \mathbb{R}$, we have

$$
\left\|h^{m_{1}} \Lambda_{h}^{-m_{2}}\right\|_{H^{\sigma} \rightarrow H^{\sigma+m_{1}}} \leqslant 1 .
$$

For all $m \geqslant 0$ and all $c \in(0,1]$, there exists a positive constant $K$ such that for all $h \in[0,1]$, and all $\sigma \in \mathbb{R}$,

$$
\begin{gathered}
\left\|J_{c h} \Lambda_{h}^{m}\right\|_{H^{\sigma} \rightarrow H^{\sigma}} \leqslant K, \\
\left\|\left(I-J_{c h}\right) \Lambda_{h}^{m}\right\|_{H^{\sigma} \rightarrow H^{\sigma-m}} \leqslant K h^{m} .
\end{gathered}
$$

Proof. To prove these results, we check that, on the symbol level

$$
\begin{gathered}
0 \leqslant h^{m_{1}}\langle h \xi\rangle^{-m_{2}}\langle\xi\rangle^{m_{1}} \leqslant 1, \\
0 \leqslant \jmath(\operatorname{ch} \xi)\langle h \xi\rangle^{-m} \leqslant\left\langle 2 c^{-1}\right\rangle^{m}, \\
0 \leqslant(1-\jmath(\operatorname{ch} \xi))\langle h \xi\rangle^{m}\langle\xi\rangle^{-m} \leqslant h^{m}\langle c\rangle^{2} .
\end{gathered}
$$


We are now prepared to establish the following product estimates.

Proposition 3.2. Let $\sigma_{0}>d / 2,\left(\sigma_{1}, \sigma_{2}\right) \in \mathbb{R}_{+}^{2}$ and $\left(m_{1}, m_{2}\right) \in \mathbb{R}_{+}^{2}$ be such that

$$
\sigma_{1}+\sigma_{2}+m_{1}+m_{2} \leqslant 2 \sigma_{0} .
$$

Then, there exists $K$ depending only on $d, \sigma_{0}, \sigma_{i}, m_{i}$ such that for all $h \in$ $[0,1]$ and $u_{i} \in H^{\sigma_{0}-\sigma_{i}-m_{i}}$,

$$
\left\|\Lambda_{h}^{-m_{1}-m_{2}}\left(u_{1} u_{2}\right)\right\|_{H^{\sigma_{0}-\sigma_{1}-\sigma_{2}}} \leqslant K\left\|\Lambda_{h}^{-m_{1}} u_{1}\right\|_{H^{\sigma_{0}-\sigma_{1}}}\left\|\Lambda_{h}^{-m_{2}} u_{2}\right\|_{H^{\sigma_{0}-\sigma_{2}}} .
$$

This result extends to vector valued functions.

Remark 3.3. In words, this proposition just says that the smoothing effect of the operators $\Lambda_{h}^{-m}$ is distributive.

Proof. The key point is that the operators $\Lambda_{h}^{-m}$ are invertible. It allows us to derive the desired product estimates from the corresponding results in the usual setting $m_{1}=m_{2}=0$. To do so Proposition 3.2 is better formulated as follows: there exists $K$ such that for all $h \in[0,1]$ and $f_{i} \in H^{\sigma_{0}-\sigma_{i}}\left(\mathbb{R}^{d}\right)$,

$$
\left\|\Lambda_{h}^{-m_{1}-m_{2}}\left\{\left(\Lambda_{h}^{m_{1}} f_{1}\right)\left(\Lambda_{h}^{m_{2}} f_{2}\right)\right\}\right\|_{H^{\sigma_{0}-\sigma_{1}-\sigma_{2}}} \leqslant K\left\|f_{1}\right\|_{H^{\sigma_{0}-\sigma_{1}}}\left\|f_{2}\right\|_{H^{\sigma_{0}-\sigma_{2}}} .
$$

The proof of this claim is based on the decomposition of each function $f_{i}$ into two pieces: its low wave number part $J_{h} f_{i}$, and its high wave number part $\left(I-J_{h}\right) f_{i}$. This leads to four products that are handled the same way introducing, for $c \in\{0,1\}$ and $m \in \mathbb{R}$, the Fourier multipliers

$$
\Theta_{c, m}^{h}:=h^{-c m} \Lambda_{h}^{m}\left(J_{h}-c I\right) .
$$

That is, as is easily verified from the triangle inequality, the left-hand side of (3.8) is less than

$$
\sum_{0 \leqslant c_{1}, c_{2} \leqslant 1}\left\|h^{c_{1} m_{1}+c_{2} m_{2}} \Lambda_{h}^{-m_{1}-m_{2}}\left\{\left(\Theta_{c_{1}, m_{1}}^{h} f_{1}\right)\left(\Theta_{c_{2}, m_{2}}^{h} f_{2}\right)\right\}\right\|_{H^{\sigma_{0}-\sigma_{1}-\sigma_{2}}} .
$$

Hence, to prove the claim (3.8) it suffices now to combine three ingredients:

$$
\begin{aligned}
& \left\|h^{c_{1} m_{1}+c_{2} m_{2}} \Lambda_{h}^{-m_{1}-m_{2}} v\right\|_{H^{\sigma_{0}-\sigma_{1}-\sigma_{2}}} \leqslant\|v\|_{H^{\sigma_{0}-\sigma_{1}-\sigma_{2}-c_{1} m_{1}-c_{2} m_{2}}}, \\
& \left\|v_{1} v_{2}\right\|_{H^{\sigma_{0}-\sigma_{1}-\sigma_{2}-c_{1} m_{1}-c_{2} m_{2}}} \lesssim\left\|v_{1}\right\|_{H^{\sigma_{0}-\sigma_{1}-c_{1} m_{1}}}\left\|v_{2}\right\|_{H^{\sigma_{0}-\sigma_{2}-c_{2} m_{2}}}, \\
& \left\|\Theta_{c_{i}, m_{i}}^{h} v\right\|_{H^{\sigma_{0}-\sigma_{i}-c_{i} m_{i}}} \lesssim\|v\|_{H^{\sigma_{0}-\sigma_{i}}} \quad(i \in\{1,2\}) .
\end{aligned}
$$

The first and last inequalities follow from Lemma 3.1. In order to prove the second one, we first recall the classical rule of product in Sobolev spaces (see Theorem 8.3.1. in [17]). For $\left(r_{1}, r_{2}\right) \in \mathbb{R}^{2}$, the product maps continuously $H^{r_{1}}\left(\mathbb{R}^{d}\right) \times H^{r_{2}}\left(\mathbb{R}^{d}\right)$ to $H^{r}\left(\mathbb{R}^{d}\right)$ whenever

$$
r_{1}+r_{2} \geqslant 0, \quad r \leqslant \min \left\{r_{1}, r_{2}\right\} \quad \text { and } \quad r \leqslant r_{1}+r_{2}-d / 2,
$$


with the third inequality strict if $r_{1}$ or $r_{2}$ or $-r$ is equal to $d / 2$. We next verify that (3.9) applies with

$$
r:=\sigma_{0}-\sigma_{1}-\sigma_{2}-c_{1} m_{1}-c_{2} m_{2}, \quad r_{i}:=\sigma_{0}-\sigma_{i}-c_{i} m_{i} \quad \text { for } i=1,2 .
$$

3.3. A Friedrichs' Lemma. In this paragraph we present a result which complements the standard Friedrichs' Lemma. To do that we first need a commutator estimate, stating that the commutator of a Fourier multiplier of order $m$ and the multiplication by a function is an operator of order $m-1$.

Lemma 3.4. Let $\sigma_{0}>d / 2+1$ and $m \in[0,+\infty)$. For any bounded subset $\mathcal{B}$ of $S^{m}$ and all $\sigma \in\left(-\sigma_{0}+m, \sigma_{0}-1\right]$, there exists a constant $K$ such that for all symbol $q \in \mathcal{B}$, all $f \in H^{\sigma_{0}}\left(\mathbb{R}^{d}\right)$, and all $v \in H^{\sigma}\left(\mathbb{R}^{d}\right)$,

$$
\|\mathcal{Q}(f u)-f \mathcal{Q} u\|_{H^{\sigma-m+1}} \leqslant K\|f\|_{H^{\sigma_{0}}}\|u\|_{H^{\sigma}},
$$

where $\mathcal{Q}$ is the Fourier multiplier with symbol $q$.

Lemma 3.4 is classical. Yet, for the convenience of the reader, we include a proof at the end of this paragraph.

A word of caution: the estimate (3.10) carries over to matrix valued functions and symbols except for one key point. Suppose that $f$ and $q$ are matrix valued. In order that (3.10) be true the following condition must be fulfilled: $q(\xi) f(x)=f(x) q(\xi)$ for all $(x, \xi) \in \mathbb{R}^{2 d}$.

Proposition 3.5. Let $\sigma_{0}>d / 2+1$ and $m \in[0,1]$. For all $\sigma$ in the interval $\left(-\sigma_{0}+m, \sigma_{0}-1\right]$, there exists a constant $K$, such that for all $h \in(0,1]$, all $f \in H^{\sigma_{0}}\left(\mathbb{R}^{d}\right)$ and all $u \in H^{-\sigma_{0}}\left(\mathbb{R}^{d}\right)$,

$$
\left\|J_{h}(f u)-f J_{h} u\right\|_{H^{\sigma-m+1}} \leqslant h^{m} K\|f\|_{H^{\sigma_{0}}}\left\|\Lambda_{h}^{-\left(\sigma_{0}+\sigma\right)} u\right\|_{H^{\sigma}} .
$$

Remark 3.6. The thing of interest here is that the precise rate of convergence does not require much on the high wave number part of $u$.

Proof. Given $\mu \in \mathbb{R}$ and $g \in H^{\mu}\left(\mathbb{R}^{d}\right)$, we denote by $g^{b}$ the multiplication operator $H^{-\mu}\left(\mathbb{R}^{d}\right) \ni u \mapsto g u \in \mathcal{S}^{\prime}\left(\mathbb{R}^{d}\right)$. Then, Proposition 3.5 can be formulated concisely in the following way. There exists a constant $K$, depending only on $\sigma_{0}, m$ and $\sigma$, such that for all $h \in(0,1]$,

$$
\left\|\Lambda^{1-m}\left[J_{h}, f^{b}\right] \Lambda_{h}^{\sigma_{0}+\sigma}\right\|_{H^{\sigma} \rightarrow H^{\sigma}} \leqslant h^{m} K\|f\|_{H^{\sigma_{0}}} .
$$

The proof of this claim makes use of the division of the frequency space into two pieces. The low frequencies region $|\xi| \lesssim 1 / h$ and the high frequencies region $|\xi| \gtrsim 1 / h$. We write

$$
\Lambda^{1-m}\left[J_{h}, f^{b}\right] \Lambda_{h}^{\sigma_{0}+\sigma}=C_{0}^{h}+C_{\infty}^{h},
$$

where

$$
\begin{aligned}
C_{0}^{h} & :=\Lambda^{1-m}\left[J_{h}, f^{b}\right] J_{h}^{\prime} \Lambda_{h}^{\sigma_{0}+\sigma}, \\
C_{\infty}^{h} & :=\Lambda^{1-m}\left[J_{h}, f^{b}\right]\left(I-J_{h}^{\prime}\right) \Lambda_{h}^{\sigma_{0}+\sigma},
\end{aligned}
$$


and $J_{h}^{\prime}=J_{5^{-1} h}$. The reason for the particular choice of the constant $5^{-1}$ will be apparent in a moment.

Firstly, we estimate $C_{0}^{h}$. We rewrite $C_{0}^{h}$ as

$$
C_{0}^{h}=h^{m} \Lambda^{1-m}\left[h^{-m}\left(J_{h}-I\right), f^{b}\right] J_{h}^{\prime} \Lambda_{h}^{\sigma_{0}+\sigma} .
$$

The key point to estimate $C_{0}^{h}$ is to notice that $\left\{h^{-m}\left(\jmath_{h}-1\right) \mid 0<h \leqslant 1\right\}$ is a bounded family in $S^{m}$. Indeed, if $q \in S^{m}$ and $q(\xi)$ vanishes for small $\xi$, then the symbols $q_{\lambda}(\cdot)=\lambda^{-m} q(\lambda \cdot)$ belong uniformly to $S^{m}$, for $0<\lambda<\infty$. Once this is granted, Lemma 3.4 implies that the family $\left\{\Lambda^{1-m}\left[h^{-m}\left(J_{h}-I\right), f^{b}\right] \mid 0<h \leqslant 1\right\}$ is bounded in $\mathcal{L}\left(H^{\sigma}\right)$. On the other hand $\left\{J_{h}^{\prime} \Lambda_{h}^{\sigma_{0}+\sigma} \mid 0<h \leqslant 1\right\}$ is a bounded family in $\mathcal{L}\left(H^{\sigma}\right)$, see (3.5). Consequently, in light of (3.12), we end up with

$$
\begin{aligned}
\left\|C_{0}^{h}\right\|_{H^{\sigma} \rightarrow H^{\sigma}} & \leqslant h^{m}\left\|\Lambda^{1-m}\left[h^{-m}\left(J_{h}-I\right), f^{b}\right]\right\|_{H^{\sigma} \rightarrow H^{\sigma}}\left\|J_{h}^{\prime} \Lambda_{h}^{\sigma_{0}+\sigma}\right\|_{H^{\sigma} \rightarrow H^{\sigma}} \\
& \lesssim h^{m}\|f\|_{H^{\sigma_{0}}} .
\end{aligned}
$$

Our next task is to show similar estimates for $C_{\infty}^{h}$. As regards $C_{\infty}^{h}$, the fact that the operators $J_{h}$ are essentially projection operators is the key to the proof. More precisely, we use the identity (3.3) written in the form $J_{h}\left(1-J_{h}^{\prime}\right)=0$ [recall that $J_{h}^{\prime}=J_{5^{-1} h}$ ]. It yields

$$
C_{\infty}^{h}=\Lambda^{1-m} J_{h} f^{b}\left(I-J_{h}^{\prime}\right) \Lambda_{h}^{\sigma_{0}+\sigma} .
$$

It turns out that the situation is even better. Let $v$ belongs to the Schwartz class $\mathcal{S}$. The spectrum (support of the Fourier transform, hereafter denoted by spec $)$ of $\left(J_{h} f\right)\left(\left(I-J_{h}^{\prime}\right) v\right)$ is contained in

$$
\operatorname{spec}\left(J_{h} f\right)+\operatorname{spec}\left(I-J_{h}^{\prime}\right) v \subset B\left(0,2 h^{-1}\right)+B\left(0,10 h^{-1}\right)^{c} \subset B\left(0,3 h^{-1}\right)^{c},
$$

the exterior of the ball centered at 0 of radius $3 h^{-1}$. Which results in

$$
J_{h}\left(\left(J_{h} f\right)\left(\left(I-J_{h}^{\prime}\right) v\right)\right)=0,
$$

that is $\left.J_{h}\left(f\left(\left(I-J_{h}^{\prime}\right) v\right)\right)=J_{h}\left(\left(I-J_{h}\right) f\right)\left(\left(I-J_{h}^{\prime}\right) v\right)\right)$. By combining this identity with (3.13), we are left with

$$
C_{\infty}^{h}=\Lambda^{1-m} J_{h}\left(\left(I-J_{h}\right) f\right)^{b}\left(I-J_{h}^{\prime}\right) \Lambda_{h}^{\sigma_{0}+\sigma} .
$$

To estimate $\left\|C_{\infty}^{h}\right\|_{H^{\sigma} \rightarrow H^{\sigma}}$ we prove a dual estimate for the operator adjoint. We write $\left(C_{\infty}^{h}\right)^{\star}$ as a product of two operators:

$$
\left(C_{\infty}^{h}\right)^{\star}=\left\{h^{-\sigma_{0}-\sigma}\left(I-J_{h}^{\prime}\right) \Lambda_{h}^{\sigma_{0}+\sigma}\right\}\left\{\left(\overline{\left(I-J_{h}\right) f}\right)^{b}\left(h^{\sigma_{0}+\sigma} J_{h}\right) \Lambda^{1-m}\right\},
$$

where $\bar{z}$ denotes the complex conjugated of $z$. The problem reduces to establishing that

$$
\left\|\left(\overline{\left(I-J_{h}\right) f}\right)^{b}\left(h^{\sigma_{0}+\sigma} J_{h} \Lambda^{1-m}\right)\right\|_{H^{-\sigma} \rightarrow H^{\sigma_{0}}} \lesssim h^{m}\|f\|_{H^{\sigma_{0}}} .
$$

Indeed, since the family $\left\{h^{-\sigma_{0}-\sigma}\left(I-J_{h}^{\prime}\right) \Lambda_{h}^{\sigma_{0}+\sigma} \mid 0<h \leqslant 1\right\}$ is bounded in $\mathcal{L}\left(H^{\sigma_{0}} ; H^{-\sigma}\right)$ (see (3.6) $)$, the estimate (3.14) implies that

$$
\left\|\left(C_{\infty}^{h}\right)^{\star}\right\|_{H^{-\sigma} \rightarrow H^{-\sigma}} \lesssim h^{m}\|f\|_{H^{\sigma_{0}}} .
$$


Which in turn implies $\left\|C_{\infty}^{h}\right\|_{H^{\sigma} \rightarrow H^{\sigma}} \lesssim h^{m}\|f\|_{H^{\sigma_{0}}}$ and completes the proof.

We now have to prove (3.14). Let $v$ belongs to the Schwartz class $\mathcal{S}$. Since $\sigma_{0}>d / 2$, we can invoke the standard tame estimate for products, which leads to

$$
\begin{aligned}
\left\|\overline{\left(I-J_{h}\right) f}\left(h^{\sigma_{0}+\sigma} J_{h} \Lambda^{1-m} v\right)\right\|_{H^{\sigma_{0}}} \lesssim \\
\left\|\left(I-J_{h}\right) f\right\|_{L^{\infty}}\left\|\left(h^{\sigma_{0}+\sigma} J_{h} \Lambda^{1-m}\right) v\right\|_{H^{\sigma_{0}}}+ \\
\left\|\left(I-J_{h}\right) f\right\|_{H^{\sigma_{0}}}\left\|\left(h^{\sigma_{0}+\sigma} J_{h} \Lambda^{1-m}\right) v\right\|_{L^{\infty}} .
\end{aligned}
$$

To estimate these fours terms, we use the embedding of $H^{\sigma_{0}-1}\left(\mathbb{R}^{d}\right)$ into $L^{\infty}\left(\mathbb{R}^{d}\right)$ and the bounds given in (3.2), to obtain

$$
\begin{aligned}
\left\|\left(I-J_{h}\right) f\right\|_{L^{\infty}} & \lesssim\left\|\left(I-J_{h}\right) f\right\|_{H^{\sigma_{0}-1}} \lesssim h\|f\|_{H^{\sigma_{0}}}, \\
\left\|\left(h^{\sigma_{0}+\sigma} J_{h} \Lambda^{1-m}\right) v\right\|_{H^{\sigma_{0}}} & =h^{m-1}\left\|\left(h^{\sigma_{0}+\sigma+1-m} J_{h}\right) v\right\|_{H^{\sigma_{0}+1-m}} \lesssim h^{m-1}\|v\|_{H^{-\sigma}}, \\
\left\|\left(I-J_{h}\right) f\right\|_{H^{\sigma_{0}}} & \lesssim\|f\|_{H^{\sigma_{0}}}, \\
\left\|\left(h^{\sigma_{0}+\sigma} J_{h} \Lambda^{1-m}\right) v\right\|_{L^{\infty}} & \lesssim\left\|\left(h^{\sigma_{0}+\sigma} J_{h} \Lambda^{1-m}\right) v\right\|_{H^{\sigma_{0}-1}} \\
& =h^{m}\left\|\left(h^{\sigma_{0}+\sigma-m} J_{h}\right) v\right\|_{H^{\sigma_{0}-m}} \\
& \lesssim h^{m}\|v\|_{H^{\sigma_{0}-m-\left(\sigma_{0}+\sigma-m\right)}}=h^{m}\|v\|_{H^{-\sigma}} .
\end{aligned}
$$

Inserting the four estimates we just proved in (3.15), we conclude that

$$
\left\|\overline{\left(I-J_{h}\right) f}\left(h^{\sigma_{0}+\sigma} J_{h} \Lambda^{1-m} v\right)\right\|_{H^{\sigma_{0}}} \lesssim h^{m}\|f\|_{H^{\sigma_{0}}}\|v\|_{H^{-\sigma}} .
$$

This completes the proof of (3.14).

Proof of Lemma 3.4. To avoid trivialities $(\sigma \in \emptyset)$, assume that $m \leqslant 2 \sigma_{0}-$ 1. We establish the estimate by using the para-differential calculus of Bony [2. In keeping with the notations of the previous proof, $f^{b}$ denotes the multiplication operator $u \mapsto f u$. We denote by $T_{f}$ the operator of para-multiplication by $f$. Rewrite the commutator $\left[\mathcal{Q}, f^{b}\right]$ as

$$
\left[\mathcal{Q}, T_{f}\right]+\mathcal{Q}\left(f^{b}-T_{f}\right)-\left(f^{b}-T_{f}\right) \mathcal{Q} .
$$

The claim then follows from the bounds

$$
\begin{aligned}
& \forall \sigma \in \mathbb{R}, \quad\left\|\left[\mathcal{Q}, T_{f}\right]\right\|_{H^{\sigma} \rightarrow H^{\sigma-m+1}} \leqslant c_{1}(q, \sigma)\|f\|_{H^{\sigma_{0}}}, \\
& \forall \sigma \in\left(-\sigma_{0}, \sigma_{0}-1\right], \quad\left\|f^{b}-T_{f}\right\|_{H^{\sigma} \rightarrow H^{\sigma+1}} \leqslant c_{2}(\sigma)\|f\|_{H^{\sigma_{0}}}, \\
& \forall \sigma \in \mathbb{R}, \quad\|\mathcal{Q}\|_{H^{\sigma} \rightarrow H^{\sigma-m}} \lesssim \sup _{\xi}\left|\langle\xi\rangle^{-m} q(\xi)\right|,
\end{aligned}
$$

where $c_{1}(\cdot, \sigma): S^{m} \rightarrow \mathbb{R}_{+}$takes bounded sets to bounded sets. We refer the reader to [2] and 33] for the proofs of the first two inequalities (see also [17. Prop. 10.2.2] and [17, Th. 9.6.4'] for the proof of (3.16); a detailed proof of (3.17) is given in [17, Prop. 10.2.9]). The estimate (3.18) is obvious. 


\section{ENERGy ESTIMATES FOR THE LINEARIZED SYSTEM}

Many results have been obtained in the past two decades concerning the symmetrization of the Navier Stokes equations (see, e.g., [4, 7, 20, 21]). Yet, the previous works do not include the dimensionless numbers. Here we prove estimates valid for all $a=(\varepsilon, \mu, \kappa)$ in $A$, where $A$ is defined in Notation 1.1. As already written in the introduction, our result improves earlier works [1, 22, 31 on allowing $\kappa \neq 0$. Indeed, when $\kappa=0$, the penalization operator is skew-symmetric and hence the perturbation terms do not appear in the $L^{2}$ estimate, so that the classical proof for solutions to the unperturbed equations holds. In sharp contrast (as observed in 28]), when $\kappa \neq 0$ and the initial temperature variations are large, the problem is more involved because the singular operator is no longer skew-symmetric. Several difficulties also specifically arise for the purpose of proving estimates that are independent of $\mu$ and $\kappa$. In this regard we prove some additional smoothing effects for $\operatorname{div} v$ and $\nabla p$.

We consider the following linearized equations:

$$
\left\{\begin{array}{l}
g_{1}(\phi)\left(\partial_{t} \widetilde{p}+v \cdot \nabla \widetilde{p}\right)+\frac{1}{\varepsilon} \operatorname{div} \widetilde{v}-\frac{\kappa}{\varepsilon} \operatorname{div}\left(\beta_{1}(\phi) \nabla \widetilde{\theta}\right)=f_{1}, \\
g_{2}(\phi)\left(\partial_{t} \widetilde{v}+v \cdot \nabla \widetilde{v}\right)+\frac{1}{\varepsilon} \nabla \widetilde{p}-\mu \beta_{2}(\phi) \Delta \widetilde{v}-\mu \beta_{2}^{\sharp}(\phi) \nabla \operatorname{div} \widetilde{v}=f_{2}, \\
g_{3}(\phi)\left(\partial_{t} \widetilde{\theta}+v \cdot \nabla \widetilde{\theta}\right)+\operatorname{div} \widetilde{v}-\kappa \beta_{3}(\phi) \Delta \widetilde{\theta}=f_{3} .
\end{array}\right.
$$

To fix matters, the unknown $(\widetilde{p}, \widetilde{v}, \widetilde{\theta})$ is a function of the variables $(t, x) \in$ $[0, T] \times \mathbb{D}\left(T\right.$ is a given positive real number and $\mathbb{D}$ denotes either $\mathbb{R}^{d}$ or $\mathbb{T}^{d}$ ) with values in $\mathbb{R} \times \mathbb{R}^{d} \times \mathbb{R}$. The coefficients $\phi=\phi(t, x)$ and $v=v(t, x)$ take their values in $\mathbb{R}^{N}$ and $\mathbb{R}^{d}$, respectively ( $N$ is a given integer).

Parallel to Assumption 2.1, we make the following hypotheses.

Assumption 4.1. Throughout this section we require $g_{1}, g_{2}, g_{3}$ to be $C^{\infty}$ positive functions of $\phi \in \mathbb{R}^{N}$, without recalling this assumption explicitly in the statements. Similarly, it is assumed that $\beta_{1}, \beta_{2}, \beta_{2}^{\sharp}$ and $\beta_{3}$ are $C^{\infty}$ functions of $\phi \in \mathbb{R}^{N}$ satisfying

$$
\beta_{1}>0, \quad \beta_{2}>0, \quad \beta_{2}^{\sharp}+\beta_{2}>0, \quad \beta_{3}>0 .
$$

Our main assumption reads

$$
\beta_{1}<\beta_{3} .
$$

Assumption 4.2. For our purposes, it is sufficient to prove a priori estimates. It is always assumed that the unknown $\widetilde{U}:=(\widetilde{p}, \widetilde{v}, \widetilde{\theta})$, the coefficients $(v, \phi)$ as well as the source term $f:=\left(f_{1}, f_{2}, f_{3}\right)$ are in $C^{0}\left([0, T] ; H^{\infty}(\mathbb{D})\right)$.

4.1. Statements of the results. We establish estimate on $\|\widetilde{U}\|_{\mathcal{H}_{a}^{0}(T)}$ in terms of the norm $\|\widetilde{U}(0)\|_{\mathcal{H}_{a, 0}^{0}}$ of the data and norm of the source term $f$. 
Recall from Definition 2.4 that

$$
\begin{aligned}
& \|\widetilde{U}\|_{\mathcal{H}_{a}^{0}(T)}:=\sup _{t \in[0, T]}\left\{\|(\widetilde{p}, \widetilde{v})(t)\|_{H_{\varepsilon \nu}^{1}}+\|\widetilde{\theta}(t)\|_{H_{\nu}^{1}}\right\} \\
& \quad+\left(\int_{0}^{T} \kappa\|\nabla \widetilde{\theta}\|_{H_{\nu}^{1}}^{2}+\mu\|\nabla \widetilde{v}\|_{H_{\varepsilon \nu}^{1}}^{2}+\kappa\|\operatorname{div} \widetilde{v}\|_{L^{2}}^{2}+(\mu+\kappa)\|\nabla \widetilde{p}\|_{L^{2}}^{2} d t\right)^{\frac{1}{2}},
\end{aligned}
$$

with $\nu:=\sqrt{\mu+\kappa}$. Recall the following notation we use continually in the sequel: given $\sigma \in \mathbb{R}$ and $\varrho \geqslant 0$ we set $\|\cdot\|_{H_{\varrho}^{\sigma}}:=\|\cdot\|_{H^{\sigma-1}}+\varrho\|\cdot\|_{H^{\sigma}}$.

Theorem 4.3. There is a smooth non-decreasing function $C$ from $[0,+\infty)$ to $[0,+\infty)$ such that for all $a \in A$, all $T \in(0,1]$, all coefficients $v$ and $\phi$, and all $(\widetilde{U}, f)$ satisfying (4.1), the norm $\|\widetilde{U}\|_{\mathcal{H}_{a}^{0}(T)}$ satisfies the estimate

$\|\widetilde{U}\|_{\mathcal{H}_{a}^{0}(T)} \leqslant C\left(R_{0}\right) e^{T C(R)}\|\widetilde{U}(0)\|_{\mathcal{H}_{a, 0}^{0}}+C(R) \int_{0}^{T}\left\|\left(f_{1}, f_{2}\right)\right\|_{H_{\varepsilon \nu}^{1}}+\left\|f_{3}\right\|_{H_{\nu}^{1}} d t$,

where $\nu:=\sqrt{\mu+\kappa}$ and

$$
R_{0}:=\|\phi(0)\|_{L^{\infty}(\mathbb{D})}, \quad R:=\sup _{t \in[0, T]}\left\|\left(\phi, \partial_{t} \phi, \nabla \phi, \nu \nabla^{2} \phi, v, \nabla v\right)(t)\right\|_{L^{\infty}(\mathbb{D})} .
$$

Remark 4.4. By Assumption 4.2] all the functions we encounter in the previous statement are in $C^{0}\left([0, T] ; H^{\infty}(\mathbb{D})\right)$. Yet, one can verify that the estimate is valid whenever its two sides are well defined.

Theorem 4.3 is not enough for the purpose of proving high frequency estimates independent of $\kappa$. We will need the following version.

Theorem 4.5. The statement of Theorem 4.3 remains valid if the system (4.1) is replaced with

$$
\left\{\begin{array}{l}
g_{1}(\phi)\left(\partial_{t} \widetilde{p}+v \cdot \nabla \widetilde{p}\right)+\frac{1}{\varepsilon} \operatorname{div} \widetilde{v}-\frac{\kappa}{\varepsilon} \operatorname{div}\left(\beta_{1}(\phi) \nabla \widetilde{\theta}\right)=f_{1}, \\
g_{2}(\phi)\left(\partial_{t} \widetilde{v}+v \cdot \nabla \widetilde{v}\right)+\frac{1}{\varepsilon} \nabla \widetilde{p}-\mu \beta_{2}(\phi) \Delta \widetilde{v}-\mu \beta_{2}^{\sharp}(\phi) \nabla \operatorname{div} \widetilde{v}=f_{2}, \\
g_{3}(\phi)\left(\partial_{t} \widetilde{\theta}+v \cdot \nabla \widetilde{\theta}\right)+G(\phi, \nabla \phi) \cdot \widetilde{v}+\operatorname{div} \widetilde{v}-\kappa \beta_{3}(\phi) \Delta \widetilde{\theta}=f_{3},
\end{array}\right.
$$

where $G$ is smooth in its arguments with values in $\mathbb{R}^{d}$.

The proof of Theorem 4.3 relies upon two $L^{2}$ estimates. Because they may be useful in other circumstances, we give separate statements in 4.3 . The proof of Theorem 4.5 follows from a close inspection of the proof of Theorem 4.3, and so will be omitted.

4.2. Example. Some important features of the proof of Theorem 4.3 can be revealed by analyzing the following simplified system:

$$
\left\{\begin{array}{l}
\partial_{t} p+\frac{1}{\varepsilon} \operatorname{div} v-\frac{1}{\varepsilon} \Delta \theta=0 \\
\partial_{t} v+\frac{1}{\varepsilon} \nabla p=0 \\
\partial_{t} \theta+\operatorname{div} v-\beta \Delta \theta=0 .
\end{array}\right.
$$


For the sake of notational simplicity we abandon the tildes in this subsection. Parallel to (4.2), we suppose

$$
\beta>1 \text {. }
$$

To symmetrize the large terms in $\varepsilon^{-1}$, we introduce $v_{e}:=v-\nabla \theta$. This change of variables transforms (4.5) into

$$
\left\{\begin{array}{l}
\partial_{t} p+\frac{1}{\varepsilon} \operatorname{div} v_{e}=0 \\
\partial_{t} v_{e}+\frac{1}{\varepsilon} \nabla p-\nabla \operatorname{div} v_{e}+(\beta-1) \nabla \Delta \theta=0 \\
\partial_{t} \theta+\operatorname{div} v_{e}-(\beta-1) \Delta \theta=0
\end{array}\right.
$$

We take the $L^{2}$ scalar product of the first [resp. the second] equation with $p$ [resp. $v_{e}$ ]. It yields:

$$
\frac{1}{2} \frac{d}{d t}\left\|\left(p, v_{e}\right)\right\|_{L^{2}}^{2}+\left\|\operatorname{div} v_{e}\right\|_{L^{2}}^{2}-(\beta-1)\left\langle\Delta \theta, \operatorname{div} v_{e}\right\rangle=0 .
$$

We take the $L^{2}$ scalar product of the third equation with $-\eta \Delta \theta$, where $\eta$ is a positive constant to be determined later on. It yields

$$
\begin{aligned}
& \frac{1}{2} \frac{d}{d t}\left\|\left(p, v_{e}, \sqrt{\eta} \nabla \theta\right)\right\|_{L^{2}}^{2} \\
& \quad+\left\|\operatorname{div} v_{e}\right\|_{L^{2}}^{2}-(\beta-1+\eta)\left\langle\Delta \theta, \operatorname{div} v_{e}\right\rangle+\eta(\beta-1)\|\Delta \theta\|_{L^{2}}^{2}=0 .
\end{aligned}
$$

Set $\eta:=\beta-1$, which is positive thanks to assumption (4.6). We get

$$
\frac{1}{2} \frac{d}{d t}\left\|\left(p, v_{e}, \sqrt{\beta-1} \nabla \theta\right)\right\|_{L^{2}}^{2}+\left\|\operatorname{div} v_{e}-(\beta-1) \Delta \theta\right\|_{L^{2}}^{2}=0 .
$$

Let $t \geqslant 0$. Integrating the previous identity from 0 to $t$, and using the triangle inequality to replace $v_{e}$ by $v$, we get

$$
\|(p, v, \nabla \theta)(t)\|_{L^{2}}^{2}+\int_{0}^{t}\|\operatorname{div} v-\beta \Delta \theta\|_{L^{2}}^{2} d \tau \leqslant K_{\beta}\|(p, v, \nabla \theta)(0)\|_{L^{2}}^{2} .
$$

Here and below, $K_{\beta}$ denotes a generic constant which depends only on $\beta$.

We thus have proved an $L^{2}$ estimate independent of $\varepsilon$. To go beyond and obtain smoothing effect on $\operatorname{div} v$ it is sufficient to estimate $\Delta \theta$ independently. The strategy is to incorporate the troublesome term $\operatorname{div} v$ [in the equation for $\theta$ ] into a skew-symmetric operator.

To do so introduce

$$
\zeta:=\varepsilon \beta p-\theta \quad \text { and } \quad v_{\varepsilon}:=\varepsilon v .
$$

We compute

$$
\left\{\begin{array}{l}
\partial_{t} \zeta+\frac{\beta-1}{\varepsilon} \operatorname{div} v_{\varepsilon}=0, \\
\partial_{t} v_{\varepsilon}+\frac{1}{\beta \varepsilon} \nabla \zeta+\frac{1}{\beta \varepsilon} \nabla \theta=0 \\
\partial_{t} \theta+\frac{1}{\varepsilon} \operatorname{div} v_{\varepsilon}-\beta \Delta \theta=0 . \\
19
\end{array}\right.
$$


We now use the essential feature of the system, that is assumption (4.6). Multiply the first equation [resp. the second] by $1 /(\beta-1)$ [resp. $\beta$ ], to put the penalization operator in symmetric form. The energy estimate thus reads

$$
\frac{1}{2} \frac{d}{d t}\left\|\left(\sqrt{1 /(\beta-1)} \zeta, \sqrt{\beta} v_{\varepsilon}, \theta\right)\right\|_{L^{2}}^{2}+\beta\|\nabla \theta\|_{L^{2}}^{2}=0 .
$$

Integrate this inequality, to obtain

$$
\left\|\left(\zeta, v_{\varepsilon}, \theta\right)(t)\right\|_{L^{2}}^{2}+\int_{0}^{t}\|\nabla \theta\|_{L^{2}}^{2} d \tau \leqslant K_{\beta}\left\|\left(\zeta, v_{\varepsilon}, \theta\right)(0)\right\|_{L^{2}}^{2} .
$$

Since the coefficients are constants, the same estimate holds true for the first order derivatives of $\zeta, v_{\varepsilon}$ and $\theta$. Namely, one has

$$
\left\|\nabla\left(\zeta, v_{\varepsilon}, \theta\right)(t)\right\|_{L^{2}}^{2}+\int_{0}^{t}\left\|\nabla^{2} \theta\right\|_{L^{2}}^{2} d \tau \leqslant K_{\beta}\left\|\nabla\left(\zeta, v_{\varepsilon}, \theta\right)(0)\right\|_{L^{2}}^{2} .
$$

On applying the triangle inequality, one can replaced $\zeta$ with $\varepsilon p$ in the previous estimates. Finally, by combining (4.8), (4.9) and (4.10), we get

$$
\begin{gathered}
\|(p, v, \theta)(t)\|_{L^{2}}+\|\nabla(\theta, \varepsilon p, \varepsilon v)(t)\|_{L^{2}}+\left(\int_{0}^{t}\|\operatorname{div} v\|_{L^{2}}^{2}+\|\nabla \theta\|_{H^{1}}^{2} d \tau\right)^{1 / 2} \\
\leqslant K_{\beta}\|(p, v, \theta)(0)\|_{L^{2}}+K_{\beta}\|\nabla(\theta, \varepsilon p, \varepsilon v)(0)\|_{L^{2}} .
\end{gathered}
$$

It is worth remarking that, to establish (4.11), it is enough to prove (4.7) and (4.10). Let us now compare (4.11) with the estimate given in Theorem 4.3. We see that the previous study fails to convey one feature of the proof, namely the usefulness of the additional smoothing effect for $\nabla p$. The reason is that we worked with constant coefficients. In the general case, an estimate for $\int_{0}^{t}\|\nabla p\|_{L^{2}}^{2} d \tau$ is needed to control the left hand side of (4.10) (see Lemma 4.12 below).

Let us explain how to estimate $\int_{0}^{t}\|\nabla p\|_{L^{2}}^{2} d \tau$. Multiply the second equation in (4.5) by $\varepsilon \nabla p$ and integrate over the strip $[0, t] \times \mathbb{D}$, to obtain

$$
\int_{0}^{t}\|\nabla p\|_{L^{2}}^{2} d \tau=-\int_{0}^{t}\left\langle\partial_{t} v, \varepsilon \nabla p\right\rangle d \tau
$$

Integrating by parts both in space and time yields

$$
\begin{aligned}
\int_{0}^{t}\left\langle\varepsilon \partial_{t} v, \nabla p\right\rangle d \tau & =-\int_{0}^{t}\left\langle v, \varepsilon \partial_{t} \nabla p\right\rangle d \tau+\varepsilon[\langle v(\tau), \nabla p(\tau)\rangle]_{\tau=0}^{\tau=t} \\
& =\int_{0}^{t}\left\langle\operatorname{div} v, \varepsilon \partial_{t} p\right\rangle d \tau+\varepsilon[\langle v(\tau), \nabla p(\tau)\rangle]_{\tau=0}^{\tau=t}
\end{aligned}
$$

In view of the first equation in (4.5), we are left with

$$
\int_{0}^{t}\|\nabla p\|_{L^{2}}^{2} d \tau=\int_{0}^{t}\|\operatorname{div} v\|_{L^{2}}^{2}-\langle\operatorname{div} v, \Delta \theta\rangle d \tau-\varepsilon[\langle v(\tau), \nabla p(\tau)\rangle]_{\tau=0}^{\tau=t} .
$$

All the terms that appear in the previous identity have been estimated previously. As a consequence the estimate (4.11) holds true if we include 
$\int_{0}^{t}\|\nabla p\|_{L^{2}}^{2} d \tau$ in its left hand side. By so doing, we obtain the exact analogue of the estimate given in Theorem 4.3 (for $\mu=0$ and $\kappa=1$ ).

4.3. $L^{2}$ estimates. Guided by the previous example, we want to prove $L^{2}$ estimates for $(\widetilde{p}, \widetilde{v})$ and $(\widetilde{\theta}, \varepsilon \widetilde{p}, \varepsilon \widetilde{v})$. The strategy for proving both estimates is the same: transform the system (4.1) so as to obtain $L^{2}$ estimates uniform in $\varepsilon$ by a simple integration by parts in which the large terms in $1 / \varepsilon$ cancel out. Namely, we want to obtain systems having the form

$$
\mathcal{L}_{1}(v, \phi) \mathcal{U}-\mathcal{L}_{2}(\mu, \kappa, \phi) \mathcal{U}+\frac{1}{\varepsilon} S(\phi) \mathcal{U}=F
$$

where $\mathcal{L}_{1}(v, \phi)-\mathcal{L}_{2}(\mu, \kappa, \phi)$ is a mixed hyperbolic/parabolic system of equations:

$$
L_{0}(\phi) \partial_{t}+\sum_{1 \leqslant j \leqslant d} L_{j}(v, \phi) \partial_{j}-\sum_{1 \leqslant j, k \leqslant d} L_{j k}(\mu, \kappa, \phi) \partial_{j} \partial_{k},
$$

and the singular perturbation $S(\phi)$ is a differential operator in the space variable which is skew-symmetric (with not necessarily constant coefficients).

We first prove an estimate parallel to (4.9).

Proposition 4.6. Using the same notations as in Theorem 4.3, we have

$$
\begin{aligned}
& \sup _{t \in[0, T]}\|(\widetilde{\theta}, \varepsilon \widetilde{p}, \varepsilon \widetilde{v})(t)\|_{L^{2}}+\left(\int_{0}^{T} \kappa\|\nabla \widetilde{\theta}\|_{L^{2}}^{2}+\mu\|\varepsilon \nabla \widetilde{v}\|_{L^{2}}^{2} d t\right)^{1 / 2} \\
& \leqslant C\left(R_{0}\right) e^{T C(R)}\|(\widetilde{\theta}, \varepsilon \widetilde{p}, \varepsilon \widetilde{v})(0)\|_{L^{2}}+C(R)\left(\int_{0}^{T} B_{\varepsilon}(f, \widetilde{U}) d t\right)^{1 / 2},
\end{aligned}
$$

where

$$
B_{\varepsilon}(f, \widetilde{U}):=\left\|\left(f_{3}, \varepsilon f_{1}, \varepsilon f_{2}\right)\right\|_{L^{2}}\|(\widetilde{\theta}, \varepsilon \widetilde{p}, \varepsilon \widetilde{v})\|_{L^{2}} .
$$

Corollary 4.7. The estimate (4.13) holds true with $\left(\int_{0}^{T} B_{\varepsilon}(f, \widetilde{U}) d t\right)^{1 / 2}$ replaced by

$$
\int_{0}^{T} \varepsilon\left\|\left(f_{1}, f_{2}\right)\right\|_{L^{2}}+\left\|f_{3}\right\|_{L^{2}} d t
$$

Proof of Corollary 4.7 given Proposition 4.6 . One has, for all $\lambda>0$,

$$
\left(\int_{0}^{T} B_{\varepsilon}(f, \widetilde{U}) d t\right)^{1 / 2} \leqslant \frac{1}{\lambda} \sup _{t \in[0, T]}\|(\widetilde{\theta}, \varepsilon \widetilde{p}, \varepsilon \widetilde{v})\|_{L^{2}}+\lambda \int_{0}^{T}\left\|\left(f_{3}, \varepsilon f_{1}, \varepsilon f_{2}\right)\right\|_{L^{2}} d t .
$$

Using (4.13) we obtain the desired result by taking $\lambda$ large enough.

Notation 4.8. Within the proofs of Proposition 4.6 and 4.10, Rather than writing $C(R)$ and $C\left(R_{0}\right)$ in full, we will use the following abbreviations: $C=C(R)$ and $C_{0}=C\left(R_{0}\right)$. So that $C$ denotes generic constants which depend only on $R:=\left\|\left(\phi, \partial_{t} \phi, \nabla \phi, \nu \nabla^{2} \phi, v, \nabla v\right)\right\|_{L^{\infty}([0, T] \times \mathbb{D})}$ and $C_{0}$ denotes generic constants which depend only on $R_{0}:=\|\phi(0)\|_{L^{\infty}(\mathbb{D})}$. As usual, the values of $C_{0}$ and $C$ may vary from relation to relation. 
Proof of Proposition 4.6. All the computations given below are meaningful since we concentrate on regular solutions of (4.1). Introduce

$$
\widetilde{\zeta}:=\varepsilon g_{1} \beta_{3} \widetilde{p}-g_{3} \beta_{1} \widetilde{\theta}, \quad \widetilde{v}_{\varepsilon}:=\varepsilon \widetilde{v} \quad \text { and } \quad \widetilde{\mathcal{U}}:=\left(\widetilde{\zeta}, \widetilde{v}_{\varepsilon}, \widetilde{\theta}\right)^{t} .
$$

where we simply write $g_{i}, \beta_{i}$ instead of $g_{i}(\phi), \beta_{i}(\phi)$. We first show that $\tilde{\mathcal{U}}$ solves a system of the form (4.12). Expressing $\widetilde{p}$ in terms of $\widetilde{\zeta}$ and $\widetilde{\theta}$, replacing $\widetilde{v}$ by $\varepsilon^{-1} \widetilde{v}_{\varepsilon}$ and performing a little algebra, yields

$$
\begin{aligned}
& \partial_{t} \widetilde{\zeta}+v \cdot \nabla \widetilde{\zeta}+\frac{\beta_{3}-\beta_{1}}{\varepsilon} \operatorname{div} \widetilde{v}_{\varepsilon}=f_{1}^{\prime}, \\
& g_{2}\left(\partial_{t} \widetilde{v}_{\varepsilon}+v \cdot \nabla \widetilde{v}_{\varepsilon}\right)+\frac{1}{\varepsilon} \nabla\left(\gamma_{1} \widetilde{\zeta}\right)+\frac{1}{\varepsilon} \nabla\left(\gamma_{2} \widetilde{\theta}\right)-\mu \beta_{2} \Delta \widetilde{v}_{\varepsilon}-\mu \beta_{2}^{\sharp} \nabla \operatorname{div} \widetilde{v}_{\varepsilon}=\varepsilon f_{2}, \\
& g_{3}\left(\partial_{t} \widetilde{\theta}+v \cdot \nabla \widetilde{\theta}\right)+\frac{1}{\varepsilon} \operatorname{div} \widetilde{v}_{\varepsilon}-\kappa \beta_{3} \Delta \widetilde{\theta}=f_{3}, \\
& \text { where } \gamma_{1}:=\frac{1}{g_{1} \beta_{3}}, \gamma_{2}:=\frac{g_{3} \beta_{1}}{g_{1} \beta_{3}} \text { and } \\
& \qquad \begin{aligned}
f_{1}^{\prime}:= & \varepsilon \beta_{3} f_{1}-\beta_{1} f_{3}+\kappa \beta_{3} \nabla \beta_{1} \cdot \nabla \widetilde{\theta} \\
& +\varepsilon \widetilde{p}\left(\partial_{t}\left(g_{1} \beta_{3}\right)+v \cdot \nabla\left(g_{1} \beta_{3}\right)\right)-\widetilde{\theta}\left(\partial_{t}\left(g_{3} \beta_{1}\right)+v \cdot\left(\nabla g_{3} \beta_{1}\right)\right) .
\end{aligned}
\end{aligned}
$$

In order to symmetrize these equations we make use of the structural assumption (4.2), that is $\beta_{3}-\beta_{1}>0$. Multiply the first equation [resp. the third] by $\gamma_{1}\left(\beta_{3}-\beta_{1}\right)^{-1}$ [resp. $\gamma_{2}$ ]. By so doing, we obtain that $\tilde{\mathcal{U}}$ solves

$$
\mathcal{L}_{1}(v, \phi) \widetilde{\mathcal{U}}-\mathcal{L}_{2}(\mu, \kappa, \phi) \widetilde{\mathcal{U}}+\frac{1}{\varepsilon} S(\phi) \widetilde{\mathcal{U}}=F
$$

with $F:=\left(\gamma_{1}\left(\beta_{3}-\beta_{1}\right)^{-1} f_{1}^{\prime}, \varepsilon f_{2}, \gamma_{2} f_{3}\right)^{T}, \mathcal{L}_{1}(v, \phi):=L_{0}(\phi) \partial_{t}+\sum L_{j}(v, \phi) \partial_{j}$ where

$$
L_{0}:=\left(\begin{array}{ccc}
\frac{\gamma_{1}}{\beta_{3}-\beta_{1}} & 0 & 0 \\
0 & g_{2} I_{d} & 0 \\
0 & 0 & \gamma_{2} g_{3}
\end{array}\right) \quad \text { and } \quad L_{j}:=v_{j} L_{0}
$$

Moreover, the singular and viscous perturbations are defined by

$$
S=\left(\begin{array}{ccc}
0 & \gamma_{1} \operatorname{div} & 0 \\
\nabla\left(\gamma_{1} \cdot\right) & 0 & \nabla\left(\gamma_{2} \cdot\right) \\
0 & \gamma_{2} \operatorname{div} & 0
\end{array}\right), \mathcal{L}_{2}=\left(\begin{array}{ccc}
0 & 0 & 0 \\
0 & \mu \beta_{2} \Delta+\mu \beta_{2}^{\sharp} \nabla \operatorname{div} & 0 \\
0 & 0 & \kappa \gamma_{2} \beta_{3} \Delta
\end{array}\right) .
$$

The end of the proof proceeds by multiplying by $\widetilde{\mathcal{U}}$ and integration by parts. The point is that the large terms in $\varepsilon^{-1}$ cancel out for $S$ is skewsymmetric. Let $t \in(0, T]$. If we multiply (4.15) by $\widetilde{\mathcal{U}}$ and integrate from 0 to $t$, we obtain

$$
\left\langle L_{0}(\phi) \widetilde{\mathcal{U}}, \widetilde{\mathcal{U}}\right\rangle(t)-2 \int_{0}^{t}\left\langle\mathcal{L}_{2}(\phi) \widetilde{\mathcal{U}}, \widetilde{\mathcal{U}}\right\rangle d \tau=\left\langle L_{0}(\phi) \widetilde{\mathcal{U}}, \widetilde{\mathcal{U}}\right\rangle(0)+Y(t)
$$


where $\langle\cdot, \cdot\rangle$ denotes the scalar product in $L^{2}(\mathbb{D})$ and

$$
Y(t):=\int_{0}^{t}\left\langle\left\{\partial_{t} L_{0}(\phi)+\sum_{1 \leqslant j \leqslant d} \partial_{j} L_{j}(v, \phi)\right\} \widetilde{\mathcal{U}}, \widetilde{\mathcal{U}}\right\rangle d \tau+2 \int_{0}^{t}\langle F, \widetilde{\mathcal{U}}\rangle d t .
$$

Here we used the symmetry of the matrices $L_{0}$ and $L_{j}$.

We begin by estimating the right-hand side. One easily gathers

$$
\left\|\partial_{t} L_{0}(\phi)+\sum_{1 \leqslant j \leqslant d} \partial_{j} L_{j}(v, \phi)\right\|_{L^{\infty}} \leqslant C,
$$

(where $C$ is as in Notation 4.8) and

$$
|\langle F, \widetilde{\mathcal{U}}\rangle| \leqslant\|F\|_{L^{2}}\|\widetilde{\mathcal{U}}\|_{L^{2}} \leqslant C\left\|\left(f_{1}^{\prime}, \varepsilon f_{2}, f_{3}\right)\right\|_{L^{2}}\|\widetilde{\mathcal{U}}\|
$$

Furthermore, since by definition $f_{1}^{\prime}$ is a linear combination of $\varepsilon f_{1}, f_{3}, \varepsilon \widetilde{p}$, $\widetilde{\theta}$ and $\kappa \nabla \widetilde{\theta}$ whose coefficients are estimated in $L^{\infty}$ norm by a constant depending only on $R$, we get

$$
\left\|f_{1}^{\prime}\right\| \leqslant C\left\|\left(f_{3}, \varepsilon f_{1}\right)\right\|_{L^{2}}+C\|(\widetilde{\theta}, \varepsilon \widetilde{p})\|_{L^{2}}+C\|\kappa \nabla \widetilde{\theta}\|_{L^{2}} .
$$

Moreover, observe that

$$
(\widetilde{\theta}, \varepsilon \widetilde{p}, \varepsilon \widetilde{v})^{t}=M(\phi) \widetilde{\mathcal{U}},
$$

where $M(\phi)$ is an $n \times n$ invertible matrix. As a consequence, there exists a constant $C$ such that $C^{-1}\|\widetilde{\mathcal{U}}\|_{L^{2}} \leqslant\|(\widetilde{\theta}, \varepsilon \widetilde{p}, \varepsilon v)\|_{L^{2}} \leqslant C\|\widetilde{\mathcal{U}}\|_{L^{2}}$. Hence, the estimates (4.17) and (4.18) result in

$$
|\langle F, \widetilde{\mathcal{U}}\rangle| \leqslant C B_{\varepsilon}(f, \widetilde{U})+C\|\widetilde{\mathcal{U}}\|_{L^{2}}^{2}+C\|\kappa \nabla \widetilde{\theta}\|_{L^{2}}\|\widetilde{\mathcal{U}}\|_{L^{2}},
$$

where $B_{\varepsilon}(f, \widetilde{U})$ is given by (4.14). For all $\lambda \geqslant 1$, the last term in the right hand-side of the previous estimate is bounded by

$$
\lambda C\|\widetilde{\mathcal{U}}\|_{L^{2}}^{2}+\frac{\kappa^{2}}{\lambda}\|\nabla \widetilde{\theta}\|_{L^{2}}^{2} .
$$

Consequently the right-hand side of (4.16) is less than

$$
C_{0}\|\widetilde{\mathcal{U}}(0)\|_{L^{2}}^{2}+\lambda C \int_{0}^{t}\|\widetilde{\mathcal{U}}\|_{L^{2}}^{2} d \tau+\frac{\kappa^{2}}{\lambda} \int_{0}^{t}\|\nabla \widetilde{\theta}\|_{L^{2}}^{2} d \tau+C \int_{0}^{t} B_{\varepsilon}(f, \widetilde{U}) d \tau .
$$

We want to estimate now the left-hand side of (4.16). In this regard, the most direct estimates show that

$$
\left\langle L_{0}(\phi) \widetilde{\mathcal{U}}, \widetilde{\mathcal{U}}\right\rangle \geqslant\left\|L_{0}(\phi)^{-1}\right\|_{L^{\infty}}^{-1}\|\widetilde{\mathcal{U}}\|_{L^{2}}^{2} .
$$

Similarly, using Lemma 4.9 below, one has

$$
-\left\langle\mathcal{L}_{2}(\phi) \widetilde{\mathcal{U}}, \widetilde{\mathcal{U}}\right\rangle \geqslant K \mu m\left\|\nabla \widetilde{v}_{\varepsilon}\right\|^{2}+K \kappa m\|\nabla \widetilde{\theta}\|^{2}-C\|\widetilde{\mathcal{U}}\|_{L^{2}}^{2},
$$

where $m:=\min \left\{\left\|\beta_{2}^{-1}\right\|_{L^{\infty}}^{-1},\left\|\left(\beta_{2}+\beta_{2}^{\sharp}\right)^{-1}\right\|_{L^{\infty}}^{-1},\left\|\beta_{3}^{-1}\right\|_{L^{\infty}}^{-1}\right\}$ and $K$ is a generic constant depending only on the dimension. 
To estimate the $L^{\infty}$ norm of $L_{0}^{-1}, \beta_{2}^{-1},\left(\beta_{2}+\beta_{2}^{\sharp}\right)^{-1}$ and $\beta_{3}^{-1}$, write

$$
\begin{aligned}
\|F(\phi(t))\|_{L^{\infty}} & \leqslant\|F(\phi(0))\|_{L^{\infty}}+\int_{0}^{t}\left\|\partial_{t} F(\phi(\tau))\right\|_{L^{\infty}} d \tau \\
& \leqslant C_{0}+t \sup _{\tau \in[0, t]}\left\|F^{\prime}(\phi(\tau))\right\|_{L^{\infty}}\left\|\partial_{t} \phi(\tau)\right\|_{L^{\infty}} \\
& \leqslant C_{0}+T C \leqslant C_{0} e^{T C} .
\end{aligned}
$$

Consequently, the left hand-side of (4.16) is greater than

$$
C_{0} e^{-T C}\left\{\|\widetilde{\mathcal{U}}(t)\|_{L^{2}}^{2}+\int_{0}^{t} \mu\left\|\nabla \widetilde{v}_{\varepsilon}\right\|^{2}+\kappa\|\nabla \widetilde{\theta}\|^{2} d \tau\right\}-C \int_{0}^{t}\|\widetilde{\mathcal{U}}\|_{L^{2}}^{2} d \tau
$$

From this and the assumption $\kappa \leqslant 1$, we see that one can absorb the third term in (4.21) by taking $\lambda$ large enough. Then, Gronwall's Lemma implies $\|\widetilde{\mathcal{U}}(t)\|_{L^{2}}^{2}+\int_{0}^{t} \mu\left\|\nabla \widetilde{v}_{\varepsilon}\right\|^{2}+\kappa\|\nabla \widetilde{\theta}\|^{2} d \tau \leqslant C_{0} e^{T C}\|\widetilde{\mathcal{U}}(0)\|_{L^{2}}^{2}+C \int_{0}^{t} B_{\varepsilon}(f, \widetilde{U}) d \tau$.

We next claim that the previous estimate holds true with $\tilde{\mathcal{U}}$ replaced by $(\widetilde{\theta}, \varepsilon \widetilde{p}, \varepsilon \widetilde{v})$. Indeed, using the identity (4.19) and the bound (4.22), one has $\|(\widetilde{\theta}, \varepsilon \widetilde{p}, \varepsilon \widetilde{v})\|_{L^{2}} \leqslant C_{0} e^{T C}\|\widetilde{\mathcal{U}}\|_{L^{2}}$. To complete the proof, take the square root of the inequality thus obtained and take the supremum over $t \in[0, T]$.

Let us briefly recall a standard estimate used in the previous proof.

Lemma 4.9. There exists two constants $K_{i}=K_{i}(d)$ such that for all Lipschitz functions $\zeta=\zeta(x)$ and $\eta=\eta(x)$ such that $\zeta>0$ and $\eta+\zeta>0$, and for all vector field $u \in H^{1}(\mathbb{D})$, there holds

$$
-\langle\zeta \Delta u+\eta \nabla \operatorname{div} u, u\rangle_{H^{-1} \times H^{1}} \geqslant K_{1} m\|\nabla u\|_{L^{2}}^{2}-\frac{K_{2} M^{2}}{m}\|u\|_{L^{2}}^{2},
$$

where $m=\inf _{x \in \mathbb{D}}\{\zeta(x), \zeta(x)+\eta(x)\}$ and $M=\|\nabla \zeta\|_{L^{\infty}}+\|\nabla \eta\|_{L^{\infty}}$.

Proof. Decompose $u$ as $\underline{u}+\nabla \phi:=\mathcal{Q} u+(I-\mathcal{Q}) u$ where $\mathcal{Q}$ is the Leray projector onto divergence free vector field ${ }^{2}$, so that

$$
\zeta \Delta u+\eta \nabla \operatorname{div} u=\zeta \Delta \underline{u}+(\zeta+\eta) \nabla \Delta \phi .
$$

It results from $\operatorname{div} \underline{u}=0$ that

$$
-\langle\zeta \Delta u+\eta \nabla \operatorname{div} u, u\rangle_{H^{-1} \times H^{1}}=\langle\zeta \nabla \underline{u}, \nabla \underline{u}\rangle+\langle(\zeta+\eta) \Delta \phi, \Delta \phi\rangle+R,
$$

where $R$ is such that $|R| \leqslant K_{3} M\|\nabla u\|_{L^{2}}\|u\|_{L^{2}}$.

To infer the desired bound, we use two usual inequalities. For all $\lambda \geqslant 1$, one has $|R| \leqslant(m / \lambda)\|\nabla u\|_{L^{2}}^{2}+\left(\lambda K_{3}^{2} M^{2} / m\right)\|u\|_{L^{2}}^{2}$. We next recall the most simplest of all Calderón-Zygmund estimates: $\left\|\nabla^{2} \phi\right\|_{L^{2}} \leqslant K_{4}\|\Delta \phi\|_{L^{2}}$.

We next prove an estimate parallel to (4.7).

\footnotetext{
${ }^{2}$ This trick is due to R. Danchin.
} 
Proposition 4.10. Using the same notations as in Theorem 4.3, we have

$$
\begin{aligned}
\sup _{t \in[0, T]} & \|(\widetilde{p}, \widetilde{v})(t)\|_{L^{2}}+\left(\int_{0}^{T} \mu\|\nabla \widetilde{v}\|_{L^{2}}^{2}+\kappa\|\operatorname{div} \widetilde{v}\|_{L^{2}}^{2} d t\right)^{1 / 2} \\
\leqslant & C\left(R_{0}\right) e^{T C(R)}\|(\widetilde{p}, \widetilde{v})(0)\|_{L^{2}}+C(R) \int_{0}^{T}\left\|\left(f_{1}, f_{2}\right)\right\|_{L^{2}}+\kappa\left\|f_{3}\right\|_{H^{1}} d t \\
& +C\left(R_{0}\right) e^{T C(R)}\left\{\sup _{t \in[0, T]}\|\widetilde{\theta}(t)\|_{H_{\nu}^{1}}+\left(\int_{0}^{T} \kappa\|\nabla \widetilde{\theta}\|_{H_{\nu}^{1}}^{2} d t\right)^{1 / 2}\right\}
\end{aligned}
$$

where $\nu:=\sqrt{\mu+\kappa}$ and $\|\cdot\|_{H_{\nu}^{1}}=\|\cdot\|_{L^{2}}+\nu\|\cdot\|_{H^{1}}$.

Remark 4.11. From now on, we make intensive and implicit uses of the assumptions $\mu \leqslant 1$ and $\kappa \leqslant 1$. In particular we freely use obvious estimates like $\mu \leqslant \sqrt{\mu} \leqslant \nu$. Similarly, we use the estimate $\nu\|\nabla u\|_{L^{2}} \leqslant\|u\|_{H_{\nu}^{1}}$ without mentioning it explicitly in the proofs.

Proof. Introduce $\widetilde{v}_{e}:=\widetilde{v}-\kappa \beta_{1} \nabla \widetilde{\theta}$. Performing a little algebra we find that $\widetilde{\mathcal{V}}:=\left(\widetilde{p}, \widetilde{v}_{e}\right)^{t}$ satisfies

$$
\left(\begin{array}{cc}
g_{1} & 0 \\
0 & g_{2} I_{d}
\end{array}\right)\left(\partial_{t} \tilde{\mathcal{V}}+v \cdot \nabla \widetilde{\mathcal{V}}\right)+\frac{1}{\varepsilon}\left(\begin{array}{cc}
0 & \operatorname{div} \\
\nabla & 0
\end{array}\right) \tilde{\mathcal{V}}-\left(\begin{array}{cc}
0 & 0 \\
0 & \mathcal{L}_{22}
\end{array}\right) \tilde{\mathcal{V}}=F
$$

where

$$
\mathcal{L}_{22}:=\kappa g_{2} \beta_{1} \nabla\left(g_{3}^{-1} \operatorname{div} \cdot\right)+\mu \beta_{2} \Delta+\mu \beta_{2}^{\sharp} \nabla \operatorname{div},
$$

and $F=\left(f_{1}, f_{2}+f_{2}^{\prime}\right)^{t}$ with

$$
\begin{aligned}
f_{2}^{\prime}:= & \mu \kappa \beta_{2} \Delta\left(\beta_{1} \nabla \widetilde{\theta}\right)+\mu \kappa \beta_{2}^{\sharp} \nabla \operatorname{div}\left(\beta_{1} \nabla \widetilde{\theta}\right)-\kappa g_{2}\left(\partial_{t} \beta_{1}+v \cdot \nabla \beta_{1}\right) \nabla \widetilde{\theta} \\
& +\kappa g_{2} \beta_{1} \nabla\left(\kappa g_{3}^{-1} \operatorname{div}\left(\beta_{1} \nabla \widetilde{\theta}\right)-\kappa g_{3}^{-1} \beta_{3} \Delta \widetilde{\theta}-g_{3}^{-1} f_{3}\right)+\kappa g_{2} \beta_{1}(\nabla v)^{t} \nabla \widetilde{\theta} .
\end{aligned}
$$

The thing of greatest interest here is that $-\mathcal{L}_{22}$ is a differential operator whose leading symbol is greater than $C \kappa \xi^{t} \cdot \xi+C \mu|\xi|^{2}$.

As before, the proof proceeds by multiplying by $\widetilde{\mathcal{V}}$ and integrating on the strip $[0, t] \times \mathbb{D}$. Then the analysis establishing that the right [resp. left] hand side of (4.16) is smaller than (4.21) [resp. greater than (4.23)] also gives:

$$
\begin{aligned}
& \|\widetilde{\mathcal{V}}(t)\|_{L^{2}}^{2}+\int_{0}^{t} \mu\left\|\nabla \widetilde{v}_{e}\right\|_{L^{2}}^{2}+\kappa\left\|\operatorname{div} \widetilde{v}_{e}\right\|_{L^{2}}^{2} d \tau \\
& \quad \leqslant C_{0} e^{T C}\|\widetilde{\mathcal{V}}(0)\|_{L^{2}}^{2}+C \int_{0}^{t}\|\widetilde{\mathcal{V}}\|_{L^{2}}^{2} d \tau+C \int_{0}^{t}|\langle F, \widetilde{\mathcal{V}}\rangle| d \tau .
\end{aligned}
$$

Let us estimate the last term in the right-hand side of (4.26). Using (4.25) one can decompose $f_{2}^{\prime}$ as $f_{2,1}^{\prime}+\sqrt{\kappa} \nabla f_{2,2}^{\prime}$ where

$$
\begin{aligned}
& \left\|f_{2,1}^{\prime}\right\|_{L^{2}} \leqslant C \kappa\left\|f_{3}\right\|_{H^{1}}+C \kappa\|\nabla \widetilde{\theta}\|_{L^{2}}+C \sqrt{\kappa}(\mu+\kappa)\left\|\nabla^{2} \widetilde{\theta}\right\|_{L^{2}}, \\
& \left\|f_{2,2}^{\prime}\right\|_{L^{2}} \leqslant C \kappa\|\nabla \widetilde{\theta}\|_{L^{2}}+C \sqrt{\kappa}(\mu+\kappa)\left\|\nabla^{2} \widetilde{\theta}\right\|_{L^{2}} .
\end{aligned}
$$


By notation, $\langle F, \widetilde{\mathcal{V}}\rangle=\left\langle f_{1}, \widetilde{p}\right\rangle+\left\langle f_{2}, \widetilde{v}_{e}\right\rangle+\left\langle f_{2,1}^{\prime}, \widetilde{v}_{e}\right\rangle-\left\langle f_{2,2}^{\prime}, \sqrt{\kappa} \operatorname{div} \widetilde{v}_{e}\right\rangle$. We thus get, for all $\lambda \geqslant 1$,

$$
\begin{aligned}
\int_{0}^{t}|\langle F, \widetilde{\mathcal{V}}\rangle| d \tau \leqslant & \lambda C\left(\int_{0}^{t}\left\|\left(f_{1}, f_{2}\right)\right\|_{L^{2}}+\kappa\left\|f_{3}\right\|_{H^{1}} d \tau\right)^{2} \\
& +\frac{1}{\lambda} \int_{0}^{t} \kappa\left\|\operatorname{div} \widetilde{v}_{e}\right\|_{L^{2}}^{2} d \tau+\frac{1}{\lambda} \sup _{\tau \in[0, t]}\|\widetilde{\mathcal{V}}(t)\|_{L^{2}}^{2} \\
& +\lambda C \int_{0}^{t} \|\left(\kappa \nabla \widetilde{\theta}, \sqrt{\kappa}(\mu+\kappa) \nabla^{2} \widetilde{\theta} \|_{L^{2}}^{2} d \tau\right.
\end{aligned}
$$

Replacing $\widetilde{v}_{e}$ with $\widetilde{v}$ in (4.26)-(4.27), taking $\lambda$ large enough and applying Gronwall's lemma leads to the expected result.

4.4. End of the proof of Theorem 4.3. From now on, we consider a time $0<T \leqslant 1$, a fixed triple of parameter $a=(\varepsilon, \mu, \kappa) \in A$ and a solution $\widetilde{U}=(\widetilde{p}, \widetilde{v}, \widetilde{\theta})$ of the system (4.1). We denote by $R_{0}$ and $R$ the norms defined in the statement of Theorem 4.3 (see (4.3)). We also set $\nu:=\sqrt{\mu+\kappa}$.

Introduce the functions $N, w, y, Y, Z:[0, T] \rightarrow \mathbb{R}_{+}$given by

$$
N(t):=\|\widetilde{U}\|_{\mathcal{H}_{a}^{0}(t)},
$$

and

$$
\begin{gathered}
w(t):=\sup _{\tau \in[0, t]}\|(\widetilde{p}, \widetilde{v})(\tau)\|_{L^{2}}+\left(\int_{0}^{t} \mu\|\nabla \widetilde{v}\|_{L^{2}}^{2}+\kappa\|\operatorname{div} \widetilde{v}\|_{L^{2}}^{2} d \tau\right)^{1 / 2}, \\
y(t):=\sup _{\tau \in[0, t]}\|(\widetilde{\theta}, \varepsilon \widetilde{p}, \varepsilon \widetilde{v})(\tau)\|_{L^{2}}+\left(\int_{0}^{t} \kappa\|\nabla \widetilde{\theta}\|_{L^{2}}^{2}+\mu\|\varepsilon \nabla \widetilde{v}\|_{L^{2}}^{2} d \tau\right)^{1 / 2}, \\
Y(t):=\sup _{\tau \in[0, t]}\|\nabla(\widetilde{\theta}, \varepsilon \widetilde{p}, \varepsilon \widetilde{v})(\tau)\|_{L^{2}}+\left(\int_{0}^{t} \kappa\left\|\nabla^{2} \widetilde{\theta}\right\|_{L^{2}}^{2}+\mu\left\|\varepsilon \nabla^{2} \widetilde{v}\right\|_{L^{2}}^{2} d \tau\right)^{1 / 2}, \\
z(t):=\left(\int_{0}^{t}\|\nabla \widetilde{p}\|_{L^{2}} d \tau\right)^{1 / 2} .
\end{gathered}
$$

Note that $N(T) \leqslant w(T)+\nu z(T)+y(T)+\nu Y(T)$. Since we have estimated $w(T)$ and $y(T)$, it remains only to estimate $\nu Y(T)$ and $\nu z(T)$. Parallel to (4.10), we begin by establishing an estimate for $Y$.

Lemma 4.12. There exists a constant $C_{0}$ depending only on $R_{0}$ and a constant $C$ depending only on $R$ such that for all $t \in[0, T]$ and all $\lambda \geqslant 1$,

$$
\begin{aligned}
Y(t)^{2} \leqslant & C_{0} e^{T C} Y(0)^{2}+\lambda C \int_{0}^{t} Y(\tau)^{2} d \tau+\frac{C}{\lambda} \int_{0}^{t}\|(\operatorname{div} \widetilde{v}, \nabla \widetilde{p})\|_{L^{2}}^{2} d \tau \\
& +\left(C \int_{0}^{t}\left\|f_{3}\right\|_{H^{1}}+\left\|\left(f_{1}, f_{2}\right)\right\|_{H_{\varepsilon}^{1}} d \tau\right)^{2}
\end{aligned}
$$


Proof. The proof, if tedious, is elementary. Indeed, the strategy for proving (4.29) consists in differentiating the system (4.1) so as to apply Proposition 4.6 with $(\widetilde{\theta}, \varepsilon \widetilde{p}, \varepsilon \widetilde{v})$ replaced by $\nabla(\widetilde{\theta}, \varepsilon \widetilde{p}, \varepsilon \widetilde{v})$.

Let $1 \leqslant j \leqslant d$ and set

$$
\widetilde{U}_{j}:=\left(\widetilde{p}_{j}, \widetilde{v}_{j}, \widetilde{\theta}_{j}\right):=\left(\partial_{j} \widetilde{p}, \partial_{j} \widetilde{v}, \partial_{j} \widetilde{\theta}\right) .
$$

We commute ${ }^{3}$ the $i$ th equation in (4.1) with $g_{i}\left(\partial_{j} g_{i}^{-1} \cdot\right)$. It yields

$$
\left\{\begin{array}{l}
g_{1}(\phi)\left(\partial_{t} \widetilde{p}_{j}+v \cdot \nabla \widetilde{p}_{j}\right)+\frac{1}{\varepsilon} \operatorname{div} \widetilde{v}_{j}-\frac{\kappa}{\varepsilon} \operatorname{div}\left(\beta_{1}(\phi) \nabla \widetilde{\theta}_{j}\right)=F_{1}, \\
g_{2}(\phi)\left(\partial_{t} \widetilde{v}_{j}+v \cdot \nabla \widetilde{v}_{j}\right)+\frac{1}{\varepsilon} \nabla \widetilde{p}_{j}-\mu \beta_{2}(\phi) \Delta \widetilde{v}_{j}-\mu \beta_{2}^{\sharp}(\phi) \nabla \operatorname{div} \widetilde{v}_{j}=F_{2}, \\
g_{3}(\phi)\left(\partial_{t} \widetilde{\theta}_{j}+v \cdot \nabla \widetilde{\theta}_{j}\right)+\operatorname{div} \widetilde{v}_{j}-\kappa \beta_{3}(\phi) \Delta \widetilde{\theta}_{j}=F_{3},
\end{array}\right.
$$

where, for $i=1,2,3$, the source term $F_{i}$ is given by

$$
F_{i}:=F_{i}^{1}+F_{i}^{2}:=g_{i} \partial_{j}\left(g_{i}^{-1} f_{i}\right)+g_{i} \tilde{F}_{i}^{2},
$$

with

$$
\begin{aligned}
\tilde{F}_{1}^{2} & :=-\partial_{j} v \cdot \nabla \widetilde{p}-\frac{1}{\varepsilon} \partial_{j} g_{1}^{-1} \operatorname{div} \widetilde{v}+\frac{\kappa}{\varepsilon} \partial_{j} g_{1}^{-1} \operatorname{div}\left(\beta_{1} \nabla \widetilde{\theta}\right)+\frac{\kappa}{\varepsilon} g_{1}^{-1} \operatorname{div}\left(\partial_{j} \beta_{1} \nabla \widetilde{\theta}\right), \\
\tilde{F}_{2}^{2} & :=-\partial_{j} v \cdot \nabla \widetilde{v}-\frac{1}{\varepsilon} \partial_{j} g_{2}^{-1} \nabla \widetilde{p}+\mu \partial_{j}\left(g_{2}^{-1} \beta_{2}\right) \Delta \widetilde{v}+\mu \partial_{j}\left(g_{2}^{-1} \beta_{2}^{\sharp}\right) \nabla \operatorname{div} \widetilde{v}, \\
\tilde{F}_{3}^{2} & :=-\partial_{j} v \cdot \nabla \widetilde{\theta}-\partial_{j} g_{3}^{-1} \operatorname{div} \widetilde{v}+\kappa \partial_{j}\left(g_{3}^{-1} \beta_{3}\right) \Delta \widetilde{\theta} .
\end{aligned}
$$

Proposition 4.6 implies that

$$
Y(t)^{2} \leqslant C_{0} e^{T C}\|\widetilde{\mathcal{U}}(0)\|_{L^{2}}^{2}+C \sum_{1 \leqslant j \leqslant d} \int_{0}^{t} B_{\varepsilon}\left(F, \widetilde{U}_{j}\right) d \tau .
$$

where $\widetilde{\mathcal{U}}:=(\nabla \widetilde{\theta}, \varepsilon \nabla \widetilde{p}, \varepsilon \nabla \widetilde{v})$ and $B_{\varepsilon}$ is defined by (4.14). We now have to estimate $B_{\varepsilon}\left(F, \widetilde{U}_{j}\right)$. The source terms are directly estimated by

$$
\begin{aligned}
& \left\|\left(F_{3}^{1}, \varepsilon F_{2}^{1}, \varepsilon F_{3}^{1}\right)\right\|_{L^{2}} \leqslant C\left\|f_{3}\right\|_{H^{1}}+C\left\|\left(f_{1}, f_{2}\right)\right\|_{H_{\varepsilon}^{1}}, \\
& \left\|\left(\tilde{F}_{3}^{2}, \varepsilon \tilde{F}_{2}^{2}, \varepsilon \tilde{F}_{3}^{2}\right)\right\|_{L^{2}} \leqslant C\|\widetilde{\mathcal{U}}\|_{L^{2}}+C\|(\operatorname{div} \widetilde{v}, \nabla \widetilde{p})\|_{L^{2}}+C\left\|\left(\varepsilon \mu \nabla^{2} \widetilde{v}, \kappa \nabla^{2} \widetilde{\theta}\right)\right\|_{L^{2}} .
\end{aligned}
$$

The last estimate implies that, for all positive $\lambda$ and $\lambda^{\prime}$, one has

$$
\begin{aligned}
B_{\varepsilon}\left(F^{2}, \widetilde{U}_{j}\right) \leqslant & \left(1+\lambda+\lambda^{\prime}\right) C\|\widetilde{\mathcal{U}}\|_{L^{2}}^{2} \\
& +\frac{C}{\lambda}\|(\operatorname{div} \widetilde{v}, \nabla \widetilde{p})\|_{L^{2}}^{2}+\frac{C}{\lambda^{\prime}}\left\|\left(\varepsilon \mu \nabla^{2} \widetilde{v}, \kappa \nabla^{2} \widetilde{\theta}\right)\right\|_{L^{2}}^{2} .
\end{aligned}
$$

Moreover, the term $\int_{0}^{t}\left|B_{\varepsilon}\left(F^{1}, \widetilde{U}_{j}\right)\right| d \tau$ is estimated as in the proof of Corollary 4.7. With these estimates in hands, we find that the last term in the

\footnotetext{
${ }^{3}$ Which means that we commute $\partial_{j}$ with the $i$ th equation $(i=1,2,3)$ premultiplied by $g_{i}^{-1}$ and we next multiply the result by $g_{i}$.
} 
right-side of (4.30) is less then

$$
\begin{aligned}
& \int_{0}^{t}\left(1+\lambda+\lambda^{\prime}\right) C\|\widetilde{\mathcal{U}}\|_{L^{2}}^{2} d \tau+\frac{C}{\lambda^{\prime \prime}} \sup _{\tau \in[0, t]}\|\widetilde{\mathcal{U}}\|_{L^{2}}^{2} \\
& +\int_{0}^{t} \frac{C}{\lambda}\|(\operatorname{div} \widetilde{v}, \nabla \widetilde{p})\|_{L^{2}}^{2} d \tau+\frac{C}{\lambda^{\prime}} \int_{0}^{t}\left\|\left(\varepsilon \mu \nabla^{2} \widetilde{v}, \kappa \nabla^{2} \widetilde{\theta}\right)\right\|_{L^{2}}^{2} d \tau \\
& +\lambda^{\prime \prime}\left(C \int_{0}^{t}\left\|f_{3}\right\|_{H^{1}}+\left\|\left(f_{1}, f_{2}\right)\right\|_{H_{\varepsilon}^{1}} d \tau\right)^{2} .
\end{aligned}
$$

Yet, by definition, $\sup _{\tau \in[0, t]}\|\widetilde{\mathcal{U}}\|_{L^{2}}^{2}+\int_{0}^{t}\left\|\left(\varepsilon \mu \nabla^{2} \widetilde{v}, \kappa \nabla^{2} \widetilde{\theta}\right)\right\|_{L^{2}}^{2} d \tau \leqslant Y(t)^{2}$. Hence, taking $\lambda^{\prime}=\lambda^{\prime \prime}$ large enough, one can absorb the second and fourth terms in (4.31) in the left hand side of (4.30), thereby obtaining the desired estimate (4.29).

To obtain a closed set of inequalities it remains to estimate $z(t)$.

Lemma 4.13. There exists a constant $C_{0}$ depending only on $R_{0}$ and a constant $C$ depending only on $R$ such that for all $t \in[0, T]$,

$$
\nu^{2} z(t)^{2} \leqslant C_{0} e^{T C}\left\{\nu^{2} Y(t)^{2}+w(t)^{2}\right\}+C\left(\int_{0}^{T}\left\|\left(f_{1}, f_{2}\right)\right\|_{L^{2}} d t\right)^{2} .
$$

Proof. Set $\Omega_{t}:=[0, t] \times \mathbb{D}$ and denote by $\left\langle\langle\right.$,$\rangle the scalar product in L^{2}\left(\Omega_{t}\right)$. Multiplying the second equation in (4.1) by $\varepsilon \nu^{2} g_{2}^{-1} \nabla \widetilde{p}$, and integrating over $\Omega_{t}$ yields

$$
\begin{aligned}
\nu^{2}\left\langle\left\langle g_{2}^{-1} \nabla \widetilde{p}, \nabla \widetilde{p}\right\rangle\right\rangle= & \\
& -\varepsilon \nu^{2}\left\langle\left\langle\left(\partial_{t}+v \cdot \nabla\right) \widetilde{v}, \nabla \widetilde{p}\right\rangle\right\rangle+\varepsilon \mu \nu^{2}\left\langle\left\langle g_{2}^{-1} \beta_{2} \Delta \widetilde{v}, \nabla \widetilde{p}\right\rangle\right\rangle \\
& +\varepsilon \mu \nu^{2}\left\langle\left\langle g_{2}^{-1} \beta_{2}^{\sharp} \nabla \operatorname{div} \widetilde{v}, \nabla \widetilde{p}\right\rangle\right\rangle+\varepsilon \nu^{2}\left\langle\left\langle g_{2}^{-1} f_{2}, \nabla \widetilde{p}\right\rangle\right\rangle .
\end{aligned}
$$

The most direct estimates show that

$$
\begin{aligned}
\left\langle\left\langle g_{2}^{-1} \nabla \widetilde{p}, \nabla \widetilde{p}\right\rangle\right\rangle & \geqslant\left\|g_{2}\right\|_{L^{\infty}\left(\Omega_{t}\right)}^{-1}\|\nabla \widetilde{p}\|_{L^{2}\left(\Omega_{t}\right)}^{2}, \\
\varepsilon \mu \nu^{2}\left\langle\left\langle g_{2}^{-1} \beta_{2} \Delta \widetilde{v}, \nabla \widetilde{p}\right\rangle\right\rangle & \leqslant\left\|g_{2}^{-1} \beta_{2}\right\|_{L^{\infty}\left(\Omega_{t}\right)}\left\|\varepsilon \mu \nu \nabla^{2} \widetilde{v}\right\|_{L^{2}\left(\Omega_{t}\right)}\|\nu \nabla \widetilde{p}\|_{L^{2}\left(\Omega_{t}\right)}, \\
\varepsilon \mu \nu^{2}\left\langle\left\langle g_{2}^{-1} \beta_{2}^{\sharp} \nabla \operatorname{div} \widetilde{v}, \nabla \widetilde{p}\right\rangle\right\rangle & \leqslant\left\|g_{2}^{-1} \beta_{2}^{\sharp}\right\|_{L^{\infty}\left(\Omega_{t}\right)}\left\|\varepsilon \mu \nu \nabla^{2} \widetilde{v}\right\|_{L^{2}\left(\Omega_{t}\right)}\|\nu \nabla \widetilde{p}\|_{L^{2}\left(\Omega_{t}\right)}, \\
\varepsilon \nu^{2}\left\langle\left\langle g_{2}^{-1} f_{2}, \nabla \widetilde{p}\right\rangle\right\rangle & \leqslant\left\|g_{2}^{-1}\right\|_{L^{\infty}\left(\Omega_{t}\right)}\left\|f_{2}\right\|_{L_{t}^{1} L^{2}}\left\|\varepsilon \nu^{2} \nabla \widetilde{p}\right\|_{C_{t}^{0} L^{2}},
\end{aligned}
$$

where we used the shorthand notations

$$
\|\cdot\|_{C_{t}^{0} X}:=\sup _{\tau \in[0, t]}\|\cdot\|_{X} \text { and }\|\cdot\|_{L_{t}^{p} X}:=\left(\int_{0}^{t}\|\cdot\|_{X}^{p} d \tau\right)^{1 / p} .
$$

With the bound (4.22), the previous four estimates and (4.33) imply

$$
\begin{aligned}
\|\nu \nabla \widetilde{p}\|_{L^{2}\left(\Omega_{t}\right)}^{2} \leqslant & C_{0} e^{T C}\left|\varepsilon \nu^{2}\left\langle\left\langle\left(\partial_{t}+v \cdot \nabla\right) \widetilde{v}, \nabla \widetilde{p}\right\rangle\right\rangle\right| \\
& +C_{0} e^{T C}\left(\left\|\varepsilon \mu \nu \nabla^{2} \widetilde{v}\right\|_{L^{2}\left(\Omega_{t}\right)}+\left\|f_{2}\right\|_{L_{t}^{1} L^{2}}+\left\|\varepsilon \nu^{2} \nabla \widetilde{p}\right\|_{C_{t}^{0} L^{2}}\right)^{2} .
\end{aligned}
$$


Note that the second term in the right-hand side of the previous estimate is bounded by the right-hand side of (4.32). It thus remains to estimate the first term. To do so integrate by parts in both the space and time variables to obtain

$$
\begin{aligned}
\varepsilon \nu^{2}\left\langle\left\langle\left(\partial_{t}+v \cdot \nabla\right) \widetilde{v}, \nabla \widetilde{p}\right\rangle\right\rangle \\
=\varepsilon \nu^{2}\left\langle\left\langle\operatorname{div} \widetilde{v},\left(\partial_{t}+v \cdot \nabla\right) \widetilde{p}\right\rangle\right\rangle \\
\quad-\varepsilon \nu^{2}\langle\operatorname{div} \widetilde{v}(t), \widetilde{p}(t)\rangle+\varepsilon \nu^{2}\langle\operatorname{div} \widetilde{v}(0), \widetilde{p}(0)\rangle \\
\quad+\varepsilon \nu^{2}\langle\langle\nabla v, \nabla \widetilde{p} \otimes \widetilde{v}\rangle\rangle-\varepsilon \nu^{2}\langle\langle\operatorname{div} v, \widetilde{v} \cdot \nabla \widetilde{p}\rangle\rangle .
\end{aligned}
$$

The last two terms in the right-hand side of (4.35) are estimated by

$$
K t\|\nabla v\|_{C_{t}^{0} L^{\infty}}^{2}\|\widetilde{v}\|_{C_{t}^{0} L^{2}}^{2}+\|\varepsilon \nu \nabla \widetilde{p}\|_{C_{t}^{0} L^{2}}^{2} \leqslant T C w(t)^{2}+\nu^{2} Y(t)^{2},
$$

and the second and third terms are estimated by

$$
\|\varepsilon \nu \nabla \widetilde{v}\|_{C_{t}^{0} L^{2}}^{2}+\|\widetilde{p}\|_{C_{t}^{0} L^{2}}^{2} \leqslant \nu^{2} Y(t)^{2}+w(t)^{2} .
$$

In particular, the sum of the last four terms in the right-hand side of 4.35) is estimated by $e^{T C}\left\{w(t)^{2}+\nu^{2} Y(t)^{2}\right\}$. This brings us to estimate the first term. Using the first equation in (4.1), we get

$$
\begin{aligned}
& \varepsilon \nu^{2}\left\langle\left\langle\operatorname{div} \widetilde{v},\left(\partial_{t}+v \cdot \nabla\right) \widetilde{p}\right\rangle\right\rangle=-\nu^{2}\left\langle\left\langle\operatorname{div} \widetilde{v}, g_{1}^{-1} \operatorname{div} \widetilde{v}\right\rangle\right\rangle \\
& \quad+\nu^{2} \kappa\left\langle\left\langle\operatorname{div} \widetilde{v}, g_{1}^{-1} \operatorname{div}\left(\beta_{1} \nabla \widetilde{\theta}\right)\right\rangle\right\rangle+\varepsilon \nu^{2}\left\langle\left\langle\operatorname{div} \widetilde{v}, g_{1}^{-1} f_{1}\right\rangle\right\rangle .
\end{aligned}
$$

Then, the analysis establishing (4.34) also gives

$$
\begin{aligned}
& \varepsilon \nu^{2} \mid\left\langle\left\langle\operatorname{div} \widetilde{v},\left(\partial_{t}+v \cdot \nabla\right) \widetilde{p}\right\rangle\right| \\
& \quad \leqslant C_{0} e^{T C}\left\{\nu^{2}\left\|\left(\operatorname{div} \widetilde{v}, \kappa \nabla^{2} \widetilde{\theta}\right)\right\|_{L^{2}\left(\Omega_{t}\right)}^{2}+\nu^{2} \kappa\|(\nabla \widetilde{\theta}, \varepsilon \nabla \widetilde{v})\|_{C_{t}^{0} L^{2}}^{2}+\left\|f_{1}\right\|_{L_{t}^{1} L^{2}}^{2}\right\},
\end{aligned}
$$

which in turn is bounded by the right-hand side of (4.32). The proof is complete.

We thus have proved a closed system of estimates. Indeed, by taking appropriate combinations of the previous estimates, we see that there exists a constant $C_{0}$ depending only on $R_{0}$ and a constant $C$ depending only on $R$ such that for all $t \in[0, T]$ and all $\lambda \geqslant 1$, the norm $N(t)$ (as defined in (4.28) ) satisfies the estimate

$$
N(t)^{2} \leqslant C_{0} e^{T C} N(0)^{2}+\lambda C \int_{0}^{t} N(\tau)^{2} d \tau+\frac{C}{\lambda} N(t)^{2}+C F(T)^{2},
$$

where $F(T):=\int_{0}^{t}\left\|\left(f_{1}, f_{2}\right)\right\|_{H_{\varepsilon \nu}^{1}}+\left\|f_{3}\right\|_{H_{\nu}^{1}} d t$.

Again, take $\lambda$ large enough and apply the Gronwall's lemma. The proof of Theorem 4.3 is complete since $\|\widetilde{U}\|_{\mathcal{H}_{a}^{0}(T)} \leqslant N(T)$. 


\section{High FRequency REGime}

To establish Theorem 2.7 the crucial part consists in obtaining a priori estimates in Sobolev norms independent of $a \in A$. Theorem 4.3 provides the basic $L^{2}$ estimates. However, the estimates of the derivatives also require a careful analysis. Indeed, the classical approach, which consists in differentiating the equations, certainly fails since it reveals unbounded terms in $\varepsilon^{-1}$. However, one can follow this strategy in the high frequency regime where the parabolic behavior prevails.

More precisely, given a smooth solution $U=(p, v, \theta)$ to (2.1), we will estimate the $\mathcal{H}_{a}^{\mathrm{s}}(T)$ norm of $\left(I-J_{h}\right) U$, where $\mathcal{H}_{a}^{\mathrm{s}}(T)$ is as defined in Definition 2.4 and, in keeping with the notations of $\sqrt{3}$. $\left\{J_{h} \mid h \in[0,1]\right\}$ is a Friedrichs' mollifier and $\Lambda^{s}:=(I-\Delta)^{s / 2}$. In order to make our energy estimates applicable, the main difficulty is to verify that the commutator of $\left(I-J_{h}\right) \Lambda^{s}$ and the equations (2.1) can be seen as a source term. To do so we first note that for $h=\mathcal{O}(\varepsilon)$, one can gain an extra factor $\varepsilon$ in the commutator estimates [see (5.2) below]. Yet, this costs a derivative. To compensate this loss of derivative, we use in an essential way the parabolic behavior of the equations. Consequently, we search $h$ under the form $c(\mu, \kappa) \varepsilon$. Since for our purposes the main smoothing effect concerns the penalized terms $\operatorname{div} v$ and $\nabla p$, we take $c(\mu, \kappa)=\sqrt{\mu+\kappa}$.

Proposition 5.1. Given an integer $s>1+d / 2$, there exists a continuous non-decreasing function $C$ such that for all $a=(\varepsilon, \mu, \kappa) \in A$, all $T \in[0,1]$ and all $U=(p, v, \theta) \in C^{1}\left([0, T] ; H^{\infty}(\mathbb{D})\right)$ satisfying (2.1),

$$
\left\|\left(I-J_{\varepsilon \nu}\right) U\right\|_{\mathcal{H}_{a}^{\mathrm{s}}(T)} \leqslant C\left(\Omega_{0}\right) e^{\sqrt{T} C(\Omega)},
$$

where $\nu:=\sqrt{\mu+\kappa}, \Omega_{0}:=\|U(0)\|_{\mathcal{H}_{a, 0}^{s}}, \Omega:=\|U\|_{\mathcal{H}_{a}^{s}(T)}$ (see Definition 2.4).

Remark 5.2. Note that we establish estimates for the exact solutions of (2.1) and we do not estimate the solutions of the linearized system (4.1).

5.1. Preliminaries. To avoid interruptions of the proofs later on, we now collect a few nonlinear estimates we use throughout this section.

Recall that $\|\cdot\|_{H^{\sigma}}:=\|\cdot\|_{H^{\sigma-1}}+\varrho\|\cdot\|_{H^{\sigma}}$.

Lemma 5.3. Let $s>1+d / 2$. There exists a constant $K$ such that for all $h \in[0,1]$, all $\varrho \geqslant 0$, all $f \in H^{s+1}(\mathbb{D})$ and all $u \in H^{S}(\mathbb{D})$, one has

$$
\left\|\left[f,\left(I-J_{h}\right) \Lambda^{s}\right] u\right\|_{H_{\varrho}^{1}} \leqslant(\varrho+h) K\left\{\|f\|_{W^{1, \infty}}\|u\|_{H^{s}}+\|f\|_{H^{s+1}}\|u\|_{L^{\infty}}\right\},
$$

where $\|f\|_{W^{1, \infty}}:=\|f\|_{L^{\infty}}+\|\nabla f\|_{L^{\infty}}$.

Remark 5.4. This inequality provides a way to gain an extra factor $h$ [with $\varrho=h$ ] or a derivative [with $\varrho=1$ ]. In this respect it is like the estimate (3.11), to which we will return in a moment (see Lemma 6.6).

Proof. To prove this result we need a tame estimate version of (3.10). We use the following result, which is Proposition 3.6.A in [40] (see also [25]). 
Let $m>0$. For all Fourier multiplier $\mathcal{Q}$ with symbol $q \in S^{m}$ and for all $\sigma \geqslant 0$, we have

$$
\|[f, \mathcal{Q}] u\|_{H^{\sigma}} \leqslant K\|f\|_{W^{1, \infty}}\|u\|_{H^{\sigma+m-1}}+K\|f\|_{H^{m+\sigma}}\|u\|_{L^{\infty}},
$$

where the constant $K$ depends only on $m, s, \sigma, d$ and a finite number of semi-norms of $q$ in $S^{m}$.

Since the support of the symbol $1-\jmath_{h}$ is limited by $|\xi| \geqslant 1 / h$, the most direct estimates show that $\left\{h^{-1}(1-\jmath(h \xi))\langle\xi\rangle^{s} \mid h \in[0,1]\right\}$ is uniformly bounded in the symbol class $S^{s+1}$. Thus, the commutator estimate (5.3), applied with $(m, \sigma):=(s+1,0)$, implies

$$
\left\|\left[f,\left(I-J_{h}\right) \Lambda^{s}\right] u\right\|_{L^{2}} \lesssim h\left(\|f\|_{W^{1, \infty}}\|u\|_{H^{s}}+\|f\|_{H^{s+1}}\|u\|_{L^{\infty}}\right) .
$$

On the other hand, the family $\left\{(1-\jmath(h \xi))\langle\xi\rangle^{s} \mid h \in[0,1]\right\}$ is uniformly bounded in $S^{s}$, so that (5.3) applied with $(m, \sigma)=(s, 1)$ implies

$$
\left\|\left[f,\left(I-J_{h}\right) \Lambda^{s}\right] u\right\|_{H^{1}} \lesssim\|f\|_{W^{1, \infty}}\|u\|_{H^{s}}+\|f\|_{H^{s+1}}\|u\|_{L^{\infty}} .
$$

Combining (5.4) with (5.5) multiplied by $\varrho$, yields (5.2).

We next prove two Moser-type estimates for the norms $\|\cdot\|_{H_{\varrho}^{\sigma}}$.

Lemma 5.5. Let $s>1+d / 2$. There exists a constant $K$ such that for all $\varrho \geqslant 0$ and for all $u_{i} \in H_{\varrho}^{S}(\mathbb{D})$,

$$
\left\|u_{1} u_{2}\right\|_{H_{\varrho}^{s}} \leqslant K\left\|u_{1}\right\|_{H_{\varrho}^{s}}\left\|u_{2}\right\|_{H_{\varrho}^{s}} .
$$

This result extends to vector valued functions.

Proof. Using the standard tame estimate for products at orders $s-1$ and $s$, we get

$$
\begin{aligned}
\left\|u_{1} u_{2}\right\|_{H^{s-1}} & \lesssim\left\|u_{1}\right\|_{L^{\infty}}\left\|u_{2}\right\|_{H^{s-1}}+\left\|u_{1}\right\|_{H^{s-1}}\left\|u_{2}\right\|_{L^{\infty}}, \\
\left\|u_{1} u_{2}\right\|_{H^{s}} & \lesssim\left\|u_{1}\right\|_{L^{\infty}}\left\|u_{2}\right\|_{H^{s}}+\left\|u_{1}\right\|_{H^{s}}\left\|u_{2}\right\|_{L^{\infty}} .
\end{aligned}
$$

Using the definition $\|\cdot\|_{H^{s}}:=\|\cdot\|_{H^{s-1}}+\varrho\|\cdot\|_{H^{s}}$, and putting the parameter $\varrho$ in appropriate spots yields

$$
\left\|u_{1} u_{2}\right\|_{H_{\varrho}^{s}} \lesssim\left\|u_{1}\right\|_{L^{\infty}}\left\|u_{2}\right\|_{H_{\varrho}^{s}}+\left\|u_{1}\right\|_{H_{\varrho}^{s}}\left\|u_{2}\right\|_{L^{\infty}} .
$$

Since $s-1>d / 2$, the Sobolev Theorem implies $\|u\|_{L^{\infty}} \lesssim\|u\|_{H^{s-1}} \lesssim\|u\|_{H_{e}^{s}}$. Which completes the proof.

Lemma 5.6. Let $s>1+d / 2$ and $F: \mathbb{R}^{n} \rightarrow \mathbb{C}$ be a $C^{\infty}$ function such that $F(0)=0$. Then, for all $\varrho \geqslant 0$ and all $u \in H_{\varrho}^{S}(\mathbb{D})$ with values in $\mathbb{R}^{n}$,

$$
\|F(u)\|_{H_{\varrho}^{s}} \leqslant C\left(\|u\|_{H_{\varrho}^{s}}\right),
$$

where $C(\cdot)$ depends only on a finite number of semi-norms of $F$ in $C^{\infty}$. 
Proof. Recall that, for all $\sigma_{0}>d / 2$ and all $C^{\infty}$ function $F$ satisfying $F(0)=0$,

$$
\|F(u)\|_{H^{\sigma_{0}}} \leqslant C\left(\|u\|_{L^{\infty}}\right)\|u\|_{H^{\sigma_{0}}}
$$

Using this estimate at orders $s-1$ and $s$, one has

$$
\|F(u)\|_{H_{\varrho}^{s}} \leqslant C\left(\|u\|_{L^{\infty}}\right)\|u\|_{H_{\varrho}^{s}} .
$$

This in turn implies the desired estimate.

Lemma 5.7. Let $s>1+d / 2, F: \mathbb{R}^{n} \rightarrow \mathbb{C}$ be a $C^{\infty}$ function such that $F(0)=0$ and $\mathcal{Q}$ be a Fourier multiplier with symbol $q \in S^{s}$. For all vector-valued function $u \in H^{\mathrm{S}}(\mathbb{D})$ one has

$$
\left\|\mathcal{Q}(F(u))-F^{\prime}(u) \mathcal{Q} u\right\|_{H^{1}} \leqslant C\left(\|u\|_{H^{s}}\right),
$$

where $F^{\prime}$ is the differential of $F$ and $C(\cdot)$ is a smooth non-decreasing function depending only on $s, d$, a finite number of semi-norms of $q$ in $S^{s}$ and a finite number of semi-norms of $F$ in $C^{\infty}$.

Proof. To establish (5.10) we use the para-differential calculus of Bony [2]. Denote by $T_{f}$ the operator of para-multiplication by $f$. Starting from

$$
F(u)=T_{F^{\prime}(u)} u+R\left(u ; x, D_{x}\right) u,
$$

where $R\left(u ; x, D_{x}\right)$ is a smoothing operator (see (5.11)), we obtain $\mathcal{Q}(F(u))-F^{\prime}(u) \mathcal{Q} u=\left(T_{F^{\prime}(u)}-F^{\prime}(u)\right) \mathcal{Q} u+\left[\mathcal{Q}, T_{F^{\prime}(u)}\right] u+\mathcal{Q} R\left(u ; x, D_{x}\right) u$. The claim then follows from the bounds (3.16)-(3.18) and the estimate

$$
\left\|R\left(u ; x, D_{x}\right) u\right\|_{H^{s+1}} \leqslant C\left(\|u\|_{H^{s}}\right) \text {. }
$$

See [17, Th. 10.3.1] and [17, Corollary 9.3.6] for the proof of (5.11).

5.2. Localization in the high frequency region. To simplify the presentation, we fix a real number $s$ strictly greater than $1+d / 2$.

To proceed further, we need some more terminology.

Notation 5.8. For all $h \in[0,2]$, define

$$
\mathcal{Q}_{h}:=\left(I-J_{h}\right) \Lambda^{s} .
$$

Hereafter, the parameter $h$ is a product $\varepsilon \nu$ with $(\varepsilon, \nu) \in[0,1] \times[0,2]$.

Introduce next the commutator of the equations (2.1) and $\mathcal{Q}_{\varepsilon \nu}$.

Notation 5.9. Given $a=(\varepsilon, \mu, \kappa) \in A, \nu \in[0,2]$ and $U=(p, v, \theta)$, set

$$
\begin{aligned}
f_{1, \mathrm{HF}}^{a, \nu}(U) & :=\left[g_{1}(\phi), \mathcal{Q}_{\varepsilon \nu}\right] \partial_{t} p+\left[g_{1}(\phi) v, \mathcal{Q}_{\varepsilon \nu}\right] \cdot \nabla p-\frac{\kappa}{\varepsilon}\left[B_{1}(\phi), \mathcal{Q}_{\varepsilon \nu}\right] \theta, \\
f_{2, \mathrm{HF}}^{a, \nu}(U) & :=\left[g_{2}(\phi), \mathcal{Q}_{\varepsilon \nu}\right] \partial_{t} v+\left[g_{2}(\phi) v, \mathcal{Q}_{\varepsilon \nu}\right] \cdot \nabla v-\mu\left[B_{2}(\phi), \mathcal{Q}_{\varepsilon \nu}\right] v, \\
f_{3, \mathrm{HF}}^{a, \nu}(U) & :=\left[g_{3}(\phi), \mathcal{Q}_{\varepsilon \nu}\right] \partial_{t} \theta+\left\{g_{3}(\phi) v \cdot \nabla \theta ; \mathcal{Q}_{\varepsilon \nu}\right\}-\kappa\left[B_{3}(\phi), \mathcal{Q}_{\varepsilon \nu}\right] \theta,
\end{aligned}
$$

where $\phi$ is a shorthand notation for $(\theta, \varepsilon p)$ and

$\left\{g_{3}(\phi) v \cdot \nabla \theta ; \mathcal{Q}_{\varepsilon \nu}\right\}:=g_{3}(\phi) v \cdot \nabla \mathcal{Q}_{\varepsilon \nu} \theta+g_{3}(\phi) \nabla \theta \cdot \mathcal{Q}_{\varepsilon \nu} v-\mathcal{Q}_{\varepsilon \nu}\left(g_{3}(\phi) v \cdot \nabla \theta\right)$. 
Remark 5.10. For the purpose of proving estimates independent of $\kappa$, we do not consider the exact commutator of the third equation in (2.1) with $\mathcal{Q}_{\varepsilon \nu}$.

Let us recall that

$$
\begin{aligned}
& B_{1}(\phi):=\chi_{1}(\varepsilon p) \operatorname{div}(\beta(\theta) \nabla \cdot), \\
& B_{2}(\phi):=\chi_{2}(\varepsilon p) \operatorname{div}(2 \zeta(\theta) D \cdot)+\chi_{2}(\varepsilon p) \nabla(\eta(\theta) \operatorname{div} \cdot), \\
& B_{3}(\phi):=\chi_{3}(\varepsilon p) \operatorname{div}(\beta(\theta) \nabla \cdot) .
\end{aligned}
$$

The following lemma shows that $f_{\mathrm{HF}}^{a, \nu}$ can be seen as a source term.

Lemma 5.11. There exists a smooth non-decreasing function $C=C(\cdot)$ such that for all $a \in A$, all $T \geqslant 0$, all $\nu \in[0,2]$ and all vector valued function $U:=(p, v, \theta) \in C^{1}\left([0, T] ; H^{\infty}(\mathbb{D})\right)$,

$$
\begin{aligned}
& \left\|f_{1, \mathrm{HF}}^{a, \nu}(U)\right\|_{H_{\varepsilon \nu}^{1}} \leqslant C(R)\left\{1+\left\|\varepsilon \partial_{t} p\right\|_{H_{\nu}^{s}}+\kappa\|\theta\|_{H^{s+2}}\right\}, \\
& \left\|f_{2, \mathrm{HF}}^{a, \nu}(U)\right\|_{H_{\varepsilon \nu}^{1}} \leqslant C(R)\left\{1+\left\|\varepsilon \partial_{t} v\right\|_{H_{\nu}^{s}}+\mu\|\varepsilon v\|_{H^{s+2}}\right\}, \\
& \left\|f_{3, \mathrm{HF}}^{a, \nu}(U)\right\|_{H_{\nu}^{1}} \leqslant C(R)\left\{1+\left\|\partial_{t} \theta\right\|_{H_{\nu}^{s}}+\kappa\|\theta\|_{H^{s+2}}\right\},
\end{aligned}
$$

where $R:=\|(p, v)\|_{H_{\varepsilon \nu}^{s+1}}+\|\theta\|_{H_{\nu}^{s+1}}$.

Remark 5.12. We will apply this lemma with $\nu:=\sqrt{\mu+\kappa}$. Yet, we prove estimates independent of $\nu \in[0,2]$ to explain why the frequency space is cut around $|\xi| \approx 1 /(\varepsilon \sqrt{\mu+\kappa})$.

Proof. We make intensive use of the following obvious observations. Firstly, using the Sobolev embedding Theorem and the very definition of the norms $\|\cdot\|_{H_{\varrho}^{\sigma}}$, one has

$$
\|u\|_{L^{\infty}} \lesssim\|u\|_{H^{s-1}} \leqslant\|u\|_{H_{\nu}^{s}} \quad \text { and } \quad\|u\|_{W^{1, \infty}} \lesssim\|u\|_{H^{s}} \leqslant\|u\|_{H_{\nu}^{s+1}} .
$$

Since $\varepsilon \leqslant 1$ and $\nu \leqslant 2$, directly from the definition of $\|\cdot\|_{H_{\nu}^{\sigma}}$, one has

$$
\nu\|u\|_{H^{\sigma}} \leqslant\|u\|_{H_{\nu}^{\sigma}} \leqslant 3\|u\|_{H^{\sigma}} \quad \text { and } \quad\|\varepsilon u\|_{H_{\nu}^{s+1}} \leqslant\|u\|_{H_{\varepsilon \nu}^{s+1}} .
$$

Note the following corollary of the second inequality: $\|(\varepsilon p, \varepsilon v)\|_{H_{\nu}^{s+1}} \leqslant R$. This in turn implies $\|\phi\|_{H_{\nu}^{s+1}}:=\|(\theta, \varepsilon p)\|_{H_{\nu}^{s+1}} \leqslant R$.

STEP 1: Estimate for $f_{1, \mathrm{HF}}^{a, \nu}(\mathrm{U})$. a) We begin by proving that

$$
\left\|\left[g_{1}(\phi), \mathcal{Q}_{\varepsilon \nu}\right] \partial_{t} p\right\|_{H_{\varepsilon \nu}^{1}} \leqslant C(R)\left\|\varepsilon \partial_{t} p\right\|_{H_{\nu}^{s}} .
$$

Applying the commutator estimate (5.2) with $h=\varrho=\varepsilon \nu$, we have

$$
\begin{aligned}
\left\|\left[g_{1}(\phi), \mathcal{Q}_{\varepsilon \nu}\right] \partial_{t} p\right\|_{H_{\varepsilon \nu}^{1}} & \lesssim \varepsilon \nu\left\|\tilde{g}_{1}(\phi)\right\|_{W^{1, \infty}}\left\|\partial_{t} p\right\|_{H^{s}}+\varepsilon \nu\left\|\tilde{g}_{1}(\phi)\right\|_{H^{s+1}}\left\|\partial_{t} p\right\|_{L^{\infty}} \\
& \lesssim\left\|\tilde{g}_{1}(\phi)\right\|_{W^{1, \infty}}\left\|\varepsilon \partial_{t} p\right\|_{H_{\nu}^{s}}+\left\|\tilde{g}_{1}(\phi)\right\|_{H_{\nu}^{s+1}}\left\|\varepsilon \partial_{t} p\right\|_{L^{\infty}},
\end{aligned}
$$

where $\tilde{g}_{1}$ is defined by $\tilde{g}_{1}=g_{1}-g_{1}(0)$. The estimates (5.12) imply that the right side is less than $K\left\|\tilde{g}_{1}(\phi)\right\|_{H_{\nu}^{s+1}}\left\|\varepsilon \partial_{t} p\right\|_{H_{\nu}^{s}}$. Using Lemma 5.6 we obtain $\left\|\tilde{g}_{1}(\phi)\right\|_{H_{\nu}^{s+1}} \leqslant C\left(\|\phi\|_{H_{\nu}^{s+1}}\right) \leqslant C(R)$. This proves (5.14). 
b) Next, we prove that

$$
\left\|\left[g_{1}(\phi) v, \mathcal{Q}_{\varepsilon \nu}\right] \cdot \nabla p\right\|_{H_{\varepsilon \nu}^{1}} \leqslant C(R)
$$

Again, this follows from the commutator estimate (5.2) applied with $h=$ $\varrho=\varepsilon \nu$. Indeed, it yields

$\left\|\left[g_{1}(\phi) v, \mathcal{Q}_{\varepsilon \nu}\right] \cdot \nabla p\right\|_{H_{\varepsilon \nu}^{1}} \lesssim\left\|\tilde{g}_{1}(\phi) v\right\|_{W^{1, \infty}}\|\varepsilon \nabla p\|_{H_{\nu}^{s}}+\left\|\varepsilon \tilde{g}_{1}(\phi) v\right\|_{H_{\nu}^{s+1}}\|\nabla p\|_{L^{\infty}}$.

The first term in the right-hand side is estimated by $C(R)$ since

$$
\left\|\tilde{g}_{1}(\phi) v\right\|_{W^{1, \infty}} \leqslant C\left(\|\phi\|_{W^{1, \infty}},\|v\|_{W^{1, \infty}}\right) \leqslant C\left(\|\phi\|_{H^{s}},\|v\|_{H^{s}}\right) \leqslant C(R),
$$

and $\|\varepsilon \nabla p\|_{H_{\nu}^{s}} \leqslant\|p\|_{H_{\varepsilon \nu}^{s+1}} \leqslant R$.

It thus remains to estimate $\left\|\varepsilon \tilde{g}_{1}(\phi) v\right\|_{H_{\nu}^{s+1}}$. To do so use Lemma [5.5] and 5.6, to obtain

$$
\left\|\varepsilon \tilde{g}_{1}(\phi) v\right\|_{H_{\nu}^{s+1}} \lesssim\left\|\tilde{g}_{1}(\phi)\right\|_{H_{\nu}^{s+1}}\|\varepsilon v\|_{H_{\nu}^{s+1}} \leqslant C(R) R .
$$

c) Let us prove that

$$
\frac{\kappa}{\varepsilon}\left\|\left[B_{1}(\phi), \mathcal{Q}_{\varepsilon \nu}\right] \theta\right\|_{H_{\varepsilon \nu}^{1}} \leqslant C(R)\|\kappa \theta\|_{H^{s+2}} .
$$

By definition $B_{1}(\phi)=\chi_{1}(\varepsilon p) \operatorname{div}(\beta(\theta) \nabla \cdot)$, so that one can decompose the commutator $\left[B_{1}(\phi), \mathcal{Q}_{\varepsilon \nu}\right] \theta$ as

$$
\left[\mathcal{E}_{1}(\phi), \mathcal{Q}_{\varepsilon \nu}\right] \Delta \theta+\left[\mathcal{E}_{2}(\phi, \nabla \phi), \mathcal{Q}_{\varepsilon \nu}\right] \cdot \nabla \theta,
$$

where $\mathcal{E}_{1}(\phi)=\chi_{1}(\varepsilon p) \beta(\theta)$ and $\mathcal{E}_{2}(\phi, \nabla \phi)=\chi_{1}(\varepsilon p) \nabla \beta(\theta)=\chi_{1}(\varepsilon p) \beta^{\prime}(\theta) \nabla \theta$. The commutator estimate (5.2) [applied with $h=\varrho=\varepsilon \nu$ ] implies

$$
\begin{aligned}
\left\|\left[\mathcal{E}_{1}, \mathcal{Q}_{\varepsilon \nu}\right] \Delta \theta\right\|_{H_{\varepsilon \nu}^{1}} & \lesssim \varepsilon\left\|\tilde{\mathcal{E}}_{1}\right\|_{W^{1, \infty}}\|\Delta \theta\|_{H_{\nu}^{s}}+\varepsilon\left\|\tilde{\mathcal{E}}_{1}\right\|_{H_{\nu}^{s+1}}\|\Delta \theta\|_{L^{\infty}}, \\
\left\|\left[\mathcal{E}_{2}, \mathcal{Q}_{\varepsilon \nu}\right] \cdot \nabla \theta\right\|_{H_{\varepsilon \nu}^{1}} & \lesssim \varepsilon\left\|\nu \mathcal{E}_{2}\right\|_{W^{1, \infty}}\|\nabla \theta\|_{H^{s}}+\varepsilon\left\|\mathcal{E}_{2}\right\|_{H_{\nu}^{s+1}}\|\nabla \theta\|_{L^{\infty}},
\end{aligned}
$$

where $\tilde{\mathcal{E}}_{1}:=\mathcal{E}_{1}-\mathcal{E}_{1}(0)$. We gather

$$
\begin{array}{ll}
\left\|\tilde{\mathcal{E}}_{1}(\phi)\right\|_{W^{1, \infty}} \leqslant C\left(\|\phi\|_{H^{s}}\right) \leqslant C(R), & \|\Delta \theta\|_{H_{\nu}^{s}} \lesssim\|\theta\|_{H^{s+2}}, \\
\left\|\tilde{\mathcal{E}}_{1}(\phi)\right\|_{H_{\nu}^{s+1}} \leqslant C\left(\|\phi\|_{H_{\nu}^{s+1}}\right) \leqslant C(R), & \|\Delta \theta\|_{L^{\infty}} \lesssim\|\theta\|_{H^{s+2}}, \\
\left\|\nu \mathcal{E}_{2}(\phi, \nabla \phi)\right\|_{W^{1, \infty}} \leqslant C\left(\|\phi\|_{H_{\nu}^{s+1}}\right) \leqslant C(R), & \|\nabla \theta\|_{H^{s}} \leqslant\|\theta\|_{H^{s+2}}, \\
\left\|\mathcal{E}_{2}(\phi, \nabla \phi)\right\|_{H_{\nu}^{s+1}} \leqslant C(R)\|\theta\|_{H^{s+2}}, & \|\nabla \theta\|_{L^{\infty}} \lesssim\|\theta\|_{H^{s}} \leqslant R .
\end{array}
$$

The first six inequalities follows from Lemma 5.6, the Sobolev Theorem and the estimates (5.12)-(5.13). To estimate $\left\|\tilde{\mathcal{E}}_{2}(\phi, \nabla \phi)\right\|_{H_{\nu}^{s+1}}$, we first use Lemma 5.5, to obtain

$$
\left\|\mathcal{E}_{2}(\phi, \nabla \phi)\right\|_{H_{\nu}^{s+1}} \lesssim\left(1+\left\|\tilde{\chi_{1}}(\varepsilon p)\right\|_{H_{\nu}^{s+1}}\right)\left(1+\left\|\tilde{\beta^{\prime}}(\theta)\right\|_{H_{\nu}^{s+1}}\right)\|\nabla \theta\|_{H_{\nu}^{s+1}}
$$

and next use Lemma 5.6 to bound the $H_{\nu}^{s+1}$-norms of $\tilde{\chi}_{1}(\varepsilon \theta)$ and $\tilde{\beta}^{\prime}(\theta)$ by $C(R)$. This yields the desired bound since $\|\nabla \theta\|_{H_{\nu}^{s+1}} \lesssim\|\theta\|_{H^{s+2}}$.

The claim (5.16) then easily follows from these estimates. 
STEP 2: Estimate for $f_{2, \mathrm{HF}}^{a, \nu}(U)$. Note that one can obtain $f_{2, \mathrm{HF}}^{a, \nu}(U)$ from $f_{1, \mathrm{HF}}^{a, \nu}(U)$ by replacing $p$ by $v$ and $\theta$ by $\varepsilon v$. Therefore, we are back in the situation of the previous step, and hence conclude that

$$
\begin{aligned}
\left\|\left[g_{2}(\phi), \mathcal{Q}_{\varepsilon \nu}\right] \partial_{t} v\right\|_{H_{\varepsilon \nu}^{1}} & \leqslant C(R)\left\|\varepsilon \partial_{t} v\right\|_{H_{\nu}^{s}}, \\
\left\|\left[g_{2}(\phi) v, \mathcal{Q}_{\varepsilon \nu}\right] \cdot \nabla v\right\|_{H_{\varepsilon \nu}^{1}} & \leqslant C(R), \\
\mu\left\|\left[B_{2}(\phi), \mathcal{Q}_{\varepsilon \nu}\right] v\right\|_{H_{\varepsilon \nu}^{1}} & \leqslant C(R)\|\mu \varepsilon v\|_{H^{s+2}} .
\end{aligned}
$$

STEP 3: Estimate for $f_{3, \mathrm{HF}}^{a, \nu}(U)$. The estimates for the first and the last terms in $f_{3, \mathrm{HF}}^{a, \nu}(U)$ can be deduced by following the previous analysis. We only indicate the point at which the argument differs: we use the commutator estimate (5.5) with $h=\varepsilon \nu$ and $\varrho=\nu$ (instead of $\varrho=\varepsilon \nu)$.

Let us concentrate on the second term. We claim that

$$
\left\|\left\{g_{3}(\phi) v \cdot \nabla \theta ; \mathcal{Q}_{\varepsilon \nu}\right\}\right\|_{H_{\nu}^{1}} \leqslant C(R) .
$$

We first decompose $\left\{g_{3}(\phi) v \cdot \nabla \theta ; \mathcal{Q}_{\varepsilon \nu}\right\}$ as

$$
\left[g_{3}(\phi), \mathcal{Q}_{\varepsilon \nu}\right] v \cdot \nabla \theta+g_{3}(\phi) Z
$$

where $Z:=v \cdot \nabla \mathcal{Q}_{\varepsilon \nu} \theta+\nabla \theta \cdot \mathcal{Q}_{\varepsilon \nu} v-\mathcal{Q}_{\varepsilon \nu}(v \cdot \nabla \theta)$

Applying the commutator estimate (5.2) with $h=\varepsilon \nu$ and $\varrho=\nu$, and using the estimates (5.12), we get

$$
\left\|\left[g_{3}(\phi), \mathcal{Q}_{\varepsilon \nu}\right] v \cdot \nabla \theta\right\|_{H_{\nu}^{1}} \lesssim\left\|\tilde{g}_{3}(\phi)\right\|_{H_{\nu}^{s+1}}\|v \cdot \nabla \theta\|_{H_{\nu}^{s}} \cdot
$$

Using Lemma 5.5] and [5.6. we find that the right-hand side of the previous estimate is dominated by $C\left(\|\phi\|_{H_{\nu}^{s+1}}\right)\|v\|_{H_{\nu}^{s}}\|\nabla \theta\|_{H_{\nu}^{s}} \leqslant C(R)$.

Since $\left\|g_{3}(\phi) Z\right\|_{H_{\nu}^{1}} \leqslant\left\|g_{3}(\phi)\right\|_{W^{1, \infty}}\|Z\|_{H_{\nu}^{1}} \leqslant C(R)\|Z\|_{H_{\nu}^{1}}$, to control the $H_{\nu}^{1}$-norm of the second term in (5.19), only the estimate of $\|Z\|_{H_{\nu}^{1}}$ is missing. We split $\|Z\|_{H_{\nu}^{1}}$ as $\|Z\|_{L^{2}}+\nu\|Z\|_{H^{1}}$, and we prove that $\|Z\|_{L^{2}}$ and $\nu\|Z\|_{H^{1}}$ are both estimated by $C(R)$. To estimate $\|Z\|_{L^{2}}$, note that

$$
Z=\nabla \theta \cdot \mathcal{Q}_{\varepsilon \nu} v+\left[v, \mathcal{Q}_{\varepsilon \nu}\right] \cdot \nabla \theta .
$$

The second term in the right-hand side is estimated by way of the commutator estimate (3.10). Indeed, applying Lemma 3.4 with $\sigma_{0}=m=s$ and $\sigma=s-1$, we get

$$
\left\|\left[v, \mathcal{Q}_{\varepsilon \nu}\right] \cdot \nabla \theta\right\|_{L^{2}} \lesssim\|v\|_{H^{s}}\|\nabla \theta\|_{H^{s-1}} \leqslant\|v\|_{H^{s}}\|\theta\|_{H^{s}} \leqslant R^{2} .
$$

As regards the first term in the right-hand side of (5.20), write

$$
\left\|\nabla \theta \cdot \mathcal{Q}_{\varepsilon \nu} v\right\|_{L^{2}} \leqslant\|\nabla \theta\|_{L^{\infty}}\left\|\mathcal{Q}_{\varepsilon \nu} v\right\|_{L^{2}} \lesssim\|\theta\|_{H^{s}}\|v\|_{H^{s}} \leqslant R^{2} .
$$

Moving to the estimate of $\nu\|Z\|_{H^{1}}$, remark that

$$
\nu Z=F^{\prime}(u) \mathcal{Q}_{\varepsilon \nu} u-\mathcal{Q}_{\varepsilon \nu}(F(u)),
$$

with $u=\left(u_{1}, u_{2}\right):=(v, \nu \nabla \theta)$ and $F(u)=u_{1} u_{2}$. Hence, (15.10) yields

$$
\nu\|Z\|_{H^{1}} \leqslant C\left(\|(v, \nu \nabla \theta)\|_{H^{s}}\right) \leqslant C\left(\|v\|_{H^{s}}+\|\theta\|_{H_{\nu}^{s+1}}\right) \leqslant C(R) .
$$


The previous estimates imply that $\|Z\|_{H_{\nu}^{1}}$ is controlled by $C(R)$. This completes the proof of Lemma 5.11

Remark 5.13. Let us explain the reason why we assume that $\beta$ depends only on $\theta$. Had we worked instead with general coefficient $\beta$ depending also on $\varepsilon p$, the corresponding inequality (5.18) would have involved $\|\varepsilon \nabla p\|_{H_{\nu}^{s+1}}$. The problem presents itself: $\|\varepsilon p\|_{L^{1}\left(0, T ; H_{\nu}^{s+2}\right)}$ is not controlled by the norm $\|(p, v, \theta)\|_{\mathcal{H}_{a}^{s}(T)}$. It is possible to get around the previous problem, yet we do not address this question.

In view of Lemma 5.11 we are led to estimate $\partial_{t} \psi$ where $\psi:=(\theta, \varepsilon p, \varepsilon v)$.

Lemma 5.14. There exists a continuous non-decreasing function $C(\cdot)$ such that for all $a \in A$, all $T \geqslant 0$, all $\nu \in[0,2]$ and all $(p, v, \theta) \in \mathcal{H}_{a}^{\mathrm{s}}(T)$ solving (2.1), the function $\psi:=(\theta, \varepsilon p, \varepsilon v)$ satisfies

$$
\left\|\partial_{t} \psi\right\|_{H_{\nu}^{s}} \leqslant C(R)\left\{1+R^{\prime}\right\},
$$

where

$$
\begin{aligned}
R & :=\|(p, v)\|_{H_{\varepsilon \nu}^{s+1}}+\|\theta\|_{H_{\nu}^{s+1}}, \\
R^{\prime} & :=\nu\|(\operatorname{div} v, \nabla p)\|_{H^{s}}+\sqrt{\mu}\|\nabla v\|_{H_{\varepsilon \nu}^{s+1}}+\sqrt{\kappa}\|\nabla \theta\|_{H_{\nu}^{s+1}} .
\end{aligned}
$$

Remark 5.15. This estimate plays a key role for the purpose of proving estimates independent of $\mu$ and $\kappa$. Indeed, this is the only step in which we use the additional smoothing effect for $\operatorname{div} v$ and $\nabla p$. More precisely, the fact that the estimate (5.21) is tame [linear in $R^{\prime}$ ] allows us to control $\left\|\partial_{t} \psi\right\|_{L^{2}\left(0, T ; H_{\nu}^{s}\right)}$ by $C\left(\|(p, v, \theta)\|_{\mathcal{H}_{a}^{s}(T)}\right)$ for all $\nu \leqslant \sqrt{\mu+\kappa}$.

Proof. In order to establish (5.21), observe that

$$
G(\phi)\left(\partial_{t} \psi+v \cdot \nabla \psi\right)+L U+B_{\mu, \kappa}(\phi) \psi=0,
$$

where $\psi$ and $U$ are identified with $(\varepsilon p, \varepsilon v, \theta)^{t}$ and $(p, v, \theta)^{t}$, respectively, and

$$
G:=\left(\begin{array}{ccc}
g_{1} & 0 & 0 \\
0 & g_{2} I_{d} & 0 \\
0 & 0 & g_{3}
\end{array}\right), L:=\left(\begin{array}{ccc}
0 & \operatorname{div} & 0 \\
\nabla & 0 & 0 \\
0 & \operatorname{div} & 0
\end{array}\right), B_{\mu, \kappa}:=\left(\begin{array}{ccc}
0 & 0 & \kappa B_{1} \\
0 & \mu B_{2} & 0 \\
0 & 0 & \kappa B_{3}
\end{array}\right) .
$$

Since $\max \{\mu, \kappa\} \leqslant 1$, one can easily verify that there exists a family $\left\{F_{a} \mid a \in A\right\}$ uniformly bounded in $C^{\infty}$, such that $F_{a}(0)=0$ and

$$
\partial_{t} \psi=F_{a}(\Xi),
$$

with $\Xi:=\left(v, \psi, \nabla \psi, \operatorname{div} v, \nabla p, \varepsilon \mu \nabla^{2} v, \kappa \nabla^{2} \theta\right)$.

Using the Moser-type estimate (5.9), we find

$$
\left\|\partial_{t} \psi\right\|_{H_{\nu}^{s}} \leqslant C\left(\|\Xi\|_{H^{s-1}}\right)\left\{1+\nu\|\Xi\|_{H^{s}}\right\} .
$$

To complete the proof note that, directly from the definitions (5.22) and the assumption $(\mu, \kappa) \in[0,1]^{2}$, one has $\|\Xi\|_{H^{s-1}} \leqslant R$ and $\nu\|\Xi\|_{H^{s}} \leqslant$ $R+R^{\prime}$.

We are now prepared to prove Proposition 5.1 . 
5.3. Proof of Proposition [5.1, Set $\nu:=\sqrt{\mu+\kappa} \leqslant \sqrt{2}$ and $(\widetilde{p}, \widetilde{v}, \widetilde{\theta}):=$ $\left(\mathcal{Q}_{\varepsilon \nu} p, \mathcal{Q}_{\varepsilon \nu} v, \mathcal{Q}_{\varepsilon \nu} \theta\right)$. We first show that $\widetilde{U}:=(\widetilde{p}, \widetilde{v}, \widetilde{\theta})$ satisfies (4.4) for suitable source terms $f_{1}, f_{2}, f_{3}$ and coefficients $\beta_{1}, \beta_{2}, \beta_{2}^{\sharp}, \beta_{3}$ and $G$. It readily follows from Notation $[5.9$ that $(\widetilde{p}, \widetilde{v}, \widetilde{\theta})$ solves

$$
\left\{\begin{array}{l}
g_{1}(\phi)\left(\partial_{t} \widetilde{p}+v \cdot \nabla \widetilde{p}\right)+\frac{1}{\varepsilon} \operatorname{div} \widetilde{v}-\frac{\kappa}{\varepsilon} B_{1}(\phi) \widetilde{\theta}=f_{1, \mathrm{HF}}^{a, \nu}(U), \\
g_{2}(\phi)\left(\partial_{t} \widetilde{v}+v \cdot \nabla \widetilde{v}\right)+\frac{1}{\varepsilon} \nabla \widetilde{p}-\mu B_{2}(\phi) \widetilde{v}=f_{2, \mathrm{HF}}^{a, \nu}(U), \\
g_{3}(\phi)\left(\partial_{t} \widetilde{\theta}+v \cdot \nabla \widetilde{\theta}+\widetilde{v} \cdot \nabla \theta\right)+\operatorname{div} \widetilde{v}-\kappa B_{3}(\phi) \widetilde{\theta}=f_{3, \mathrm{HF}}^{a, \nu}(U),
\end{array}\right.
$$

where $\phi=(\theta, \varepsilon p)$. Set $\beta_{1}:=\chi_{1} \beta, \beta_{2}:=\chi_{2} \zeta, \beta_{2}^{\sharp}:=\chi_{2} \eta, \beta_{3}:=\chi_{3} \beta$ and $G(\phi, \nabla \phi)=g_{3}(\phi) \nabla \theta$, to obtain that $(\widetilde{p}, \widetilde{v}, \widetilde{\theta})$ satisfies (4.4) where

$$
\begin{aligned}
& f_{1}:=f_{1, \mathrm{HF}}^{a, \nu}(U)+f_{1, \mathrm{HF}}^{\prime}+\mathcal{Q}_{\varepsilon \nu} \Upsilon_{1} \quad \text { with } f_{1, \mathrm{HF}}^{\prime}:=-\frac{\kappa}{\varepsilon} \nabla \chi_{1}(\varepsilon p) \cdot(\beta(\theta) \nabla \widetilde{\theta}), \\
& f_{2}:=f_{2, \mathrm{HF}}^{a, \nu}(U)+f_{2, \mathrm{HF}}^{\prime} \quad \text { with } f_{2, \mathrm{HF}}^{\prime}:=\mu \chi_{2}(\varepsilon p)\{2 D \widetilde{v} \nabla \zeta(\theta)+\operatorname{div} \widetilde{v} \nabla \eta(\theta)\}, \\
& f_{3}:=f_{3, \mathrm{HF}}^{a, \nu}(U)+f_{3, \mathrm{HF}}^{\prime}+\varepsilon \mathcal{Q}_{\varepsilon \nu} \Upsilon_{3} \quad \text { with } f_{3, \mathrm{HF}}^{\prime}:=\kappa \chi_{3}(\varepsilon p) \nabla \beta(\theta) \cdot \nabla \widetilde{\theta},
\end{aligned}
$$

where $\Upsilon_{i}$ are as in system (2.1).

By definition of the norm $\|\cdot\|_{\mathcal{H}_{a}^{s}(T)}$ (see Definition 2.4), the Sobolev embedding Theorem and Lemma [5.14] give

$$
\left\|\left(\phi, \partial_{t} \phi, \nabla \phi, \nu \nabla^{2} \phi, v, \nabla v\right)\right\|_{L^{\infty}([0, T] \times \mathbb{D})} \leqslant C(\Omega),
$$

where $\Omega:=\|U\|_{\mathcal{H}_{a}^{s}(T)}$. Similarly one has $\|\phi(0)\|_{L^{\infty}(\mathbb{D})} \leqslant \Omega_{0}:=\|U(0)\|_{\mathcal{H}_{a, 0}^{s}}$.

Observe that Assumption 2.1 implies that the conditions in Assumption 4.1 are satisfied.

With these preliminary remarks in hands, Theorem 4.5 yields

$$
\|\widetilde{U}\|_{\mathcal{H}_{a}^{0}(T)} \leqslant C\left(\Omega_{0}\right) e^{T C(\Omega)}\|\widetilde{U}(0)\|_{\mathcal{H}_{a, 0}^{0}}+C(\Omega)\left\{\mathfrak{F}(T)+\mathfrak{F}^{\prime}(T)+\mathfrak{F}^{\prime \prime}(T)\right\},
$$

with

$$
\begin{aligned}
\mathfrak{F}(T) & :=\left\|\left(f_{1, \mathrm{HF}}^{a, \nu}(U), f_{2, \mathrm{HF}}^{a, \nu}(U)\right)\right\|_{L^{1}\left(0, T ; H_{\varepsilon \nu}^{1}\right)}+\left\|f_{3, \mathrm{HF}}^{a, \nu}(U)\right\|_{L^{1}\left(0, T ; H_{\nu}^{1}\right)}, \\
\mathfrak{F}^{\prime}(T) & :=\left\|\left(f_{1, \mathrm{HF}}^{\prime}, f_{2, \mathrm{HF}}^{\prime}\right)\right\|_{L^{1}\left(0, T ; H_{\varepsilon \nu}^{1}\right)}+\left\|f_{3, \mathrm{HF}}^{\prime}\right\|_{L^{1}\left(0, T ; H_{\nu}^{1}\right)}, \\
\mathfrak{F}^{\prime \prime}(T) & :=\left\|\mathcal{Q}_{\varepsilon \nu} \Upsilon_{1}\right\|_{L^{1}\left(0, T ; H_{\varepsilon \nu}^{1}\right)}+\left\|\varepsilon \mathcal{Q}_{\varepsilon \nu} \Upsilon_{3}\right\|_{L^{1}\left(0, T ; H_{\nu}^{1}\right)} .
\end{aligned}
$$

One has $\|\widetilde{U}\|_{\mathcal{H}_{a}^{0}(T)}=\left\|\left(I-J_{\varepsilon \nu}\right) U\right\|_{\mathcal{H}_{a}^{s}(T)}$ and similarly $\|\widetilde{U}(0)\|_{\mathcal{H}_{a, 0}^{0}}=$ $\left\|\left(I-J_{\varepsilon \nu}\right) U(0)\right\|_{\mathcal{H}_{a, 0}^{s}} \leqslant \Omega_{0}$. Therefore, in view of the elementary inequality $x+y \leqslant 2 x e^{y}$ (for all $x \geqslant 1$ and $y \geqslant 0$ ), the proof of Proposition 5.1 reduces to establishing that $\mathfrak{F}(T)+\mathfrak{F}^{\prime}(T) \leqslant \sqrt{T} C(\Omega)$.

The estimate $\mathfrak{F}(T) \leqslant \sqrt{T} C(\Omega)$ immediately follows from the preliminaries. Indeed, define $R$ and $R^{\prime}$ as in (5.22). By definition of $\nu:=\sqrt{\mu+\kappa}$, one has $\mu \leqslant \sqrt{\mu} \nu$ and $\kappa \leqslant \sqrt{\kappa} \nu$. Hence, it is easily verified that Lemma 5.11] 
and Lemma 5.14 imply

$$
\left\|f_{1, \mathrm{HF}}^{a, \nu}(U)\right\|_{H_{\varepsilon \nu}^{1}}+\left\|f_{2, \mathrm{HF}}^{a, \nu}(U)\right\|_{H_{\varepsilon \nu}^{1}}+\left\|f_{3, \mathrm{HF}}^{a, \nu}(U)\right\|_{H_{\nu}^{1}} \leqslant C(R)\left\{1+R^{\prime}\right\} .
$$

Since $C(\cdot)$ is non-decreasing, integrating and using the Cauchy-Schwarz estimate, there results

$$
\mathfrak{F}(T) \leqslant \sqrt{T} C\left(\|R\|_{L^{\infty}(0, T)}\right)\left\{1+\left\|R^{\prime}\right\|_{L^{2}(0, T)}\right\} .
$$

This in turn implies the desired estimate $\mathfrak{F}(T) \leqslant \sqrt{T} C(\Omega)$ since, by definition, one has $\Omega \approx\|R\|_{L^{\infty}(0, T)}+\left\|R^{\prime}\right\|_{L^{2}(0, T)}$.

Let us prove that, similarly, $\mathfrak{F}^{\prime}(T) \leqslant \sqrt{T} C(\Omega)$. To do that it is sufficient to prove that (5.24) holds true with $f_{i, \mathrm{HF}}^{a, \nu}(U)$ replaced by $f_{i, \mathrm{HF}}^{\prime}$. This in turn follows from direct estimates. Indeed, observe that

$$
f_{1, \mathrm{HF}}^{\prime}=\kappa \mathcal{F}_{1}(\phi, \nabla p) \nabla \widetilde{\theta}, \quad f_{2, \mathrm{HF}}^{\prime}=\mu \mathcal{F}_{2}(\phi, \nabla \theta) \nabla \widetilde{v}, \quad f_{3, \mathrm{HF}}^{\prime}=\kappa \mathcal{F}_{3}(\phi, \nabla \theta) \nabla \widetilde{\theta},
$$

for some $C^{\infty}$ functions $\mathcal{F}_{i}$ vanishing at the origin. As already seen, one can give estimates for the coefficients $\mathcal{F}_{i}$ by combining the Sobolev embedding Theorem with the Moser estimate (5.7). It is found that

$$
\left\|\left(f_{1, \mathrm{HF}}^{\prime}, f_{2, \mathrm{HF}}^{\prime}\right)\right\|_{H_{\varepsilon \nu}^{1}}+\left\|f_{3, \mathrm{HF}}^{\prime}\right\|_{H_{\nu}^{1}} \leqslant C(R)\left\{\|\sqrt{\kappa} \nabla \widetilde{\theta}\|_{H_{\nu}^{1}}+\|\sqrt{\mu} \nabla \widetilde{v}\|_{H_{\varepsilon \nu}^{1}}\right\} .
$$

We next use $\mathcal{Q}_{\varepsilon \nu} \leqslant \Lambda^{s}$, to obtain

$$
\|\sqrt{\kappa} \nabla \widetilde{\theta}\|_{H_{\nu}^{1}}+\|\sqrt{\mu} \nabla \widetilde{v}\|_{H_{\varepsilon \nu}^{1}} \leqslant \sqrt{\kappa}\|\theta\|_{H_{\nu}^{s+2}}+\sqrt{\mu}\|v\|_{H_{\varepsilon \nu}^{s+2}} \leqslant R^{\prime} .
$$

Consequently, the left-hand side of (5.25) is controlled by $C(R) R^{\prime}$.

To conclude the proof it remains to show that $\mathfrak{F}^{\prime \prime}(T) \leqslant \sqrt{T} C(\Omega)$. This is nothing new in that it follows from the estimates:

$$
\begin{aligned}
\mathfrak{F}^{\prime \prime}(T) & \lesssim \sqrt{T}\left\|\left(\Upsilon_{1}, \Upsilon_{3}\right)\right\|_{L^{2}\left(0, T ; H_{\varepsilon \nu}^{s+1}\right)} \\
& \leqslant \sqrt{T} C\left(\|(\phi, \sqrt{\mu} \nabla v)\|_{L^{\infty}([0, T] \times \mathbb{D})}\right)\left\{1+\sqrt{\mu}\|\nabla v\|_{L^{2}\left(0, T ; H_{\varepsilon \nu}^{s+1}\right)}\right\} \\
& \leqslant \sqrt{T} C\left(\|R\|_{L^{\infty}(0, T)}\right)\left\{1+\left\|R^{\prime}\right\|_{L^{2}(0, T)}\right\} \\
& \leqslant \sqrt{T} C(\Omega) .
\end{aligned}
$$

We have proved Proposition 5.1. This completes the analysis of the high frequency regime.

\section{LOW FREQUENCY REGIME}

This section is devoted to the proof of a priori estimates in the low frequency region, which is the most delicate part.

Proposition 6.1. Given an integer $s>1+d / 2$, there exists a continuous non-decreasing function $C$ such that for all $a=(\varepsilon, \mu, \kappa) \in A$, all $T \in[0,1]$ and all $U=(p, v, \theta) \in C^{1}\left([0, T] ; H^{\infty}(\mathbb{D})\right)$ satisfying (2.1),

$$
\left\|J_{\varepsilon \nu} U\right\|_{\mathcal{H}_{a}^{s}(T)} \leqslant C\left(\Omega_{0}\right) e^{(\sqrt{T}+\varepsilon) C(\Omega)},
$$

where $\nu:=\sqrt{\mu+\kappa}, \Omega_{0}:=\|U(0)\|_{\mathcal{H}_{a, 0}^{s}}, \Omega:=\|U\|_{\mathcal{H}_{a}^{s}(T)}$ (see Definition 2.4 $)$. 
As alluded to previously, the nonlinear energy estimates cannot be obtained from the $L^{2}$ estimates by an elementary argument using differentiation of the equations with respect to spatial derivatives. For such problems a general strategy can be used. One first applies to the equations some operators based on $\left(\varepsilon \partial_{t}\right)$. Next, one uses the special structure of the equations to estimate the spatial derivatives.

This basic strategy has many roots, at least for hyperbolic problems (see, e.g., 1, 18, 39, 38 ). For our purposes, the key point is that the hyperbolic behavior prevails in the low frequency regime. Yet, in sharp contrast with the Euler equations $(\mu=\kappa=0)$, the form of the equations (2.1) shows that the time derivative and the spatial derivatives have not the same weight.

In particular, our analysis requires some preparation. We begin our discussion in 6.1 by establishing some estimates which allows us to commute $J_{\varepsilon \nu}\left(\varepsilon \partial_{t}\right)^{m}$ with the equations. The latter task is achieved in $\$ 6.3$

With these preliminaries established, we can proceed to give an estimate for $J_{\varepsilon \nu}\left(\varepsilon \partial_{t}\right)^{s} U$. The fast components $J_{\varepsilon \nu}(\operatorname{div} v, \nabla p)$ are estimated next by using an induction argument. To conclude, we give the estimates for the slow components $\theta$ and $\operatorname{curl} v$.

For the sake of notational clarity, in this section we deliberately omit the terms $\Upsilon_{1}$ and $\varepsilon \Upsilon_{3}$ in the system (2.1). Nothing is changed in the statements of the results, nor in their proofs.

6.1. Non-isotropic estimates. The fact that the time derivative and the spatial derivatives have not the same weight is made precise by the following lemma (whose easy proof is left to the reader).

Lemma 6.2. There is family $\left\{B_{a, \alpha}\left|a \in A, \alpha \in \mathbb{N}^{d}, 1 \leqslant\right| \alpha \mid \leqslant 2\right\}$ uniformly bounded in $C^{\infty}\left(\mathbb{R}^{N} ; \mathbb{R}^{N \times N}\right.$ ) (where $N=(d+2)^{2}$ ) such that for all $a \in A$ and all smooth solution $(p, v, \theta)$ of (2.1), the function $\Psi$ defined by

$$
\Psi:=\left(\psi, \partial_{t} \psi, \nabla \psi\right) \quad \text { where } \quad \psi:=(\theta, \varepsilon p, \varepsilon v),
$$

solves

$$
\varepsilon \partial_{t} \Psi=\sum_{1 \leqslant j \leqslant d} B_{a, j}(\Psi) \partial_{j} \Psi+\varepsilon(\mu+\kappa) \sum_{1 \leqslant j, k \leqslant d} \partial_{j}\left(B_{a, j k}(\Psi) \partial_{k} \Psi\right) .
$$

We want to introduce an operator based on $\left(\varepsilon \partial_{t}\right)$ which has the weight of a spatial derivative. The previous result suggests introducing the following family of operators.

Definition 6.3. For all $\varepsilon \geqslant 0, \nu \geqslant 0$ and $\ell \in \mathbb{N}$, define the operators

$$
Z_{\varepsilon, \nu}^{\ell}:=\Lambda_{\varepsilon \nu}^{-\ell}\left(\varepsilon \partial_{t}\right)^{\ell}
$$

where, recall from (3.1), that $\Lambda_{\varepsilon \nu}^{-\ell}:=\left(I-(\varepsilon \nu)^{2} \Delta\right)^{-\ell / 2}$.

We will need the following technical ingredient. 
Lemma 6.4. Given $F \in C^{\infty}\left(\mathbb{R}^{n}\right)$ satisfying $F(0)=0$ and $\sigma_{0}>d / 2$, there exists a function $C(\cdot)$ such that for all $\varepsilon \in[0,1]$, all $\nu \in[0,2]$, all $T>0$, all vector-valued function $U \in C^{\infty}\left([0, T] ; H^{\infty}(\mathbb{D})\right)$ and all $\mathbb{N} \ni m \leqslant \sigma_{0}$,

$$
\left\|Z_{\varepsilon, \nu}^{m} F(U)\right\|_{H^{\sigma_{0}-m}} \leqslant C\left(\sum_{0 \leqslant \ell \leqslant m}\left\|Z_{\varepsilon, \nu}^{\ell} U\right\|_{H^{\sigma_{0}-\ell}}\right) .
$$

Proof. To prove this claim, observe that $\left(\varepsilon \partial_{t}\right)^{m} F(U)$ is a sum of terms of the form

$$
f(U)\left(\varepsilon \partial_{t}\right)^{\ell_{1}} u_{1} \cdots\left(\varepsilon \partial_{t}\right)^{\ell_{p}} u_{p},
$$

with $p \leqslant m$ and $\sum_{1 \leqslant i \leqslant p} \ell_{i}=m$. In this formula, $f$ is a $C^{\infty}$ function and $u_{1}, \ldots, u_{p}$ denote coefficients of $U$.

In order to estimate these terms, we use the following result (whose proof follows from Proposition 3.2 by induction):

Let $\alpha=\left(\alpha_{0}, \ldots, \alpha_{p}\right) \in \mathbb{N}^{p+1}$ be such that $\sum_{i=0}^{p} \alpha_{i}=:|\alpha| \leqslant \sigma_{0}$, then

$$
\left\|\Lambda_{\varepsilon \nu}^{-|\alpha|} \prod_{i=0}^{p} V_{i}\right\|_{H^{\sigma_{0}-|\alpha|}} \lesssim \prod_{i=0}^{p}\left\|\Lambda_{\varepsilon \nu}^{-\alpha_{i}} V_{i}\right\|_{H^{\sigma_{0}-\alpha_{i}}},
$$

where the implicit constant depends only on $d, \sigma_{0}$ and $\alpha$.

Set $\tilde{f}:=f-f(0)$. We apply the previous result with $\alpha_{0}=0, \alpha_{i}=\ell_{i}$ $(i \geqslant 1), V_{0}=\tilde{f}(U)$ and $V_{i}=\left(\varepsilon \partial_{t}\right)^{\ell_{i}} u_{i}(i \geqslant 1)$. This yields

$$
\begin{aligned}
& \left\|\Lambda_{\varepsilon \nu}^{-m}\left(f(U)\left(\varepsilon \partial_{t}\right)^{\ell_{1}} u_{1} \cdots\left(\varepsilon \partial_{t}\right)^{\ell_{p}} u_{p}\right)\right\|_{H^{\sigma_{0}-m}} \\
& \quad \lesssim\left(1+\|\widetilde{f}(U)\|_{H^{\sigma_{0}}}\right)\left\|Z_{\varepsilon, \nu}^{\ell_{1}} u_{1}\right\|_{H^{\sigma_{0}-\ell_{1}}} \cdots\left\|Z_{\varepsilon, \nu}^{\ell_{p}} u_{p}\right\|_{H^{\sigma_{0}-\ell_{p}}} .
\end{aligned}
$$

Since $Z_{\varepsilon, \nu}^{0}=I$, the estimate (15.8) implies $\|\widetilde{f}(U)\|_{H^{\sigma_{0}}} \leqslant C\left(\left\|Z_{\varepsilon, \nu}^{0} U\right\|_{H^{\sigma_{0}}}\right)$. Which completes the proof.

Now we are in position to prove that $Z_{\varepsilon, \nu}^{1}$ has the weight of a spatial derivative. The following result states that, for $m \in \mathbb{N}, Z_{\varepsilon, \nu}^{m} \Psi$ satisfies the same estimates as $\Lambda^{m} F(\Psi)$ does (where $F$ is a given function).

Proposition 6.5. Let $s>1+d / 2$ be an integer. There exists a function $C(\cdot)$ such that for all $a=(\varepsilon, \mu, \kappa) \in A$, all $T>0$ and all smooth solution $(p, v, \theta) \in C^{\infty}\left([0, T] ; H^{\infty}(\mathbb{D})\right)$ of (2.1) , if $\nu \in[\mu+\kappa, 2]$ then the function $\Psi$ defined by (6.2) satisfies

$$
\begin{gathered}
\sum_{0 \leqslant \ell \leqslant s}\left\|Z_{\varepsilon, \nu}^{\ell} \Psi\right\|_{H^{s-\ell-1}} \leqslant C\left(\|\Psi\|_{H^{s-1}}\right), \\
\sum_{0 \leqslant \ell \leqslant s}\left\|Z_{\varepsilon, \nu}^{\ell} \Psi\right\|_{H_{\nu}^{s-\ell}} \leqslant C\left(\|\Psi\|_{H^{s-1}}\right)\|\Psi\|_{H_{\nu}^{s}} .
\end{gathered}
$$


Proof. We prove by induction on $m \in\{0, \ldots, s\}$ that

$$
\begin{gathered}
\sum_{0 \leqslant \ell \leqslant m}\left\|Z_{\varepsilon, \nu}^{\ell} \Psi\right\|_{H^{s-\ell-1}} \leqslant C\left(\|\Psi\|_{H^{s-1}}\right), \\
\sum_{0 \leqslant \ell \leqslant m}\left\|Z_{\varepsilon, \nu}^{\ell} \Psi\right\|_{H^{s-\ell}} \leqslant C\left(\|\Psi\|_{H^{s-1}}\right)\|\Psi\|_{H^{s}} .
\end{gathered}
$$

Note that these results are obvious with $m=0$.

Assume the results (6.7)-6.7) at order $m<s$. By definition, one has $Z_{\varepsilon, \nu}^{m+1}=\Lambda_{\varepsilon \nu}^{-1} Z_{\varepsilon, \nu}^{m}\left(\varepsilon \partial_{t}\right)$. It thus follows from (6.3) that

$Z_{\varepsilon, \nu}^{m+1} \Psi=\sum_{j, k} \Lambda_{\varepsilon \nu}^{-1} Z_{\varepsilon, \nu}^{m}\left(B_{a, j}(\Psi) \partial_{j} \Psi\right)+\left(\varepsilon(\mu+\kappa) \partial_{j} \Lambda_{\varepsilon \nu}^{-1}\right) Z_{\varepsilon, \nu}^{m}\left(B_{a, j k}(\Psi) \partial_{k} \Psi\right)$.

Since $\nu \geqslant \mu+\kappa$ one has $\varepsilon(\mu+\kappa) \partial_{j} \Lambda_{\varepsilon \nu}^{-1} \lesssim I$ (see (3.4)). Similarly one has $\Lambda_{\varepsilon \nu}^{-1} \lesssim I$. The proof thus reduces to estimating terms having the form $Z_{\varepsilon, \nu}^{m}\left(B(\Psi) \partial_{j} \Psi\right)$. More precisely, it is sufficient to prove that, for all integer $m<s$ and for all smooth function $B$, one has

$$
\begin{aligned}
& \left\|Z_{\varepsilon, \nu}^{m}\left(B(\Psi) \partial_{j} \Psi\right)\right\|_{H^{s-m-2}} \leqslant C\left(\|\Psi\|_{H^{s-1}}\right), \\
& \left\|Z_{\varepsilon, \nu}^{m}\left(B(\Psi) \partial_{j} \Psi\right)\right\|_{H^{s-m-1}} \leqslant C\left(\|\Psi\|_{H^{s-1}}\right)\|\Psi\|_{H^{s}},
\end{aligned}
$$

where $C(\cdot)$ depends only on a finite number of semi-norms of $B$ in $C^{\infty}$.

Firstly, writing $B(\Psi) \partial_{j} \Psi$ as $\partial_{j} F(\Psi)$ for some $C^{\infty}$ function $F$ such that $F(0)=0$, and using Lemma 6.4 with $\sigma_{0}=s-1$, we find

$$
\left\|Z_{\varepsilon, \nu}^{m}\left(B(\Psi) \partial_{j} \Psi\right)\right\|_{H^{s-m-2}} \lesssim\left\|Z_{\varepsilon, \nu}^{m} F(\Psi)\right\|_{H^{s-1-m}} \leqslant C\left(\sum_{0 \leqslant \ell \leqslant m}\left\|Z_{\varepsilon, \nu}^{\ell} \Psi\right\|_{H^{s-1-\ell}}\right) .
$$

As a consequence, the estimate (6.9) follows from the induction hypothesis (6.7). Moving to the proof of (6.10), we begin with the Leibniz rule

$$
\left(\varepsilon \partial_{t}\right)^{m}\left(B(\Psi) \partial_{j} \Psi\right)=\sum_{0 \leqslant \ell \leqslant m}\left(\begin{array}{c}
m \\
\ell
\end{array}\right)\left(\varepsilon \partial_{t}\right)^{m-\ell} B(\Psi)\left(\varepsilon \partial_{t}\right)^{\ell} \partial_{j} \Psi .
$$

Let $\mathbb{N} \ni \ell \leqslant m$. Since $s>1+d / 2$ and $m \leqslant s-1$, Proposition 3.2 applies with $\sigma_{0}=s-1, \sigma_{1}=m_{1}=m-\ell$ and $\sigma_{2}=m_{2}=\ell$. It yields

$$
\left\|Z_{\varepsilon, \nu}^{m}\left(B(\Psi) \partial_{j} \Psi\right)\right\|_{H^{s-m-1}} \lesssim \sum_{0 \leqslant \ell \leqslant m}\left\|Z_{\varepsilon, \nu}^{m-\ell} B(\Psi)\right\|_{H^{s-1-(m-\ell)}}\left\|Z_{\varepsilon, \nu}^{\ell} \partial_{j} \Psi\right\|_{H^{s-1-\ell}} .
$$

Using Lemma 6.4 to estimate the first term in the summand, we get

$$
\left\|Z_{\varepsilon, \nu}^{m}\left(B(\Psi) \partial_{j} \Psi\right)\right\|_{H^{s-m-1}} \leqslant C\left(\sum_{0 \leqslant \ell \leqslant m}\left\|Z_{\varepsilon, \nu}^{\ell} \Psi\right\|_{H^{s-\ell-1}}\right) \sum_{0 \leqslant \ell \leqslant m}\left\|Z_{\varepsilon, \nu}^{\ell} \Psi\right\|_{H^{s-\ell}} .
$$

Using the induction hypotheses (6.7)-6.7), we prove (6.10).

We next prove a commutator estimate with gain of a factor $\varepsilon$. This technical ingredient is an analogue of the commutator estimate (5.2) [with $\varrho=h]$ we used in the analysis of the high frequency regime. 
Lemma 6.6. Given $s>1+d / 2$, there exists a constant $K$ such that for all $\varepsilon \in[0,1]$, all $\nu \in[0,2]$, all $T>0$, all $m \in \mathbb{N}$ such that $1 \leqslant m \leqslant s$ and all $f, u \in C^{\infty}\left([0, T] ; H^{\infty}(\mathbb{D})\right)$,

$$
\leqslant K \varepsilon\left\{\|f\|_{H^{s}}+\sum_{\ell=0}^{m-1}\left\|Z_{\varepsilon, \nu}^{\ell} \partial_{t} f\right\|_{H^{s-1-\ell}}\right\}\left\{\left\|Z_{\varepsilon, \nu}^{m} u\right\|_{H_{\nu}^{s-m}}+\sum_{\ell=0}^{m-1}\left\|Z_{\varepsilon, \nu}^{\ell} u\right\|_{H^{s-1-\ell}}\right\} .
$$

Proof. The commutator $\left[f, J_{\varepsilon \nu}\left(\varepsilon \partial_{t}\right)^{m}\right] u$ is expanded to

$$
\left[f, J_{\varepsilon \nu}\right]\left(\varepsilon \partial_{t}\right)^{m} u+J_{\varepsilon \nu}\left[f,\left(\varepsilon \partial_{t}\right)^{m}\right] u .
$$

The proof of (6.11) is based on the tools we developed in Section 3

a) We first claim that

$$
\left\|\left[f, J_{\varepsilon \nu}\right]\left(\varepsilon \partial_{t}\right)^{m} u\right\|_{H_{\varepsilon \nu}^{s-m+1}} \lesssim \varepsilon \nu\|f\|_{H^{s}}\left\|Z_{\varepsilon, \nu}^{m} u\right\|_{H^{s-m}} .
$$

Since $s>1+d / 2$ and $1 \leqslant m \leqslant s$, Proposition 3.11 applies with $\left(h, m, \sigma_{0}, \sigma\right)^{4}$ replaced by $(\varepsilon \nu, 1, s, s-m)$. It yields

$$
\left\|\left[f, J_{\varepsilon \nu}\right]\left(\varepsilon \partial_{t}\right)^{m} u\right\|_{H^{s-m}} \lesssim \varepsilon \nu\|f\|_{H^{s}}\left\|\Lambda_{\varepsilon \nu}^{-(2 s-m)}\left(\varepsilon \partial_{t}\right)^{m} u\right\|_{H^{s-m}} .
$$

Since $m \leqslant s$, there holds $\Lambda_{\varepsilon \nu}^{-(2 s-m)} \leqslant \Lambda_{\varepsilon \nu}^{-m}$. Hence, we have the first half of (6.13), namely:

$$
\left\|\left[f, J_{\varepsilon \nu}\right]\left(\varepsilon \partial_{t}\right)^{m} u\right\|_{H^{s-m}} \lesssim \varepsilon \nu\|f\|_{H^{s}}\left\|Z_{\varepsilon, \nu}^{m} u\right\|_{H^{s-m}} .
$$

The technique for obtaining the second half is similar. We first apply Proposition 3.11 with $m=0$, to obtain

$$
\left\|\left[f, J_{\varepsilon \nu}\right]\left(\varepsilon \partial_{t}\right)^{m} u\right\|_{H^{s-m+1}} \lesssim\|f\|_{H^{s}}\left\|Z_{\varepsilon, \nu}^{m} u\right\|_{H^{s-m}},
$$

and we next multiply (6.15) by $\varepsilon \nu$.

b) Moving to the second term in (6.12), we claim that

$$
\begin{aligned}
\left\|J_{\varepsilon \nu}\left[f,\left(\varepsilon \partial_{t}\right)^{m}\right] u\right\|_{H_{\varepsilon \nu}^{s-m+1}} & \\
& \lesssim \varepsilon \sum_{\ell=0}^{m-1}\left\|Z_{\varepsilon, \nu}^{\ell} \partial_{t} f\right\|_{H^{s-1-\ell}} \sum_{\ell=0}^{m-1}\left\|Z_{\varepsilon, \nu}^{\ell} u\right\|_{H^{s-1-\ell}} .
\end{aligned}
$$

Starting from the Leibniz rule, we get

$$
\left[f,\left(\varepsilon \partial_{t}\right)^{m}\right] u=\varepsilon \sum_{\ell=0}^{m-1}\left(\begin{array}{c}
m \\
\ell+1
\end{array}\right)\left(\left(\varepsilon \partial_{t}\right)^{\ell} \partial_{t} f\right)\left(\varepsilon \partial_{t}\right)^{m-1-\ell} u
$$

Let $0 \leqslant \ell \leqslant m-1$. Since $s>1+d / 2$ and $1 \leqslant m \leqslant s$, Proposition 3.2 applies with $\sigma_{0}=s-1, \sigma_{1}=m_{1}=\ell$ and $\sigma_{2}=m_{2}=m-1-\ell$. It yields

$$
\begin{aligned}
& \left.\| \Lambda_{\varepsilon \nu}^{-m+1}\left(\left(\varepsilon \partial_{t}\right)^{\ell} \partial_{t} f\right)\left(\varepsilon \partial_{t}\right)^{m-1-\ell} u\right) \|_{H^{s-m}} \\
& \quad \lesssim\left\|\Lambda_{\varepsilon \nu}^{-\ell}\left(\varepsilon \partial_{t}\right)^{\ell} \partial_{t} f\right\|_{H^{s-1-\ell}}\left\|\Lambda_{\varepsilon \nu}^{-(m-1-\ell)}\left(\varepsilon \partial_{t}\right)^{m-1-\ell} u\right\|_{H^{s-1-(m-1-\ell)}} .
\end{aligned}
$$

\footnotetext{
${ }^{4}$ Here, $m$ refers to the index used in the statement of Lemma 3.4
} 
By summing over all $0 \leqslant \ell \leqslant m-1$, we obtain from the definition of the operators $Z_{\varepsilon, \nu}$ that $\left\|\Lambda_{\varepsilon \nu}^{-m+1}\left[f,\left(\varepsilon \partial_{t}\right)^{m}\right] u\right\|_{H^{s-m}}$ is estimated by the right side of (6.16). To complete the proof of (6.16), use the elementary estimate

$$
\left\|J_{\varepsilon \nu} u\right\|_{H_{\varepsilon \nu}^{s-m+1}} \lesssim\left\|J_{\varepsilon \nu} u\right\|_{H^{s-m}} \lesssim\left\|\Lambda_{\varepsilon \nu}^{-m+1} u\right\|_{H^{s-m}}
$$

6.2. Notations. Let us pause here to set a few notations we use continually in the sequel, as well as to make some running conventions.

Notations.

From now on, we consider a time $0<T \leqslant 1$, a fixed triple of parameter $a=(\varepsilon, \mu, \kappa) \in A$ and a smooth solution $U=(p, v, \theta) \in C^{\infty}\left([0, T] ; H^{\infty}(\mathbb{D})\right)$ of the system (2.1). The notations $\phi, \psi$ and $\Psi$ are shorthand notations for

$$
\phi:=(\theta, \varepsilon p), \quad \psi:=(\theta, \varepsilon p, \varepsilon v) \quad \text { and } \quad \Psi:=\left(\psi, \partial_{t} \psi, \nabla \psi\right) .
$$

Here and below, $s$ always denotes an integer $s>1+d / 2$. We set

$$
\nu:=\sqrt{\mu+\kappa}, \quad \Omega_{0}:=\|U(0)\|_{\mathcal{H}_{a, 0}^{s}} \quad \text { and } \quad \Omega:=\|U\|_{\mathcal{H}_{a}^{s}(T)},
$$

where the norms are defined in Definition 2.4. As in (5.22), set

$$
\begin{aligned}
R & :=\|(p, v)\|_{H_{\varepsilon \nu}^{s+1}}+\|\theta\|_{H_{\nu}^{s+1}}, \\
R^{\prime} & :=\sqrt{\mu+\kappa}\|(\operatorname{div} v, \nabla p)\|_{H^{s}}+\sqrt{\mu}\|\nabla v\|_{H_{\varepsilon \nu}^{s+1}}+\sqrt{\kappa}\|\nabla \theta\|_{H_{\nu}^{s+1}} .
\end{aligned}
$$

With these notations, one has $\Omega \approx\|R\|_{L^{\infty}(0, T)}+\left\|R^{\prime}\right\|_{L^{2}(0, T)}$.

\section{Conventions.}

To say that a smooth non-decreasing function $C:[0,+\infty) \rightarrow[1,+\infty)$ is generic is to say that $C(\cdot)$ is independent of $T, a$ and $(p, v, \theta)$. Given a generic function $C(\cdot)$, we denote by $\widetilde{C}$ the positive constant

$$
\widetilde{C}:=C\left(\Omega_{0}\right) e^{(\sqrt{T}+\varepsilon) C(\Omega)} .
$$

The factor $\varepsilon$ is of no consequence but makes some arguments work more smoothly. We will often use the simple observation that for all generic function $C(\cdot)$, one has $C\left(\Omega_{0}\right)+(\sqrt{T}+\varepsilon) C(\Omega) \leqslant \widetilde{C}$.

To clarify matters, with these conventions, Proposition 6.1 is formulated concisely in the following way: there exists a generic function $C(\cdot)$ such that $\left\|J_{\varepsilon \nu} U\right\|_{\mathcal{H}_{a}^{\mathrm{s}}(T)} \leqslant \widetilde{C}$.

\subsection{Localization in the low frequency region.}

Definition 6.7. Given $m \in \mathbb{N}$, set

$$
\mathcal{X}^{m}:=J_{\varepsilon \nu}\left(\varepsilon \partial_{t}\right)^{m} .
$$


Notation 6.8. Denote by $f_{\mathrm{LF}}^{m}=\left(f_{1, \mathrm{LF}}^{m}, f_{2, \mathrm{LF}}^{m}, f_{3, \mathrm{LF}}^{m}\right)$ the commutator of the equations (2.1) and the operator $\mathcal{X}^{m}$ :

$$
\begin{aligned}
f_{1, \mathrm{LF}}^{m} & :=\left[g_{1}(\phi), \mathcal{X}^{m}\right] \partial_{t} p+\left[g_{1}(\phi) v, \mathcal{X}^{m}\right] \cdot \nabla p-\frac{\kappa}{\varepsilon}\left[B_{1}(\phi), \mathcal{X}^{m}\right] \theta, \\
f_{2, \mathrm{LF}}^{m} & :=\left[g_{2}(\phi), \mathcal{X}^{m}\right] \partial_{t} v+\left[g_{2}(\phi) v, \mathcal{X}^{m}\right] \cdot \nabla v-\mu\left[B_{2}(\phi), \mathcal{X}^{m}\right] v, \\
f_{3, \mathrm{LF}}^{m} & :=\left[g_{3}(\phi), \mathcal{X}^{m}\right] \partial_{t} \theta+\left[g_{3}(\phi) v, \mathcal{X}^{m}\right] \cdot \nabla \theta-\kappa\left[B_{3}(\phi), \mathcal{X}^{m}\right] \theta .
\end{aligned}
$$

The aim of this paragraph is to estimate $f_{\mathrm{LF}}^{m}$. To begin with, consider the case when $m \geqslant 1$.

Lemma 6.9. There exists a generic function $C(\cdot)$ such that for all $m \in \mathbb{N}$ such that $1 \leqslant m \leqslant s$,

$$
\left\|f_{\mathrm{LF}}^{m}\right\|_{\mathcal{H}_{a, 0}^{s-m}}:=\left\|\left(f_{1, \mathrm{LF}}^{m}, f_{2, \mathrm{LF}}^{m}\right)\right\|_{H_{\varepsilon \nu}^{s-m+1}}+\left\|f_{3, \mathrm{LF}}^{m}\right\|_{H_{\nu}^{s-m+1}} \leqslant C(R)\left\{1+R^{\prime}\right\},
$$

where $R$ and $R^{\prime}$ are as defined in (6.20).

Proof. To emphasize the role of the slow component $\Psi$ (as defined in (6.19)), many bounds are given in terms of:

$$
\gamma:=\|\Psi\|_{H^{s-1}} \quad \text { and } \quad \Gamma:=\|\Psi\|_{H_{\nu}^{s}} .
$$

Directly from Lemma 5.14, one has $\left\|\partial_{t} \psi\right\|_{H^{s-1}} \leqslant C(R)$ and $\left\|\partial_{t} \psi\right\|_{H_{\nu}^{s}} \leqslant$ $C(R)\left\{1+R^{\prime}\right\}$. Furthermore, directly from the definition of $\psi$ (see (6.19)), one has $\|(\psi, \nabla \psi)\|_{H_{\nu}^{s}} \leqslant\|(p, v)\|_{H_{\varepsilon \nu}^{s+1}}+\|\theta\|_{H_{\nu}^{s+1}} \leqslant R$. Hence,

$$
\gamma=\|\Psi\|_{H^{s-1}} \leqslant C(R) \quad \text { and } \quad \Gamma=\|\Psi\|_{H_{\nu}^{s}} \leqslant C(R)\left\{1+R^{\prime}\right\} .
$$

Note that Proposition 6.5 applies since the condition $\nu \geqslant \mu+\kappa$ is fulfilled. STEP 1: Estimate for $f_{1, \mathrm{LF}}^{m} \cdot$ a) We begin by proving

$$
\left\|\left[g_{1}(\phi), \mathcal{X}^{m}\right] \partial_{t} p\right\|_{H_{\varepsilon \nu}^{s-m+1}} \leqslant C(\gamma) \Gamma \text {. }
$$

Starting from Lemma 6.6, we find that the left-hand side is bounded by

$$
\left\{\left\|\tilde{g}_{1}(\phi)\right\|_{H^{s}}+\sum_{\ell=0}^{m-1}\left\|Z_{\varepsilon, \nu}^{\ell} \partial_{t} g_{1}(\phi)\right\|_{H^{s-1-\ell}}\right\}\left\{\sum_{\ell=0}^{m}\left\|Z_{\varepsilon, \nu}^{\ell}\left(\varepsilon \partial_{t} p\right)\right\|_{H_{\nu}^{s-\ell}}\right\} .
$$

Since $\Psi=\left(\ldots,\left(\varepsilon \partial_{t}\right) p, \ldots\right)$, the estimate (6.6) implies that the second factor in (6.25) is estimated by $C(\gamma) \Gamma$. Moving to the first factor, note that $\partial_{t} g_{1}(\phi)=F\left(\phi, \partial_{t} \phi\right)$ for some $C^{\infty}$ function $F$ such that $F(0)=0$. As a consequence, Lemma 6.4 implies

$$
\sum_{\ell=0}^{m-1}\left\|Z_{\varepsilon, \nu}^{\ell} \partial_{t} g_{1}(\phi)\right\|_{H^{s-1-\ell}} \leqslant C\left(\sum_{\ell=0}^{m-1}\left\|Z_{\varepsilon, \nu}^{\ell}\left(\phi, \partial_{t} \phi\right)\right\|_{H^{s-1-\ell}}\right) .
$$

Again, since $\Psi=\left(\phi, \ldots, \partial_{t} \phi, \ldots\right)$, the estimate (6.5) implies that the righthand side in the previous estimate is bounded by $C(\gamma)$. It remains to estimate $\left\|\tilde{g}_{1}(\phi)\right\|_{H^{s}}$. To do so write

$$
\left\|\tilde{g}_{1}(\phi)\right\|_{H^{s}} \leqslant C\left(\|\phi\|_{H^{s}}\right) \leqslant C\left(\|(\psi, \nabla \psi)\|_{H^{s-1}}\right) \leqslant C(\gamma) .
$$


b) We next prove that

$$
\left\|\left[g_{1}(\phi) v, \mathcal{X}^{m}\right] \cdot \nabla p\right\|_{H_{\varepsilon \nu}^{s-m+1}} \leqslant C(\gamma)\left\{\|\nabla p\|_{H^{s-1}}+\Gamma\right\} .
$$

Lemma 6.6 implies that the left-hand side is bounded by

$$
\begin{aligned}
\left\{\left\|\varepsilon g_{1}(\phi) v\right\|_{H^{s}}\right. & \left.+\sum_{\ell=0}^{m-1}\left\|Z_{\varepsilon, \nu}^{\ell} \partial_{t}\left(\varepsilon g_{1}(\phi) v\right)\right\|_{H^{s-1-\ell}}\right\} \\
& \times\left\{\|\nabla p\|_{H^{s-1}}+\sum_{\ell=1}^{m}\left\|Z_{\varepsilon, \nu}^{\ell} \nabla p\right\|_{H_{\nu}^{s-\ell}}\right\} .
\end{aligned}
$$

By definition $\psi=(\theta, \varepsilon p, \varepsilon v)$. Hence, one can rewrite $\varepsilon g_{1}(\phi) v$ as $G(\psi)$ for some $C^{\infty}$ function $G$. Consequently, the first term in the above written product is the exact analogue of the first term in (6.25) with $g_{1}(\phi)$ replaced by $G(\psi)$. We thus obtain that this term is estimated by $C(\gamma)$.

Next, using the very definitions of $Z_{\varepsilon, \nu}^{\ell}$, write

$$
\sum_{\ell=1}^{m}\left\|Z_{\varepsilon, \nu}^{\ell} \nabla p\right\|_{H_{\nu}^{s-\ell}}=\sum_{\ell=1}^{m}\left\|Z_{\varepsilon, \nu}^{\ell-1} \Lambda_{\varepsilon \nu}^{-1} \nabla\left(\varepsilon \partial_{t} p\right)\right\|_{H_{\nu}^{s-\ell}} .
$$

Since $\Lambda_{\varepsilon \nu}^{-1} \lesssim I$, this in turn implies

$$
\sum_{\ell=1}^{m}\left\|Z_{\varepsilon, \nu}^{\ell} \nabla p\right\|_{H_{\nu}^{s-\ell}} \leqslant \sum_{\ell=0}^{m-1}\left\|Z_{\varepsilon, \nu}^{\ell}\left(\varepsilon \partial_{t} p\right)\right\|_{H_{\nu}^{s-\ell}} .
$$

Which, as in the previous step a), is estimated by $C(\gamma) \Gamma$.

c) To complete the estimate of $f_{1, \mathrm{LF}}^{m}$, we establish

$$
\frac{\kappa}{\varepsilon}\left\|\left[B_{1}(\phi), \mathcal{X}^{m}\right] \theta\right\|_{H_{\varepsilon \nu}^{s-m+1}} \leqslant C(\gamma, R)\left\{\|\kappa \theta\|_{H_{\nu}^{s+2}}+\Gamma\right\} .
$$

Parallel to (5.17), we decompose the commutator $\kappa \varepsilon^{-1}\left[B_{1}(\phi), \mathcal{X}^{m}\right] \theta$ as

$$
\frac{\kappa}{\varepsilon}\left[\mathcal{E}_{1}(\phi), \mathcal{X}^{m}\right] \Delta \theta+\frac{\kappa}{\varepsilon}\left[\mathcal{E}_{2}(\phi, \nabla \phi), \mathcal{X}^{m}\right] \cdot \nabla \theta
$$

where $\mathcal{E}_{1}(\phi):=\chi_{1}(\varepsilon p) \beta(\theta)$ and $\mathcal{E}_{2}(\phi, \nabla \phi):=\chi_{1}(\varepsilon p) \beta^{\prime}(\theta) \nabla \theta$.

Replacing $\partial_{t} p$ with $\kappa \varepsilon^{-1} \Delta \theta$, the arguments given in a) yield

$$
\frac{\kappa}{\varepsilon}\left\|\left[\mathcal{E}_{1}(\phi), \mathcal{X}^{m}\right] \Delta \theta\right\|_{H_{\varepsilon \nu}^{s-m+1}} \leqslant C(\gamma) \sum_{\ell=0}^{m}\left\|Z_{\varepsilon, \nu}^{\ell}(\kappa \Delta \theta)\right\|_{H_{\nu}^{s-\ell}} .
$$

We split the sum in the previous right-hand side as

$$
\|\kappa \Delta \theta\|_{H_{\nu}^{s}}+\sum_{\ell=1}^{m}\left\|Z_{\varepsilon, \nu}^{\ell}(\kappa \Delta \theta)\right\|_{H_{\nu}^{s-\ell}} .
$$

The first term is bounded by $\|\kappa \theta\|_{H_{\nu}^{s+2}}$. As regards the second term, we proceed as in b). Namely, write

$$
\sum_{\ell=1}^{m} \kappa\left\|Z_{\varepsilon, \nu}^{\ell} \Delta \theta\right\|_{H_{\nu}^{s-\ell}}=\sum_{\ell=1}^{m}\left\|Z_{\varepsilon, \nu}^{\ell-1}\left(\varepsilon \kappa \Lambda_{\varepsilon \nu}^{-1} \Delta\right) \partial_{t} \theta\right\|_{H_{\nu}^{s-\ell}} \leqslant \sum_{\ell=0}^{m-1}\left\|Z_{\varepsilon, \nu}^{\ell} \partial_{t} \theta\right\|_{H_{\nu}^{s-\ell}}
$$


where we used $\varepsilon \kappa \Lambda_{\varepsilon \nu}^{-1} \Delta \lesssim \Lambda^{1}$ (which stems from $\kappa \leqslant \sqrt{\kappa} \leqslant \nu$ ). Note that $\Psi=\left(\ldots, \partial_{t} \theta, \ldots\right)$. Therefore, the estimate (6.6) implies that the right-hand side of the previous inequality is controlled by $C(\gamma) \Gamma$.

We now have to estimate the $H_{\varepsilon \nu}^{s-m+1}$-norm of the second term in (6.31). Since $\kappa \leqslant \nu$ and $\kappa \leqslant 1$, Lemma 6.6 implies that this term is bounded by

$$
\left\{\left\|\nu \mathcal{E}_{2}\right\|_{H^{s}}+\sum_{\ell=0}^{m-1}\left\|\nu Z_{\varepsilon, \nu}^{\ell} \partial_{t} \mathcal{E}_{2}\right\|_{H^{s-1-\ell}}\right\}\left\{\|\nabla \theta\|_{H^{s-1}}+\sum_{\ell=1}^{m}\left\|\nu Z_{\varepsilon, \nu}^{\ell} \nabla \theta\right\|_{H^{s-\ell}}\right\} .
$$

This in turn is bounded by $C(\gamma)\{C(R)+\Gamma\}$ since

$$
\begin{array}{lr}
\left\|\nu \mathcal{E}_{2}(\phi, \nabla \phi)\right\|_{H^{s}} \leqslant C(R), \quad \nu\left\|Z_{\varepsilon, \nu}^{\ell} \partial_{t} \mathcal{E}_{2}(\phi, \nabla \phi)\right\|_{H^{s-1-\ell}} \leqslant C(\gamma) \Gamma, \\
\|\nabla \theta\|_{H^{s-1}} \leqslant \gamma, & \forall \ell \in[1, m], \quad\left\|\nu Z_{\varepsilon, \nu}^{\ell} \nabla \theta\right\|_{H^{s-\ell}} \leqslant C(\gamma) .
\end{array}
$$

The first inequality in (6.32) follows from (5.7). The second inequality in (6.32) follows from (6.10) since the term $\partial_{t} \mathcal{E}_{2}(\phi, \nabla \phi)$ can be written $F(\Psi) \nabla \Psi$ for some $C^{\infty}$ function $F$. The first inequality in (6.33) is obvious. In order to prove the last one, as already seen, one can write

$$
\begin{aligned}
\left\|\nu Z_{\varepsilon, \nu}^{\ell} \nabla \theta\right\|_{H^{s-\ell}} & \lesssim\left\|Z_{\varepsilon, \nu}^{\ell-1}\left(\varepsilon \nu \Lambda_{\varepsilon \nu}^{-1} \nabla\right) \partial_{t} \theta\right\|_{H^{s-\ell}} \\
& \lesssim\left\|Z_{\varepsilon, \nu}^{\ell-1} \Psi\right\|_{H^{s-1-(\ell-1)}} \leqslant C(\gamma) .
\end{aligned}
$$

d) By combining (6.24) with (6.27) and (6.30), we obtain

$$
\left\|f_{1, \mathrm{LF}}^{m}\right\|_{H_{\varepsilon \nu}^{s-m+1}} \leqslant C(\gamma, R)\left\{\|\kappa \theta\|_{H_{\nu}^{s+2}}+\Gamma\right\}
$$

so that the desired estimate $\left\|f_{1, \mathrm{LF}}^{m}\right\|_{H_{\varepsilon \nu}^{s-m+1}} \leqslant C(R)\left\{1+R^{\prime}\right\}$ follows from the observation (6.23).

STEP 2: Estimate for $f_{2, \mathrm{LF}}^{m}$. Note that one can obtain $f_{2, \mathrm{LF}}^{m}$ from $f_{1, \mathrm{LF}}^{m}$ by replacing $p$ by $v$ and $\theta$ by $\varepsilon v$. Therefore, we are in the situation of the previous step and hence conclude that

$$
\begin{aligned}
\left\|\left[g_{2}(\phi), \mathcal{X}^{m}\right] \partial_{t} v\right\|_{H_{\varepsilon \nu}^{s-m+1}} & \leqslant C(\gamma) \Gamma \\
\left\|\left[g_{2}(\phi) v, \mathcal{X}^{m}\right] \cdot \nabla v\right\|_{H_{\varepsilon \nu}^{s-m+1}} & \leqslant C(\gamma)\left\{\|\nabla v\|_{H^{s-1}}+\Gamma\right\}, \\
\mu\left\|\left[B_{2}(\phi), \mathcal{X}^{m}\right] v\right\|_{H_{\varepsilon \nu}^{s-m+1}} & \leqslant C(\gamma, R)\left\{\|\varepsilon \mu v\|_{H_{\nu}^{s+2}}+\Gamma\right\} .
\end{aligned}
$$

STEP 3: Estimate for $f_{3, \mathrm{LF}}^{m}$. Note that, for all $u \in H_{\nu}^{\sigma}$,

$$
\|u\|_{H_{\nu}^{\sigma}} \leqslant \varepsilon^{-1}\|u\|_{H_{\varepsilon \nu}^{\sigma}} .
$$

Hence,

$$
\begin{aligned}
\|\left[g_{3}(\phi), \mathcal{X}^{m}\right] & \partial_{t} \theta\left\|_{H_{\nu}^{s-m+1}}+\kappa\right\|\left[B_{3}(\phi), \mathcal{X}^{m}\right] \theta \|_{H_{\nu}^{s-m+1}} \\
& \leqslant \frac{1}{\varepsilon}\left\|\left[g_{3}(\phi), \mathcal{X}^{m}\right] \partial_{t} \theta\right\|_{H_{\varepsilon \nu}^{s-m+1}}+\frac{\kappa}{\varepsilon}\left\|\left[B_{3}(\phi), \mathcal{X}^{m}\right] \theta\right\|_{H_{\varepsilon \nu}^{s-m+1}} .
\end{aligned}
$$

The first term in the right-hand side is estimated as in a) above [replacing $\partial_{t} p$ by $\left.\varepsilon^{1} \partial_{t} \theta\right]$ and the second term has been estimated in $\left.\mathbf{c}\right)$. 
For technical reasons, the estimate for $\left[g_{3}(\phi) v, \mathcal{X}^{m}\right] \cdot \nabla \theta$ is somewhat more complicated. We argue as in the proof of Lemma 6.6. Set $f:=g_{3}(\phi) v$ and $u=\nabla \theta$. We begin by splitting the commutator $\left[f, \mathcal{X}^{m}\right] \cdot u$ as

$$
P+Q:=J_{\varepsilon \nu}\left[f,\left(\varepsilon \partial_{t}\right)^{m}\right] \cdot u+\left[f, J_{\varepsilon \nu}\right] \cdot\left(\varepsilon \partial_{t}\right)^{m} u .
$$

By combining (6.14) with (6.15) multiplied by $\nu$, we find

$$
\|Q\|_{H_{\nu}^{s-m+1}} \lesssim\|f\|_{H^{s}}\left\|Z_{\varepsilon, \nu}^{m} u\right\|_{H_{\nu}^{s-m}} \leqslant C(\gamma, R) \Gamma
$$

where we used (6.33).

Our next task is to show a similar estimate for $P$. To do so we decompose $P$ into two parts:

$$
P_{1}+P_{2}:=\left\{J_{\varepsilon \nu}\left[f,\left(\varepsilon \partial_{t}\right)^{m}\right] \cdot u-J_{\varepsilon \nu}\left(u \cdot\left(\varepsilon \partial_{t}\right)^{m} f\right)\right\}+J_{\varepsilon \nu}\left(u \cdot\left(\varepsilon \partial_{t}\right)^{m} f\right) .
$$

Let us prove that $\left\|P_{1}\right\|_{H_{\nu}^{s-m+1}} \leqslant C(\gamma)$. In light of (6.37), all we need to prove is that

$$
\left\|P_{1}\right\|_{H_{\varepsilon \nu}^{s-m+1}} \leqslant \varepsilon C(\gamma) .
$$

Repeat the proof of Lemma 6.6, to obtain

$$
\left\|P_{1}\right\|_{H_{\varepsilon \nu}^{s-m+1}} \lesssim \varepsilon \sum_{\ell=1}^{m-1}\left\|Z_{\varepsilon, \nu}^{\ell} \partial_{t} f\right\|_{H^{s-1-\ell}}\left\|Z_{\varepsilon, \nu}^{\ell} u\right\|_{H^{s-1-\ell}} .
$$

The sum differs from the one that appears in Lemma 6.6 in that it is indexed by $\ell \geqslant 1$ instead of $\ell \geqslant 0$. This fact allows us to write

$$
\left\|P_{1}\right\|_{H_{\varepsilon \nu}^{s-m+1}} \lesssim \varepsilon \sum_{\ell=1}^{m-1}\left\|Z_{\varepsilon, \nu}^{\ell}\left(\varepsilon \partial_{t}\right) f\right\|_{H^{s-1-\ell}}\left\|Z_{\varepsilon, \nu}^{\ell-1} \partial_{t} u\right\|_{H^{s-1-\ell}} .
$$

Let $1 \leqslant \ell \leqslant m-1$. Write $\left(\varepsilon \partial_{t}\right) f$ as $F(\Psi)$ for some $C^{\infty}$ function $F$ such that $F(0)=0$. By combining Lemma 6.4 and the estimate [6.5), we obtain

$$
\left\|Z_{\varepsilon, \nu}^{\ell}\left(\varepsilon \partial_{t}\right) f\right\|_{H^{s-1-\ell}} \leqslant C(\gamma)
$$

Moving to the estimate of $Z_{\varepsilon, \nu}^{\ell-1} \partial_{t} u$, use the very definitions of $u=\nabla \theta$ and $\Psi=\left(\ldots, \partial_{t} \theta, \ldots\right)$, to obtain thanks to 6.5

$$
\left\|Z_{\varepsilon, \nu}^{\ell-1} \partial_{t} u\right\|_{H^{s-1-\ell}} \leqslant\left\|Z_{\varepsilon, \nu}^{\ell-1} \Psi\right\|_{H^{s-\ell}}=\left\|Z_{\varepsilon, \nu}^{\ell-1} \Psi\right\|_{H^{s-(\ell-1)-1}} \leqslant C(\gamma) .
$$

We have proved (6.39), so to conclude it remains only to estimate the second term $P_{2}$ in (6.38). This is accomplished using

$$
\left\|P_{2}\right\|_{H_{\nu}^{s-m+1}} \leqslant\|u\|_{H_{\nu}^{s}}\left\|Z_{\varepsilon, \nu}^{m-1}\left(\varepsilon \partial_{t}\right) f\right\|_{H_{\nu}^{s-m+1}}
$$

as the reader can verify, yielding the bound $\left\|P_{2}\right\|_{H_{\nu}^{s-m+1}} \leqslant C(\gamma, R) \Gamma$.

This completes the proof of Lemma 6.9

Note that in the case when $m=0$, the previous method does not work as it stands, as the estimate (6.13) is no longer correct.

Now we give an estimate valid for all $0 \leqslant m \leqslant s-1$. 
Lemma 6.10. There exists a generic function $C(\cdot)$ such that for all $m \in \mathbb{N}$ such that $0 \leqslant m \leqslant s-1$,

$$
\left\|f_{\mathrm{LF}}^{m}\right\|_{\mathcal{H}_{a, 0}^{s-1-m}}:=\left\|\left(f_{1, \mathrm{LF}}^{m}, f_{2, \mathrm{LF}}^{m}\right)\right\|_{H_{\varepsilon \nu}^{s-m}}+\left\|f_{3, \mathrm{LF}}^{m}\right\|_{H_{\nu}^{s-m}} \leqslant C(R) .
$$

The estimate (6.40) is nothing new in that it can be deduced by following the proof of Lemma 6.9. One only has to use the following analogue of the calculus inequality in Lemma 6.6

$$
\begin{gathered}
\left\|\left[f, J_{\varepsilon \nu}\left(\varepsilon \partial_{t}\right)^{m}\right] u\right\|_{H_{\varepsilon \nu}^{s-m}} \leqslant \\
K \varepsilon\left\{\|f\|_{H^{s}}+\sum_{\ell=0}^{m-1}\left\|Z_{\varepsilon, \nu}^{\ell} \partial_{t} f\right\|_{H^{s-2-\ell}}\right\}\left\{\left\|Z_{\varepsilon, \nu}^{m} u\right\|_{H^{s-1-m}}+\sum_{\ell=0}^{m-1}\left\|Z_{\varepsilon, \nu}^{\ell} u\right\|_{H^{s-1-\ell}}\right\} .
\end{gathered}
$$

In the case when $m=0$ the sum $\sum_{0}^{-1}$ is interpreted as 0 . The index $s-2-\ell$ in the second term of the first set of parentheses is not a typographical error. It is of use to us for the estimate of $\partial_{t} \mathcal{E}_{2}(\phi, \nabla \phi)$ where $\mathcal{E}_{2}$ is as in (6.32).

6.4. The fast components. We give here the estimates for the fast components $\operatorname{div} v$ and $\nabla p$. We make use of the notations introduced in $\$ 6.2$.

Notation 6.11. For all integer $m \leqslant s$, set $U_{m}:=\mathcal{X}^{m} U$ where recall $\mathcal{X}^{m}=J_{\varepsilon \nu}\left(\varepsilon \partial_{t}\right)^{m}$.

As a preliminary step towards the estimate of $(\operatorname{div} v, \nabla p)$ we estimate the $\mathcal{H}_{a}^{0}(T)$-norm of $U_{m}$.

Lemma 6.12. For all integer $m \leqslant s$, there is a generic function $C$ such that $\left\|U_{m}\right\|_{\mathcal{H}_{a}^{0}(T)} \leqslant \widetilde{C}$, where $\widetilde{C}$ is as defined in (6.21).

Having estimated the commutators $f_{\mathrm{LF}}^{m}$, this result can be deduced by following the end of proof of Proposition 5.1 (see \$5.3). We therefore only indicate the points at which the argument is slightly different.

Proof. It readily follows from Notation 6.8 that $(\widetilde{p}, \widetilde{v}, \widetilde{\theta}):=\left(p_{m}, v_{m}, \theta_{m}\right)$ satisfies the linearized system (4.1) with

$$
\begin{array}{lll}
f_{1}:=f_{1, \mathrm{LF}}^{m}+f_{1, \mathrm{LF}}^{\prime} \quad \text { where } \quad f_{1, \mathrm{LF}}^{\prime}:=-\frac{\kappa}{\varepsilon} \nabla \chi_{1}(\varepsilon p) \cdot\left(\beta(\theta) \nabla \theta_{m}\right), \\
f_{2}:=f_{2, \mathrm{LF}}^{m}+f_{2, \mathrm{LF}}^{\prime} \quad \text { where } \quad f_{2, \mathrm{LF}}^{\prime}:=\mu \chi_{2}(\varepsilon p)\left\{2 D v_{m} \nabla \zeta(\theta)+\operatorname{div} v_{m} \nabla \eta(\theta)\right\}, \\
f_{3}:=f_{3, \mathrm{LF}}^{m}+f_{3, \mathrm{LF}}^{\prime} \quad \text { where } \quad f_{3, \mathrm{LF}}^{\prime}:=\kappa \chi_{3}(\varepsilon p) \nabla \beta(\theta) \cdot \nabla \theta_{m} .
\end{array}
$$

recalling that we deliberately omit the terms $\Upsilon$ and $\varepsilon \Upsilon$ in the system (2.1).

Applying Theorem 4.3, we get

$$
\left\|U_{m}\right\|_{\mathcal{H}_{a}^{0}(T)} \leqslant C\left(\Omega_{0}\right) e^{T C(\Omega)}\left\|U_{m}(0)\right\|_{\mathcal{H}_{a, 0}^{0}}+C(\Omega) \mathfrak{F}(T)+C(\Omega) \mathfrak{F}^{\prime}(T),
$$

with

$$
\begin{aligned}
\mathfrak{F}(T) & :=\left\|\left(f_{1, \mathrm{LF}}^{m}, f_{2, \mathrm{LF}}^{m}\right)\right\|_{L^{1}\left(0, T ; H_{\varepsilon \nu}^{1}\right)}+\left\|f_{3, \mathrm{LF}}^{m}\right\|_{L^{1}\left(0, T ; H_{\nu}^{1}\right)} \\
\mathfrak{F}^{\prime}(T) & :=\left\|\left(f_{1, \mathrm{LF}}^{\prime}, f_{2, \mathrm{LF}}^{\prime}\right)\right\|_{L^{1}\left(0, T ; H_{\varepsilon \nu}^{1}\right)}+\left\|f_{3, \mathrm{LF}}^{\prime}\right\|_{L^{1}\left(0, T ; H_{\nu}^{1}\right)}
\end{aligned}
$$


The proof thus reduces to establishing that $\left\|U_{m}(0)\right\|_{\mathcal{H}_{a, 0}^{0}} \leqslant C\left(\Omega_{0}\right)$ and $\mathfrak{F}(T)+\mathfrak{F}^{\prime}(T) \leqslant \sqrt{T} C(\Omega)$. To fix matters, we concentrate on the hardest case when $m=s$. Note that the conclusion of Lemma 6.9 is the exact analogue of (5.24). Hence, one has $\mathfrak{F}(T) \leqslant \sqrt{T} C(\Omega)$. As in $\$ 5.3$, all that has to be done in order to prove $\mathfrak{F}^{\prime}(T) \leqslant \sqrt{T} C(\Omega)$ is to check $\sqrt{\kappa}\left\|\nabla \theta_{S}\right\|_{H_{\nu}^{1}}+$ $\sqrt{\mu}\left\|\nabla v_{s}\right\|_{H_{\varepsilon \nu}^{1}} \leqslant C(R)\left\{1+R^{\prime}\right\}$. To do so, using the definition of $\theta_{s}=$ $J_{\varepsilon \nu}\left(\varepsilon \partial_{t}\right)^{s} \theta$, we first rewrite $\nabla \theta_{s}$ as $\varepsilon J_{\varepsilon \nu}\left(\varepsilon \partial_{t}\right)^{s-1} \nabla \partial_{t} \theta$. Next, by combining the estimate $\left\|\varepsilon J_{\varepsilon \nu} u\right\|_{H_{\nu}^{1}} \leqslant\left\|J_{\varepsilon \nu} u\right\|_{H_{\varepsilon \nu}^{1}} \lesssim\left\|J_{\varepsilon \nu} u\right\|_{L^{2}}$ with the inequality $\sqrt{\kappa} \leqslant$ $\nu$ and the definition of $\Psi=\left(\ldots, \partial_{t} \theta, \ldots\right)$, we obtain

$$
\sqrt{\kappa}\left\|\nabla \theta_{S}\right\|_{H_{\nu}^{1}} \lesssim \nu\left\|Z_{\varepsilon, \nu}^{s-1} \nabla \Psi\right\|_{L^{2}} \leqslant\left\|Z_{\varepsilon, \nu}^{s-1} \Psi\right\|_{H_{\nu}^{1}} .
$$

Analogous computations lead to

$$
\sqrt{\mu}\left\|\nabla v_{S}\right\|_{H_{\varepsilon \nu}^{1}} \lesssim\left\|v_{S}\right\|_{H_{\nu}^{1}} \lesssim\left\|Z_{\varepsilon, \nu}^{S-1}\left(\varepsilon \partial_{t}\right) v\right\|_{H_{\nu}^{1}} \leqslant\left\|Z_{\varepsilon, \nu}^{S-1} \Psi\right\|_{H_{\nu}^{1}}
$$

Hence, the desired bound follows from (6.6) and (6.23).

The technique for estimating the initial data is similar. Indeed, one has $\left\|U_{S}\right\|_{\mathcal{H}_{a, 0}^{0}} \lesssim\left\|Z_{\varepsilon, \nu}^{s-1} \Psi\right\|_{L^{2}}$, as the reader can verify, yielding the bound $\left\|U_{S}(0)\right\|_{\mathcal{H}_{a, 0}^{0}} \leqslant C(R(0))=C\left(\Omega_{0}\right)$.

We now come to the main estimates of this part.

Notation 6.13. Define $\|u\|_{\mathcal{K}_{\nu}^{\sigma}(T)}:=\|u\|_{L^{\infty}\left(0, T ; H^{\sigma-1}\right)}+\nu\|u\|_{L^{2}\left(0, T ; H^{\sigma}\right)}$.

Lemma 6.14. Let $\widetilde{U}:=(\widetilde{p}, \widetilde{v}, \widetilde{\theta})$ be a solution of the system:

$$
\left\{\begin{array}{l}
g_{1}(\phi) \partial_{t} \widetilde{p}+\frac{1}{\varepsilon} \operatorname{div} \widetilde{v}-\frac{\kappa}{\varepsilon} B_{1}(\phi) \widetilde{\theta}=f_{1}, \\
g_{2}(\phi) \partial_{t} \widetilde{v}+\frac{1}{\varepsilon} \nabla \widetilde{p}-\mu B_{2}(\phi) \widetilde{v}=f_{2}, \\
g_{3}(\phi) \partial_{t} \widetilde{\theta}+\operatorname{div} \widetilde{v}-\kappa B_{3}(\phi) \widetilde{\theta}=f_{3} .
\end{array}\right.
$$

If the Fourier transform of $\widetilde{U}$ is supported in the ball $\{|\xi| \leqslant 2 / \varepsilon \nu\}$, then there exists a generic function $C(\cdot)$ such that for all $\sigma \in[1, s]$,

$$
\begin{aligned}
\|\widetilde{p}\|_{\mathcal{K}_{\nu}^{\sigma+1}(T)}+\|\operatorname{div} \widetilde{v}\|_{\mathcal{K}_{\nu}^{\sigma}(T)} & \\
\leqslant & \widetilde{C}\left\|\left(\varepsilon \partial_{t}\right) \widetilde{p}\right\|_{\mathcal{K}_{\nu}^{\sigma}(T)}+\widetilde{C}\left\|\left(\varepsilon \partial_{t}\right) \operatorname{div} \widetilde{v}\right\|_{\mathcal{K}_{\nu}^{\sigma-1}(T)} \\
& +\widetilde{C}\|\widetilde{p}\|_{L^{\infty}\left(0, T ; L^{2}\right)}+\widetilde{C}\|\widetilde{\theta}(0)\|_{H_{\nu}^{\sigma+1}}+\varepsilon C(\Omega)\|\mu \widetilde{v}\|_{\mathcal{K}_{\nu}^{\sigma+1}(T)} \\
& +\varepsilon C(\Omega)\left\|\left(f_{1}, f_{2}\right)\right\|_{\mathcal{K}_{\nu}^{\sigma}(T)}+\nu \widetilde{C}\left\|f_{3}\right\|_{L^{2}\left(0, T ; H^{\sigma}\right)} .
\end{aligned}
$$

Remark 6.15. This result is an elaboration of Lemma 2.10 in 1 .

Proof. a) For further references, we first prove three estimates.

1) Let $\sigma_{0}>d / 2$ and $\sigma \in\left[0, \sigma_{0}\right]$. There exists a constant $K$ such that for all $\varrho \geqslant 0$ and for all $\left(u_{1}, u_{2}\right) \in H^{\sigma_{0}} \times H_{\varrho}^{\sigma}(\mathbb{D})$,

$$
\left\|u_{1} u_{2}\right\|_{H_{\varrho}^{\sigma}} \leqslant K\left\|u_{1}\right\|_{H^{\sigma_{0}}}\left\|u_{2}\right\|_{H_{\varrho}^{\sigma}} .
$$


To prove this result, we use a standard Moser's estimate. Since $\sigma_{0}>d / 2$, the product maps continuously $H^{\sigma_{0}} \times H^{r}$ to $H^{r}$ for all $r \in\left[-\sigma_{0}, \sigma_{0}\right]$. Hence, one has

$$
\begin{aligned}
\left\|u_{1} u_{2}\right\|_{H_{\varrho}^{\sigma}} & :=\left\|u_{1} u_{2}\right\|_{H^{\sigma-1}}+\varrho\left\|u_{1} u_{2}\right\|_{H^{\sigma}} \\
& \lesssim\left\|u_{1}\right\|_{H^{\sigma_{0}}}\left(\left\|u_{2}\right\|_{H^{\sigma-1}}+\varrho\left\|u_{2}\right\|_{H^{\sigma}}\right)=\left\|u_{1}\right\|_{H^{\sigma_{0}}}\left\|u_{2}\right\|_{H_{\varrho}^{\sigma}} .
\end{aligned}
$$

2) Let $\sigma \in[0, s]$ and $q \in[1,+\infty]$. Given a $C^{\infty}$ function $F$, there exists a generic function $C$ such that for all $\varrho \geqslant 0$, and for all $u \in L^{q}\left(0, T ; H_{\varrho}^{\sigma}\right)$,

$$
\|F(\psi) u\|_{L^{q}\left(0, T ; H_{\varrho}^{\sigma}\right)} \lesssim \widetilde{C}\|u\|_{L^{q}\left(0, T ; H_{\varrho}^{\sigma}\right)},
$$

where $\psi$ is as defined in (6.19).

In light of (6.43), to prove this estimate we need only show that

$$
\|F(\psi)\|_{L^{\infty}\left(0, T ; H^{s}\right)} \leqslant \widetilde{C} .
$$

This will be established (independently) in (6.66) below.

2') Let us infer from (6.44) that, for all $\sigma \in[0, s]$,

$$
\|F(\psi) u\|_{\mathcal{K}_{\nu}^{\sigma}(T)} \leqslant \widetilde{C}\|u\|_{\mathcal{K}_{\nu}^{\sigma}(T)},
$$

To see this write

$$
\begin{aligned}
\|F(\psi) u\|_{\mathcal{K}_{\nu}^{\sigma}(T)} & :=\|F(\psi) u\|_{L^{\infty}\left(0, T ; H^{\sigma-1}\right)}+\nu\|F(\psi) u\|_{L^{2}\left(0, T ; H^{\sigma}\right)} \\
& \leqslant\|F(\psi) u\|_{L^{\infty}\left(0, T ; H^{\sigma-1}\right)}+\|F(\psi) u\|_{L^{2}\left(0, T ; H_{\nu}^{\sigma}\right)} \\
& \leqslant \widetilde{C}\left\{\|u\|_{L^{\infty}\left(0, T ; H^{\sigma-1}\right)}+\|u\|_{L^{2}\left(0, T ; H_{\nu}^{\sigma}\right)}\right\} \leqslant \widetilde{C}\|u\|_{\mathcal{K}_{\nu}^{\sigma}(T)} .
\end{aligned}
$$

3) Let $\sigma \in[0, s], f \in H^{\sigma-1}(\mathbb{D})$ and $\gamma: \mathbb{D} \rightarrow(0,+\infty)$ be a function bounded from below by a positive constant. Furthermore, suppose $\tilde{\gamma}:=\gamma-\underline{\gamma} \in H^{s}(\mathbb{D})$ for some constant $\underline{\gamma}$. We claim that if $u \in H^{\sigma}(\mathbb{D})$ satisfies $\operatorname{div}(\bar{\gamma} \nabla u)=f$, then there exists a constant $K=K(d, s)$ such that

$$
\|u\|_{H^{\sigma+1}} \leqslant K\left\|\gamma^{-1}\right\|_{L^{\infty}}\left\{\|f\|_{H^{\sigma-1}}+\|\tilde{\gamma}\|_{H^{s}}\|u\|_{L^{2}}\right\}+\|u\|_{L^{2}} .
$$

Commuting the equation $\operatorname{div}(\gamma \nabla u)=f$ with $\Lambda^{\sigma}$ and using the commutator estimate (3.10) to bound $\operatorname{div}\left(\left[\gamma, \Lambda^{\sigma}\right] \nabla u\right)$, we see that the proof of (6.47) can be reduced to the special case $\sigma=0$. On the other hand, the case $\sigma=0$ is immediate by usual integration by parts and duality arguments.

b) Hereafter, RHS denotes the right-hand side of (6.42). To prove that

$$
\|\operatorname{div} \widetilde{v}\|_{\mathcal{K}_{\nu}^{\sigma}(T)} \leqslant \mathrm{RHS}
$$

we begin by showing that

$$
\|\operatorname{div} \widetilde{v}\|_{\mathcal{K}_{\nu}^{\sigma}(T)} \leqslant \widetilde{C}\left\{\left\|\left(\varepsilon \partial_{t}\right) \widetilde{p}\right\|_{\mathcal{K}_{\nu}^{\sigma}(T)}+\|\kappa \widetilde{\theta}\|_{\mathcal{K}_{\nu}^{\sigma+2}(T)}+\varepsilon\left\|f_{1}\right\|_{\mathcal{K}_{\nu}^{\sigma}(T)}\right\} .
$$

Rewrite $\operatorname{div} \widetilde{v}$ as

$$
-g_{1}(\phi)\left(\varepsilon \partial_{t}\right) \widetilde{p}+\kappa \mathcal{E}_{1}(\phi) \Delta \widetilde{\theta}+\kappa \mathcal{E}_{2}(\phi, \nabla \phi) \nabla \widetilde{\theta}+\varepsilon f_{1},
$$


where $\mathcal{E}_{1}$ and $\mathcal{E}_{2}$ are as in (6.31). Using (6.46), one has

$$
\begin{aligned}
\left\|g_{1}(\phi)\left(\varepsilon \partial_{t}\right) \widetilde{p}\right\|_{\mathcal{K}_{\nu}^{\sigma}(T)} & \leqslant \widetilde{C}\left\|\left(\varepsilon \partial_{t}\right) \widetilde{p}\right\|_{\mathcal{K}_{\nu}^{\sigma}(T)}, \\
\left\|\kappa \mathcal{E}_{1}(\phi) \Delta \widetilde{\theta}\right\|_{\mathcal{K}_{\nu}^{\sigma}(T)} & \leqslant \widetilde{C}\|\kappa \widetilde{\theta}\|_{\mathcal{K}_{\nu}^{\sigma+2}(T)} .
\end{aligned}
$$

Therefore, to infer (6.49) it remains only to estimate the $\mathcal{K}_{\nu}^{\sigma}(T)$-norm of $\kappa \mathcal{E}_{2}(\phi, \nabla \phi) \nabla \tilde{\theta}$. Using the very definition of $\mathcal{E}_{2}(\phi, \nabla \phi)$ and the estimate (6.45), one has

$$
\left\|\mathcal{E}_{2}(\phi, \nabla \phi)\right\|_{L^{\infty}\left(0, T ; H^{s-1}\right)} \lesssim \widetilde{C} .
$$

Since $s-1>d / 2$, the estimate (6.43) applies with $\sigma_{0}:=s-1$. It yields (6.50)

$$
\left\|\mathcal{E}_{2}(\phi, \nabla \phi) \nabla \widetilde{\theta}\right\|_{L^{\infty}\left(0, T ; H^{\sigma-1}\right)} \leqslant \widetilde{C}\|\nabla \widetilde{\theta}\|_{L^{\infty}\left(0, T ; H^{\sigma-1}\right)} \leqslant \widetilde{C}\|\widetilde{\theta}\|_{L^{\infty}\left(0, T ; H^{\sigma}\right)} .
$$

Moreover, one can easily verify that

$$
\begin{aligned}
& \nu\left\|\mathcal{E}_{2}(\phi, \nabla \phi) \nabla \widetilde{\theta}\right\|_{L^{2}\left(0, T ; H^{\sigma}\right)} \\
& \quad \leqslant\left\|\nu \mathcal{E}_{2}(\phi, \nabla \phi)\right\|_{L^{2}\left(0, T ; H^{s}\right)}\|\nabla \widetilde{\theta}\|_{L^{\infty}\left(0, T ; H^{\sigma}\right)} \\
& \quad \leqslant \sqrt{T}\left\|\mathcal{E}_{2}(\phi, \nabla \phi)\right\|_{L^{\infty}\left(0, T ; H_{\nu}^{s}\right)}\|\widetilde{\theta}\|_{L^{\infty}\left(0, T ; H^{\sigma+1}\right)} \\
& \leqslant \sqrt{T} C\left(\|\phi\|_{L^{\infty}\left(0, T ; H_{\nu}^{s+1}\right)}\right)\|\widetilde{\theta}\|_{L^{\infty}\left(0, T ; H^{\sigma+1}\right)} \\
& \leqslant \sqrt{T} C(\Omega)\|\widetilde{\theta}\|_{L^{\infty}\left(0, T ; H^{\sigma+1}\right)} \leqslant \widetilde{C}\|\widetilde{\theta}\|_{L^{\infty}\left(0, T ; H^{\sigma+1}\right)} .
\end{aligned}
$$

From this together with (6.50), we conclude that

$$
\left\|\mathcal{E}_{2}(\phi, \nabla \phi) \nabla \widetilde{\theta}\right\|_{\mathcal{K}_{\nu}^{\sigma}(T)} \leqslant \widetilde{C}\|\widetilde{\theta}\|_{L^{\infty}\left(0, T ; H^{\sigma+1}\right)} .
$$

This completes the proof of $\left(\underline{6.49)}\right.$ since $\|\widetilde{\theta}\|_{L^{\infty}\left(0, T ; H^{\sigma+1}\right)} \leqslant\|\widetilde{\theta}\|_{\mathcal{K}_{\nu}^{\sigma+2}(T)}$. So to prove (6.48) it remains only to show that

$$
\|\kappa \widetilde{\theta}\|_{\mathcal{K}_{\nu}^{\sigma}(T)} \leqslant \text { RHS. }
$$

To see this, solve the first equation in (6.41) for $\operatorname{div} v$ and substitute the result in the third equation, to obtain

$$
g_{3}(\phi) \partial_{t} \widetilde{\theta}-\kappa\left(B_{3}(\phi)-B_{1}(\phi)\right) \widetilde{\theta}=f_{3}+f_{3}^{\prime},
$$

with $f_{3}^{\prime}:=g_{1}(\phi)\left(\varepsilon \partial_{t}\right) \widetilde{p}-\varepsilon f_{1}$. Since

$$
B_{3}(\phi)-B_{1}(\phi):=\left(\chi_{3}(\varepsilon p)-\chi_{1}(\varepsilon p)\right) \operatorname{div}(\beta(\theta) \nabla \cdot),
$$

the assumption $\chi_{1}(\wp)<\chi_{3}(\wp)$ (which is (A3) in Assumption 2.1) implies that the equation (6.52) is parabolic. Hence, one can use the following estimate (see (A.2) below):

$$
\begin{aligned}
\|\kappa \widetilde{\theta}\|_{\mathcal{K}_{\nu}^{\sigma+2}(T)} & \leqslant\|\sqrt{\kappa} \nu \widetilde{\theta}\|_{L^{\infty}\left(0, T ; H^{\sigma+1}\right)}+\sqrt{\kappa}\|\sqrt{\kappa} \nu \widetilde{\theta}\|_{L^{2}\left(0, T ; H^{\sigma+2}\right)} \\
& \leqslant \widetilde{C}\left\{\|\sqrt{\kappa} \nu \tilde{\theta}(0)\|_{H^{\sigma+1}}+\nu\left\|f_{3}+f_{3}^{\prime}\right\|_{L^{2}\left(0, T ; H^{\sigma}\right)}\right\} .
\end{aligned}
$$

From which we easily infer the desired bound (6.51). 
c) Estimate for $\nabla \widetilde{p}$. Note that, by combining (6.46) with (6.47), one has

$$
\|\nabla \widetilde{p}\|_{\mathcal{K}_{\nu}^{\sigma}(T)} \leqslant \widetilde{C}\left\|\operatorname{div}\left(g_{2}(\phi)^{-1} \nabla \widetilde{p}\right)\right\|_{\mathcal{K}_{\nu}^{\sigma-1}(T)}+\widetilde{C}\|\widetilde{p}\|_{L^{\infty}\left(0, T ; L^{2}\right)} .
$$

Starting from $\nabla \widetilde{p}=-g_{2}(\phi)\left(\varepsilon \partial_{t}\right) \widetilde{v}+\varepsilon \mu B_{2}(\phi) \widetilde{v}+\varepsilon f_{2}$, it is found that

$$
\begin{aligned}
& \operatorname{div}\left(g_{2}(\phi)^{-1} \nabla \widetilde{p}\right)=-\left(\varepsilon \partial_{t}\right) \operatorname{div} \widetilde{v}+\varepsilon \mu \mathcal{F}_{1}(\phi) \nabla^{2} \operatorname{div} \widetilde{v} \\
& \quad+\varepsilon \mu \mathcal{F}_{2}(\phi, \nabla \phi) \nabla^{2} \widetilde{v}+\varepsilon \mu \mathcal{F}_{3}\left(\phi, \nabla \phi, \nabla^{2} \phi\right) \nabla \widetilde{v}+\varepsilon \operatorname{div}\left(g_{2}(\phi)^{-1} f_{2}\right),
\end{aligned}
$$

for some $C^{\infty}$ functions $\mathcal{F}_{1}, \mathcal{F}_{2}$ and $\mathcal{F}_{3}$ vanishing at the origin.

The most direct estimates show that the $\mathcal{K}_{\nu}^{\sigma-1}(T)$-norms of the first and the last three terms in the right-hand side of $[6.55$ are bounded by RHS. So we need only concentrate on the second term. Since $0 \leqslant \sigma-1 \leqslant s$, the estimate (6.46) implies

$$
\left\|\varepsilon \mu \mathcal{F}_{1}(\phi) \nabla^{2} \operatorname{div} \widetilde{v}\right\|_{\mathcal{K}_{\nu}^{\sigma-1}(T)} \leqslant \widetilde{C}\left\|\varepsilon \mu \nabla^{2} \operatorname{div} \widetilde{v}\right\|_{\mathcal{K}_{\nu}^{\sigma-1}(T)} .
$$

The following observation is important since this where the spectral localization of $\widetilde{U}$ enters. Since $\widetilde{v}=J_{\varepsilon \nu / 3} \widetilde{v}$ and since $\varepsilon \mu \nabla^{2} J_{\varepsilon \nu / 3} \lesssim \Lambda^{1}$, one has $\left\|\varepsilon \mu \nabla^{2} \operatorname{div} \widetilde{v}\right\|_{\mathcal{K}_{\nu}^{\sigma-1}(T)} \lesssim\|\operatorname{div} \widetilde{v}\|_{\mathcal{K}_{\nu}^{\sigma}(T)}$. The piece of information already determined in (6.48) implies that this in turn is $\leqslant$ RHS. Hence, the right-hand side of (6.54) is $\leqslant$ RHS. The proof of (6.42) is complete.

Recall that the upshot of the two previous lemma is to estimate the fast components $\operatorname{div} J_{\varepsilon \nu} v$ and $\nabla J_{\varepsilon v} p$.

Lemma 6.16. There exists a generic function $C(\cdot)$ such that

$$
\begin{aligned}
\left\|J_{\varepsilon \nu} p\right\|_{L^{\infty}\left(0, T ; H^{s}\right)}+\nu\left\|J_{\varepsilon \nu} p\right\|_{L^{2}\left(0, T ; H^{s+1}\right)} & \leqslant \widetilde{C} \\
\left\|\operatorname{div} J_{\varepsilon \nu} v\right\|_{L^{\infty}\left(0, T ; H^{s-1}\right)}+\nu\left\|\operatorname{div} J_{\varepsilon \nu} v\right\|_{L^{2}\left(0, T ; H^{s}\right)} & \leqslant \widetilde{C} .
\end{aligned}
$$

Proof. To avoid repetitions, we just give the scheme of the analysis. Set

$$
X_{m}:=\left\|p_{m}\right\|_{\mathcal{K}_{\nu}^{s-m+1}(T)}+\left\|\operatorname{div} v_{m}\right\|_{\mathcal{K}_{\nu}^{s-m}(T)} .
$$

Setting $\widetilde{U}:=U_{m}$ and $f:=f_{\mathrm{LF}}^{m}-G(\phi) v \cdot \nabla U_{m}$, where $G(\phi)$ is as defined in (5.23), we are in the situation of the previous lemma and hence the estimate (6.42) easily implies $X_{m} \leqslant \widetilde{C} X_{m+1}+Y_{m}$ where

$$
\begin{aligned}
Y_{m} \leqslant & \widetilde{C}\left\{\left\|U_{m}\right\|_{\mathcal{H}_{a}^{0}(T)}+\left\|U_{m}(0)\right\|_{\mathcal{H}_{a, 0}^{s-m}}\right\} \\
& +(\varepsilon+\sqrt{T}) C(\Omega)\left\{\left\|f_{\mathrm{LF}}^{m}\right\|_{L^{\infty}\left(0, T ; \mathcal{H}_{a, 0}^{s-1-m}\right)}+\left\|U_{m}\right\|_{\mathcal{H}_{a}^{s-m}(T)}\right\} .
\end{aligned}
$$

Gathering the results of the previous lemma, one obtains

$$
Y_{m} \leqslant \widetilde{C}\left\{\widetilde{C}+C\left(\Omega_{0}\right)\right\}+(\varepsilon+\sqrt{T}) C(\Omega)\{C(\Omega)+C(\Omega)\} \leqslant \widetilde{C} .
$$

Hence, we end up with $X_{m} \leqslant \widetilde{C} X_{m+1}+\widetilde{C}$. By an elementary induction, we therefore obtain $X_{0} \leqslant \widetilde{C} X_{S}+\widetilde{C}$. Finally, noting that $X_{S} \lesssim\left\|U_{s}\right\|_{\mathcal{H}_{a}^{0}(T)}$ and using Lemma 6.12 leads to the desired bound $X_{0} \leqslant \widetilde{C}$. 
6.5. The slow components. Having proved the estimates for the fast components, we now prove the estimates for the slow components $\theta$ and $\operatorname{curl} v$. To prove both estimates we use Assumption 2.2. This furnishes us with a $C^{\infty}$ function $S=S(\vartheta, \wp)$ such that $S(0,0)=0$ and

$$
\begin{aligned}
& \mathrm{d} S(\vartheta, \wp)=g_{3}(\vartheta, \wp) \mathrm{d} \vartheta-g_{1}(\vartheta, \wp) \mathrm{d} \wp, \\
& (\vartheta, \wp) \mapsto(S(\vartheta, \wp), \wp) \text { is a } C^{\infty} \text { change of variables. }
\end{aligned}
$$

[Recall that $(\vartheta, \wp) \in \mathbb{R}^{2}$ is the place holder of $(\theta, \varepsilon p)$.]

Notation 6.17. Set $\sigma:=S(\theta, \varepsilon p)$.

One reason it is interesting to introduce the coordinate $S=S(\vartheta, \wp)$ is that $\sigma$ is well transported by the flow (see also Remarks 6.20] and 6.22).

Lemma 6.18. Given $F \in C^{\infty}(\mathbb{R})$ satisfying $F(0)=0$, there exists a generic function $C(\cdot)$ such that

$$
\begin{array}{r}
\left\|\left(\partial_{t}+v \cdot \nabla\right) F(\sigma)\right\|_{L^{2}\left(0, T ; H^{s}\right)} \leqslant C(\Omega), \\
\|F(\sigma)\|_{L^{\infty}\left(0, T ; H^{s}\right)} \leqslant \widetilde{C} .
\end{array}
$$

Proof. Firstly, we form an evolution equation for $\sigma$. Directly from the identity (6.58), one has $\partial_{t, x} \sigma=g_{3}(\phi) \partial_{t, x} \theta-\varepsilon g_{1}(\phi) \partial_{t, x} p$. By combining the first and the last equations in (2.1) with this identity, we compute

$$
\partial_{t} \sigma+v \cdot \nabla \sigma-\kappa\left(\chi_{3}(\varepsilon p)-\chi_{1}(\varepsilon p)\right) \operatorname{div}(\beta(\theta) \nabla \theta)=0 .
$$

Therefore, one has

$$
\partial_{t} \sigma+v \cdot \nabla \sigma=\kappa G_{1}(\phi, \nabla \theta)+\kappa G_{2}(\phi) \Delta \theta,
$$

for some $C^{\infty}$ functions $G_{1}$ and $G_{2}$, with $G_{1}(0)=0$. Hence, the Moser estimate (5.8) implies at once

$$
\left\|\partial_{t} \sigma+v \cdot \nabla \sigma\right\|_{H^{s}} \leqslant C\left(\|\phi\|_{H^{s}}\right)\left\{1+\kappa\|\nabla \theta\|_{H^{s+1}}\right\} .
$$

Since $\sqrt{\kappa} \leqslant \nu$, one has

$$
\kappa\|\nabla \theta\|_{H^{s+2}} \leqslant \sqrt{\kappa}\|\nabla \theta\|_{H_{\nu}^{s+1}}
$$

so that $\left\|\partial_{t} \sigma+v \cdot \nabla \sigma\right\|_{H^{s}} \leqslant C(R)\left\{1+R^{\prime}\right\}$ where $R$ and $R^{\prime}$ are as defined in (6.20). Since $\Omega \approx\|R\|_{L^{\infty}(0, T)}+\left\|R^{\prime}\right\|_{L^{2}(0, T)}$, we have

$$
\left\|\left(\partial_{t}+v \cdot \nabla\right) \sigma\right\|_{L^{2}\left(0, T ; H^{s}\right)} \leqslant C(\Omega) .
$$

The chain rule and the rule of product in Sobolev spaces imply that the left-hand side of (6.60) is estimated by

$$
K\left(1+\left\|\widetilde{F^{\prime}}(\sigma)\right\|_{L^{\infty}\left(0, T ; H^{s}\right)}\right)\left\|\left(\partial_{t}+v \cdot \nabla\right) \sigma\right\|_{L^{2}\left(0, T ; H^{s}\right)} .
$$

where $\widetilde{F^{\prime}}(z)=F^{\prime}(z)-F^{\prime}(0)$ where $F^{\prime}$ denotes the differential of $F$. The first term in (6.64) is bounded by $C\left(\|\sigma\|_{L^{\infty}\left(0, T ; H^{s}\right)}\right) \leqslant C(\Omega)$ (here is where we use the hypothesis that $S(0,0)=0)$. Hence, (6.60) follows from (6.63). 
Moving to the proof of (6.61), we first recall an usual estimate in Sobolev spaces for hyperbolic equations ${ }^{5}$ : For all functions $u, \chi \in C^{0}\left([0, T] ; H^{s}(\mathbb{D})\right)$, with $s>1+d / 2$, there holds

$$
\|u\|_{L^{\infty}\left(0, T ; H^{s}\right)} \lesssim e^{T X}\|u(0)\|_{H^{s}}+\int_{0}^{T} e^{(T-t) X}\left\|\partial_{t} u+\chi \cdot \nabla u\right\|_{H^{s}} d t
$$

where $X:=K \int_{0}^{T}\|\chi\|_{H^{s}} d t$ for some constant $K$ depending only on $(s, d)$. The Cauchy-Schwarz inequality readily implies that the right-hand side is estimated by

$$
e^{K T\|\chi\|_{L^{\infty}\left(0, T ; H^{s}\right)}}\left\{\|u(0)\|_{H^{s}}+\sqrt{T}\left\|\partial_{t} u+\chi \cdot \nabla u\right\|_{L^{2}\left(0, T ; H^{s}\right)}\right\} .
$$

Applying this bound with $(u, \chi)=(F(\sigma), v)$, it is found that the $L^{\infty}\left(0, T ; H^{s}\right)$ norm of $F(\sigma)$ is estimated by

$$
e^{K T \Omega}\left\{C\left(\Omega_{0}\right)+\sqrt{T}\left\|\partial_{t} F(\sigma)+v \cdot \nabla F(\sigma)\right\|_{L^{2}\left(0, T ; H^{s}\right)}\right\} .
$$

We complete the proof of (6.61) by using (6.60).

Corollary 6.19. Given $F \in C^{\infty}\left(\mathbb{R}^{2+d}\right)$ satisfying $F(0)=0$, there exists a generic function $C(\cdot)$ such that

$$
\|F(\psi)\|_{L^{\infty}\left(0, T ; H^{s}\right)} \leqslant \widetilde{C} .
$$

Proof. The property (6.59) implies that there exists a $C^{\infty}$ function $F^{*}$ such that $F(\vartheta, \wp, \mathrm{v})=F^{*}(S(\vartheta, \wp), \wp, \mathrm{v})$ for all $(\vartheta, \wp, \mathrm{v}) \in \mathbb{R} \times \mathbb{R} \times \mathbb{R}^{d}$. Moreover, the hypothesis $S(0,0)=0$ implies $F^{*}(0,0,0)=0$.

Decompose $F(\psi):=F(\theta, \varepsilon p, \varepsilon v)=F^{*}(\sigma, \varepsilon p, \varepsilon v)$ as

$$
F^{*}(\sigma, 0,0)+\left\{F^{*}(\sigma, \varepsilon p, \varepsilon v)-F^{*}(\sigma, 0,0)\right\} .
$$

The first term is estimated by way of (6.61). To bound the second term, observe that one can factor out $(\varepsilon p, \varepsilon v)$. Consequently, there exists a function $C(\cdot)$ depending only on $F^{*}$ such that

$$
\left\|F^{*}(\sigma, \varepsilon p, \varepsilon v)-F^{*}(\sigma, 0)\right\|_{H^{s}} \leqslant \varepsilon C\left(\|(\theta, p, v)\|_{H^{s}}\right) \leqslant \varepsilon C(R) \leqslant e^{\varepsilon C\left(\|R\|_{L \infty(0, T)}\right)} .
$$

Which completes the proof.

Remark 6.20. The estimate (5.21) implies $\left\|\partial_{t} F(\psi)\right\|_{L^{2}\left(0, T ; H_{\nu}^{s}\right)} \leqslant C(\Omega)$. As in (4.22), it yields $\|F(\psi)\|_{L^{\infty}\left(0, T ; H_{\nu}^{s}\right)} \leqslant C\left(\Omega_{0}\right)+\sqrt{T} C(\Omega) \leqslant \widetilde{C}$. Yet, this is weaker than (6.66) [indeed $H_{0}^{s}=H^{s-1}$ ].

Now we really use the special feature of the low frenquency analysis. The following lemma states that $\Lambda_{\varepsilon \nu}^{-1} \sigma$ satisfies parabolic-type estimates.

Lemma 6.21. There exists a generic function $C(\cdot)$ such that

$$
\left\|\Lambda_{\varepsilon \nu}^{-1} \sigma\right\|_{L^{\infty}\left(0, T ; H_{\nu}^{s+1}\right)}+\sqrt{\kappa}\left\|\Lambda_{\varepsilon \nu}^{-1} \sigma\right\|_{L^{2}\left(0, T ; H_{\nu}^{s+2}\right)} \leqslant \widetilde{C} .
$$

\footnotetext{
${ }^{5}$ See also the estimate A.2 with $\eta=0$.
} 
Proof. In light of the estimate $\|\sigma\|_{L^{\infty}\left(0, T ; H^{s}\right)} \leqslant \widetilde{C}$ (see (6.61)), it suffices to prove that $\dot{\sigma}:=\nu \Lambda_{\varepsilon \nu}^{-1} \nabla \sigma$ satisfies

$$
\|\dot{\sigma}\|_{L^{\infty}\left(0, T ; H^{s}\right)}+\sqrt{\kappa}\|\dot{\sigma}\|_{L^{2}\left(0, T ; H^{s+1}\right)} \leqslant \widetilde{C} .
$$

Let us form a parabolic evolution equation for $\dot{\sigma}:=\nu \Lambda_{\varepsilon \nu}^{-1} \nabla \sigma$. Writing the identity (6.58) in the form $\mathrm{d} \vartheta=c_{1}(\vartheta, \wp) \mathrm{d} S+c_{2}(\vartheta, \wp) \mathrm{d} \wp$ with $c_{1}:=1 / g_{3}^{-1}$ and $c_{2}:=g_{1} / g_{3}$, yields

$$
\nabla \theta=c_{1}(\phi) \nabla \sigma+\varepsilon c_{2}(\phi) \nabla p .
$$

Inserting this expression for $\nabla \theta$ into the equation (6.62), yields

$$
\partial_{t} \sigma+v \cdot \nabla \sigma-\kappa k(\phi) \Delta \sigma=\kappa \mathrm{G}_{3}+\kappa \mathrm{G}_{4},
$$

where $k:=\left(\chi_{3}-\chi_{1}\right) \beta c_{1}$ is a smooth positive function ${ }^{6}$ and

$$
\mathrm{G}_{3}+\mathrm{G}_{4}:=G_{3}(\phi, \nabla \phi)+\varepsilon G_{4}(\phi, \nabla \phi) \Delta p,
$$

for some $C^{\infty}$ functions $G_{3}$ and $G_{4}$, with $G_{3}(0)=0$.

We compute

$$
\partial_{t} \dot{\sigma}+v \cdot \nabla \dot{\sigma}-\kappa k(\phi) \Delta \dot{\sigma}=\mathcal{G},
$$

where the source term is given by

$$
\begin{aligned}
\mathcal{G}:= & -\nu \Lambda_{\varepsilon \nu}^{-1}(\nabla v \cdot \nabla \sigma)+\nu\left[v, \Lambda_{\varepsilon \nu}^{-1}\right] \cdot \nabla \nabla \sigma \\
& +\kappa \Lambda_{\varepsilon \nu}^{-1}(\nabla k(\phi) \Delta \sigma)+\kappa \nu\left[k(\phi), \Lambda_{\varepsilon \nu}^{-1}\right] \Delta \nabla \sigma \\
& +\nu \kappa \Lambda_{\varepsilon \nu}^{-1} \nabla \mathrm{G}_{3}+\nu \kappa \Lambda_{\varepsilon \nu}^{-1} \nabla \mathrm{G}_{4} .
\end{aligned}
$$

Let us give the scheme of the analysis. In light of the standard estimate (A.2) (given in the appendix below), to prove (6.67) we need only show that the source term $\mathcal{G}$ can be split as $\mathcal{G}_{1}+\sqrt{\kappa} \mathcal{G}_{2}$ with

$$
\left\|\mathcal{G}_{1}\right\|_{L^{1}\left(0, T ; H^{s}\right)} \leqslant \widetilde{C} \text { and }\left\|\mathcal{G}_{2}\right\|_{L^{2}\left(0, T ; H^{s-1}\right)} \leqslant \widetilde{C} .
$$

To see this, there are only two nontrivial points. Firstly, note that

$$
\begin{aligned}
& \left\|\nu \sqrt{\kappa} \Lambda_{\varepsilon \nu}^{-1} \nabla \mathrm{G}_{4}\right\|_{L^{2}\left(0, T ; H^{s-1}\right)} \\
& :=\left\|\nu \sqrt{\kappa} \Lambda_{\varepsilon \nu}^{-1} \nabla\left\{\varepsilon G_{4}(\phi, \nabla \phi) \Delta p\right\}\right\|_{L^{2}\left(0, T ; H^{s-1}\right)} \\
& \left.\lesssim\left\|\sqrt{\kappa} G_{4}(\phi, \nabla \phi) \Delta p\right\|_{L^{2}\left(0, T ; H^{s-1}\right)} \quad \text { (since } \varepsilon \nu \nabla \Lambda_{\varepsilon \nu}^{-1} \lesssim I\right) \\
& \lesssim\left(1+\left\|\tilde{G}_{4}(\phi, \nabla \phi)\right\|_{L^{\infty}\left(0, T ; H^{s-1}\right)}\right)\|\sqrt{\kappa} p\|_{L^{2}\left(0, T ; H^{s+1}\right)} \quad \text { (straightforward) } \\
& \lesssim \widetilde{C} \widetilde{C} \text {. } \\
& \text { (by (6.66), 6.56) ) }
\end{aligned}
$$

Secondly, there is a little trick: set $I_{\mu, \kappa}:=\nu /(\sqrt{\mu}+\sqrt{\kappa}) \leqslant 1$ and decompose $\nu \nabla v \cdot \nabla \sigma$ into two parts:

$$
\nu \nabla v \cdot \nabla \sigma=I_{\mu, \kappa} \sqrt{\mu} \nabla v \cdot \nabla \sigma+I_{\mu, \kappa} \sqrt{\kappa} \nabla v \cdot \nabla \sigma .
$$

\footnotetext{
${ }^{6}$ By hypotheses, one has $\chi_{1}<\chi_{3}, 0<c_{1}:=1 / g_{3}$ and $0<\beta$ (see Assumption 2.1).
} 
Using the usual Moser estimates as well as the weighted versions (5.6)-(5.7), it is easily found that

$$
\begin{aligned}
\sqrt{\mu}\|\nabla v \cdot \nabla \sigma\|_{H^{s}} & \lesssim\|\nabla v\|_{H_{\sqrt{\mu}}^{s}}\|\nabla \sigma\|_{H_{\sqrt{\mu}}^{s}} \lesssim\left(R+R^{\prime}\right) C(R), \\
\|\nabla v \cdot \nabla \sigma\|_{H^{s-1}} & \lesssim\|v\|_{H^{s}}\|\sigma\|_{H^{s}} \lesssim R C(R),
\end{aligned}
$$

so that

$$
\sqrt{\mu}\|\nabla v \cdot \nabla \sigma\|_{L^{1}\left(0, T ; H^{s}\right)}+\|\nabla v \cdot \nabla \sigma\|_{L^{2}\left(0, T ; H^{s-1}\right)} \leqslant \sqrt{T} C(\Omega) \leqslant \widetilde{C} .
$$

With these estimates established, the proof easily follows.

Remark 6.22. One interesting feature of the equation (6.69) for $\sigma$ is that it is coupled to the momentum equation only through the convective term. Indeed, for the purpose of proving estimates independent of $\kappa$, we cannot see the term $\operatorname{div} v$ [in the equation for $\theta$ ] as a source term.

We denote by $\operatorname{curl} v$ the matrix with coefficients $(\operatorname{curl} v)_{i j}:=\partial_{j} v_{i}-\partial_{i} v_{j}$. The basic idea of the forthcoming computations is to apply the curl operator to the equation for $v$ so as to cancel the large term $\varepsilon^{-1} \nabla p$. Yet, this requires some preparation because the factor $g_{2}(\theta, \varepsilon p)$ multiplying the time derivative of $v$ admits large oscillations in $\mathcal{O}(1)$. To get around this, we follow the analysis of [31]. Namely, as in the proof of Corollary 6.19] we decompose $g_{2}(\theta, \varepsilon p)$ into two parts: the first which is well transported by the flow, the second which admits small oscillations of typical size $\mathcal{O}(\varepsilon)$.

In particular, we do not estimate $\operatorname{curl} v$ directly. Instead we estimate $\operatorname{curl}\left(\gamma_{0} v\right)$ where the coefficient $\gamma_{0}$ is defined as follows.

Notation 6.23. By (6.59), one can write $g_{2}(\vartheta, \wp)=\Gamma(S(\vartheta, \wp), \wp)$ for some smooth positive function $\Gamma$. Set $\Gamma_{0}(\vartheta, \wp):=\Gamma(S(\vartheta, \wp), 0)$ and

$$
\gamma_{0}:=\Gamma_{0}(\theta, \varepsilon p) \text {. }
$$

Lemma 6.24. There exists a generic function $C(\cdot)$ such that

$$
\left\|\operatorname{curl}\left(\gamma_{0} v\right)\right\|_{L^{\infty}\left(0, T ; H^{s-1}\right)}+\sqrt{\mu}\left\|\operatorname{curl}\left(\gamma_{0} v\right)\right\|_{L^{2}\left(0, T ; H^{s}\right)} \leqslant \widetilde{C} .
$$

Proof. We begin by computing the equation satisfied by $\omega:=\operatorname{curl}\left(\gamma_{0} v\right)$. It follows from elementary calculus that there exists a $C^{\infty}$ function $\Gamma_{1}$ such that for all $(\wp, \vartheta) \in \mathbb{R}^{2}$

$$
\Gamma_{0}(\vartheta, \wp) / g_{2}(\vartheta, \wp)=1+\wp \Gamma_{1}(\vartheta, \wp) .
$$

We first insert the expression for $g_{2}(\theta, \varepsilon p)$ given by (6.73) into the equation for $v$, thereby obtaining

$$
\gamma_{0}\left(\partial_{t} v+v \cdot \nabla v\right)+\varepsilon^{-1} \nabla p-\mu B_{2} v=F^{\dagger},
$$

with $F^{\dagger}:=-p \gamma_{1} \nabla p+\varepsilon \mu p \gamma_{1} B_{2} v$ where $\gamma_{1}:=\Gamma_{1}(\theta, \varepsilon p)$. Consequently, the equation for $v$ is equivalent to

$$
\left(\partial_{t}+v \cdot \nabla\right)\left(\gamma_{0} v\right)+\varepsilon^{-1} \nabla p-\underset{56}{\mu B_{2} v}=F^{\dagger}+\left(\partial_{t} \gamma_{0}+v \cdot \nabla \gamma_{0}\right) v
$$


Using the elementary identity $\operatorname{curl} \nabla=0$, we find

$$
\left(\partial_{t}+v \cdot \nabla\right) \operatorname{curl}\left(\gamma_{0} v\right)-\mu B_{2} \operatorname{curl} v=F^{\dagger \dagger},
$$

where

$F^{\dagger \dagger}:=\operatorname{curl} F^{\dagger}-[\operatorname{curl}, v] \cdot \nabla\left(\gamma_{0} v\right)+\mu\left[\operatorname{curl}, B_{2}\right] v+\operatorname{curl}\left(v\left(\partial_{t} \gamma_{0}+v \cdot \nabla \gamma_{0}\right)\right)$.

Next, write $B_{2} \operatorname{curl}=\gamma_{0}^{-1} \gamma_{0} B_{2} \operatorname{curl}=\gamma_{0}^{-1} B_{2} \operatorname{curl}\left(\gamma_{0} \cdot\right)+\gamma_{0}^{-1}\left[\gamma_{0}, B_{2} \operatorname{curl}\right]$ to obtain that $\omega=\operatorname{curl}\left(\gamma_{0} v\right)$ satisfies

$$
\partial_{t} \omega+v \cdot \nabla \omega-\mu \gamma_{0}^{-1} B_{2} \omega=F
$$

with $F:=F^{\dagger \dagger}+\mu \gamma_{0}^{-1}\left[\gamma_{0}, B_{2} \operatorname{curl}\right] v$.

To sum up, $F=\sum_{1 \leqslant i \leqslant} F_{i}$ with

$$
\begin{array}{ll}
F_{1}:=-\operatorname{curl}\left(p \gamma_{1} \nabla p\right), & F_{4}:=\mu\left[\operatorname{curl}, B_{2}\right] v, \\
F_{2}:=\varepsilon \mu \operatorname{curl}\left(p \gamma_{1} B_{2} v\right), & F_{5}:=-[\operatorname{curl}, v] \cdot \nabla\left(\gamma_{0} v\right), \\
F_{3}:=\operatorname{curl}\left(v\left(\partial_{t} \gamma_{0}+v \cdot \nabla \gamma_{0}\right)\right), & F_{6}:=\mu \gamma_{0}^{-1}\left[\gamma_{0}, B_{2} \operatorname{curl}\right] v .
\end{array}
$$

Estimate for $F$. As in the proof of Lemma 6.21 to prove (6.72) it suffices to show that one can decompose $F$ as $f_{1}+\sqrt{\mu} f_{2}$ with

$$
\left\|f_{1}\right\|_{L^{1}\left(0, T ; H^{s-1}\right)} \leqslant \widetilde{C} \text { and }\left\|f_{2}\right\|_{L^{2}\left(0, T ; H^{s-2}\right)} \leqslant \widetilde{C} .
$$

To do so we decompose $F$ as $f_{1}+\sqrt{\mu} f_{2}$ with

$$
\left\|f_{1}\right\|_{L^{2}\left(0, T ; H^{s-1}\right)} \leqslant C(\Omega) \text { and }\left\|f_{2}\right\|_{L^{\infty}\left(0, T ; H^{s-2}\right)} \leqslant C(\Omega) .
$$

[Note that (6.76) implies (6.75) since $\sqrt{T} C(\Omega) \leqslant \widetilde{C}$.]

Set $f_{2}:=\varepsilon \sqrt{\mu} p \gamma_{1} \operatorname{curl}\left(B_{2} v\right)-\sqrt{\mu} \gamma_{0}^{-1} v \wedge B_{2} \nabla \gamma_{0}$ and $f_{1}:=F-f_{2}$. It follows from the very definition of $\Omega$ that $f_{2}$ satisfies the second estimate in (6.76). As regards $f_{1}$, the key point is the following: Starting from

$$
\left\|F_{3}\right\|_{H^{s-1}} \lesssim\|v\|_{H^{s}}\left\|\partial_{t} \gamma_{0}+v \cdot \nabla \gamma_{0}\right\|_{H^{s}}
$$

we obtain $\left\|F_{3}\right\|_{L^{2}\left(0, T ; H^{s-1}\right)} \lesssim\|v\|_{L^{\infty}\left(0, T ; H^{s}\right)}\left\|\partial_{t} \gamma_{0}+v \cdot \nabla \gamma_{0}\right\|_{L^{2}\left(0, T ; H^{s}\right)}$. As a consequence, using the very definition of $\gamma_{0}$ (see (6.71) ), the estimate (6.60) implies that $\left\|F_{3}\right\|_{L^{2}\left(0, T ; H^{s-1}\right)} \leqslant C(\Omega)$.

Let us estimate the other terms without repeated uses of the rules of product. To do so set $P:=(p, \nabla p), V:=(v, \nabla v)$ and $\Theta:=(\theta, \nabla \theta)$. Direct computations show that one can write $F_{1}+F_{2}+F_{4}+F_{5}+F_{6}-f_{2}$ as

$$
\sum\left(\sqrt{\mu} \partial_{x}\right)^{\alpha} G_{\alpha, a}(P, V, \Theta) \quad\left(\alpha \in \mathbb{N}^{d},|\alpha| \leqslant 1\right),
$$

for some $C^{\infty}$ functions $G_{\alpha, a}$ such that the family $\left\{G_{\alpha, a}|a \in A,| \alpha \mid \leqslant 1\right\}$ is bounded in $C^{\infty}$, with in addition $G_{0, a}(0)=0$. The Moser estimate (5.9) implies

$$
\begin{aligned}
\left\|\left(\sqrt{\mu} \partial_{x}\right)^{\alpha} G_{\alpha, a}(P, V, \Theta)\right\|_{H^{s-1}} & \leqslant\left\|G_{\alpha, a}(P, V, \Theta)\right\|_{H_{\sqrt{\mu}}^{s}} \\
& \leqslant C\left(\|(P, V, \Theta)\|_{L^{\infty}}\right)\|(P, V, \Theta)\|_{H_{\sqrt{\mu}}^{s}} .
\end{aligned}
$$


Hence, directly from the definition of $\Omega$, we arise at the desired bound

$$
\left\|\left(\sqrt{\mu} \partial_{x}\right)^{\alpha} G_{\alpha, a}(P, V, \Theta)\right\|_{L^{2}\left(0, T ; H^{s-1}\right)} \leqslant C(\Omega) .
$$

Which completes the proof of the claim (6.76)

6.6. End of the proof of Proposition 6.1. In order to prove Proposition 6.1 it remains to estimate $J_{\varepsilon \nu} \theta$ and $J_{\varepsilon \nu} v$.

Lemma 6.25. Given $F \in C^{\infty}\left(\mathbb{R}^{2}\right)$ such that $F(0)=0$, there exists a generic function $C(\cdot)$ such that

$$
\left\|J_{\varepsilon \nu} F(\phi)\right\|_{L^{\infty}\left(0, T ; H_{\nu}^{s+1}\right)}+\sqrt{\kappa}\left\|J_{\varepsilon \nu} \nabla F(\phi)\right\|_{L^{2}\left(0, T ; H_{\nu}^{s+1}\right)} \leqslant \widetilde{C} .
$$

Remark 6.26. With $F(\phi)=\theta$ this is the expected bound for $J_{\varepsilon \nu} \theta$.

Proof. In light of (6.61), it suffices to prove

$$
\nu\left\|J_{\varepsilon \nu} \nabla F(\phi)\right\|_{L^{\infty}\left(0, T ; H^{s}\right)}+\nu \sqrt{\kappa}\left\|J_{\varepsilon \nu} \nabla^{2} F(\phi)\right\|_{L^{2}\left(0, T ; H^{s}\right)} \leqslant \widetilde{C} .
$$

By (6.59) one has

$$
\nabla F(\phi)=F_{1}(\phi) \nabla \sigma+\varepsilon F_{2}(\phi) \nabla p,
$$

for some $C^{\infty}$ functions $F_{1}$ and $F_{2}$. Applying Proposition 3.2 with $\sigma_{0}=s$, $\sigma_{1}=\sigma_{2}=m_{1}=0$ and $m_{2}=1$ leads to

$$
\begin{aligned}
\left\|\nu \nabla \Lambda_{\varepsilon \nu}^{-1} F(\phi)\right\|_{H^{s}} \lesssim & \left(1+\left\|\tilde{F}_{1}(\phi)\right\|_{H^{s}}\right)\left\|\nabla \nu \Lambda_{\varepsilon \nu}^{-1} \sigma\right\|_{H^{s}} \\
& +\left(1+\left\|\tilde{F}_{2}(\phi)\right\|_{H^{s}}\right)\left\|\nabla \varepsilon \nu \Lambda_{\varepsilon \nu}^{-1} p\right\|_{H^{s}},
\end{aligned}
$$

where $\tilde{F}:=F-F(0)$. Using $\nu\|\cdot\|_{H^{s}} \leqslant\|\cdot\|_{H_{\nu}^{s}}, \varepsilon \nu \nabla \Lambda_{\varepsilon \nu}^{-1} \lesssim I$ and (6.61), there results

$$
\left\|\nu \nabla \Lambda_{\varepsilon \nu}^{-1} F(\phi)\right\|_{L^{\infty}\left(0, T ; H^{s}\right)} \leqslant \widetilde{C}\left\{\left\|\Lambda_{\varepsilon \nu}^{-1} \sigma\right\|_{L^{\infty}\left(0, T ; H_{\nu}^{s+1}\right)}+\|p\|_{L^{\infty}\left(0, T ; H^{s}\right)}\right\} .
$$

As a consequence, from Lemma 6.21 and (6.56), we get the first half of (6.78), namely: $\left\|\nu J_{\varepsilon \nu} \nabla F(\phi)\right\|_{L^{\infty}\left(0, T ; H^{s}\right)} \leqslant \widetilde{C}$.

The technique for obtaining the second half is similar. Differentiate (6.79), to obtain: $\partial_{i} \partial_{j} F(\phi):=\mathcal{F}_{I}+\mathcal{F}_{I I}$ with

$$
\begin{aligned}
\mathcal{F}_{I} & :=F_{1}(\phi) \partial_{i} \partial_{j} \sigma+\varepsilon F_{2}(\phi) \partial_{i} \partial_{j} p, \\
\mathcal{F}_{I I} & :=\partial_{i} F_{1}(\phi) \partial_{j} \sigma+\varepsilon \partial_{i} F_{2}(\phi) \partial_{j} p .
\end{aligned}
$$

Exactly as above, one has

$$
\begin{aligned}
\nu \sqrt{\kappa}\left\|J_{\varepsilon \nu} \mathcal{F}_{I}\right\|_{L^{2}\left(0, T ; H^{s+2}\right)} & \lesssim \widetilde{C}\left\{\nu \sqrt{\kappa}\left\|\Lambda_{\varepsilon \nu}^{-1} \sigma\right\|_{L^{2}\left(0, T ; H^{s+2}\right)}+\sqrt{\kappa}\|p\|_{L^{2}\left(0, T ; H^{s+1}\right)}\right\} \\
& \leqslant \widetilde{C} \widetilde{C} .
\end{aligned}
$$


In order to estimate $\mathcal{F}_{I I}$, note that $\mathcal{F}_{I I}:=G(\phi, \nabla \phi)$ for some $C^{\infty}$ function $G$ such that $G(0)=0$. Hence, one has

$$
\begin{aligned}
\nu \sqrt{\kappa}\left\|\mathcal{F}_{I I}\right\|_{L^{2}\left(0, T ; H^{s}\right)} & \lesssim \sqrt{T}\|G(\phi, \nabla \phi)\|_{L^{\infty}\left(0, T, H_{\nu}^{s}\right)} & & \text { (straightforward) } \\
& \lesssim \sqrt{T} C\left(\|\phi\|_{L^{\infty}\left(0, T ; H_{\nu}^{s+1}\right)}\right) & & \text { (by (15.7)) } \\
& \lesssim \sqrt{T} C(\Omega) \leqslant \widetilde{C} & & \text { (by definition). }
\end{aligned}
$$

This completes the proof.

The next estimate finishes the proof of Proposition 6.1

Lemma 6.27. There exists a generic function $C(\cdot)$ such that

$$
\left\|J_{\varepsilon \nu} v\right\|_{L^{\infty}\left(0, T ; H_{\varepsilon \nu}^{s+1}\right)}+\sqrt{\mu}\left\|J_{\varepsilon \nu} \nabla v\right\|_{L^{2}\left(0, T ; H_{\varepsilon \nu}^{s+1}\right)} \leqslant \widetilde{C} .
$$

Proof. We need only explain how to combine all the previous Lemma.

a) To shorten notations, given a function $f$ such that $f-\underline{f} \in H^{\sigma}$ for some constant $\underline{f}$, we denote by $\|f\|_{\dot{H}^{\sigma}}$ the norm $\|f-\underline{f}\|_{H^{\sigma}}$. The semi-norm $\|\cdot\|_{\dot{H}_{\nu}^{\sigma}}$ is defined in the same way.

We claim that for all smooth positive function $\gamma$, there exists a constant $K$ such that for all $(\varepsilon, \nu)$ and for all vector field $u \in H^{S}(\mathbb{D})$, one has

$$
\begin{aligned}
\left\|J_{\varepsilon \nu} u\right\|_{H^{s}} \leqslant K M^{2 s+1}\{ & \left\|\operatorname{div} J_{\varepsilon \nu} u\right\|_{H^{s-1}}+\|\operatorname{curl}(\gamma u)\|_{H^{s-1}} \\
& \left.+\left\|J_{\varepsilon \nu} u\right\|_{L^{2}}+\varepsilon\|u\|_{H^{s}}\right\},
\end{aligned}
$$

where $M:=1+\left\|\gamma^{-1}\right\|_{\dot{H}^{s}}+\|\gamma\|_{\dot{H}_{\nu}^{s+1}}$.

To prove this result, we start from the following estimate: for all $\sigma \geqslant 0$,

$$
\|u\|_{H^{\sigma+1}} \lesssim\|\operatorname{div} u\|_{H^{\sigma}}+\|\operatorname{curl} u\|_{H^{\sigma}}+\|u\|_{L^{2}} .
$$

As curl $u=\gamma^{-1} \operatorname{curl}(\gamma u)-\gamma^{-1} \nabla \gamma \wedge u$, by the usual rule of product in Sobolev spaces, we infer that for all $\sigma \in[0, s-1]$,

$$
\begin{aligned}
\|\operatorname{curl} u\|_{H^{\sigma}} & \lesssim\left(1+\left\|\gamma^{-1}\right\|_{\dot{H}^{s}}\right)\left\{\|\operatorname{curl}(\gamma u)\|_{H^{\sigma}}+\|\gamma\|_{\dot{H}^{s}}\|u\|_{H^{\sigma}}\right\} \\
& \lesssim M\|\operatorname{curl}(\gamma u)\|_{H^{\sigma}}+M^{2}\|u\|_{H^{\sigma}} .
\end{aligned}
$$

Thus, the estimate (6.81) turns into

$$
\|u\|_{H^{\sigma+1}} \lesssim M^{2}\left\{\|\operatorname{div} u\|_{H^{\sigma}}+\|\operatorname{curl}(\gamma u)\|_{H^{\sigma}}+\|u\|_{H^{\sigma}}\right\} .
$$

By induction on $\mathbb{N} \ni \sigma \leqslant s-1$, it yields

$$
\|u\|_{H^{s}} \lesssim M^{2 s}\left\{\|\operatorname{div} u\|_{H^{s-1}}+\|\operatorname{curl}(\gamma u)\|_{H^{s-1}}+\|u\|_{L^{2}}\right\} .
$$

Furthermore, by (3.11) applied with $\left(m, \sigma_{0}, \sigma\right)=(1, s+1, s)$, one has

$$
\begin{aligned}
\left\|\operatorname{curl}\left(\gamma J_{\varepsilon \nu} u\right)\right\|_{H^{s-1}} & \leqslant\left\|J_{\varepsilon \nu} \operatorname{curl}(\gamma u)\right\|_{H^{s-1}}+\left\|\left[\gamma, J_{\varepsilon \nu}\right] u\right\|_{H^{s}} \\
& \lesssim\|\operatorname{curl}(\gamma u)\|_{H^{\sigma}}+\varepsilon \nu\|\gamma\|_{\dot{H}^{s+1}}\|u\|_{H^{s}} .
\end{aligned}
$$

From the estimate $\nu\|\gamma\|_{\dot{H}^{s+1}} \leqslant\|\gamma\|_{\dot{H}_{\nu}^{s+1}} \leqslant M$ and (6.82) applied with $u$ replaced by $J_{\varepsilon \nu} u$, the previous fact implies (6.80). 
b) Let $\gamma:=\gamma_{0}$ (as defined in (6.71) $)$ and let $M:=1+\left\|\gamma^{-1}\right\|_{\dot{H}^{s}}+\|\gamma\|_{\dot{H}_{\nu}^{s+1}}$. We list our bounds:

by Lemma 6.25:

$\|M\|_{L^{\infty}(0, T)} \leqslant \widetilde{C}$,

by 6.57) :

by Lemma 6.24:

$\left\|\operatorname{div} J_{\varepsilon \nu} v\right\|_{L^{\infty}\left(0, T ; H^{s-1}\right)} \leqslant \widetilde{C}$,

by Lemma 6.12:

$\left\|\operatorname{curl}\left(\gamma_{0} v\right)\right\|_{L^{\infty}\left(0, T ; H^{s-1}\right)} \leqslant \widetilde{C}$

directly from the definitions : $\quad\|\varepsilon v\|_{L^{\infty}\left(0, T ; H^{s}\right)} \leqslant \varepsilon \Omega \leqslant \widetilde{C}$.

Hence, we deduce from (6.80) that $\left\|J_{\varepsilon \nu} v\right\|_{L^{\infty}\left(0, T ; H^{s}\right)} \leqslant \widetilde{C}$. This in turn implies $\left\|J_{\varepsilon \nu} v\right\|_{L^{\infty}\left(0, T ; H_{\varepsilon \nu}^{s+1}\right)} \leqslant \widetilde{C}$, indeed recall that $\left\|J_{\varepsilon \nu} v\right\|_{H_{\varepsilon \nu}^{\sigma+1}} \lesssim\left\|J_{\varepsilon \nu} v\right\|_{H^{\sigma}}$. c) By (6.81), (6.57) and Lemma 6.12, to prove $\sqrt{\mu}\left\|J_{\varepsilon \nu} \nabla v\right\|_{L^{2}\left(0, T ; H_{\varepsilon \nu}^{s+1}\right)} \leqslant \widetilde{C}$ we need only check that $\sqrt{\mu}\left\|\operatorname{curl} J_{\varepsilon \nu} v\right\|_{L^{2}\left(0, T ; H^{s}\right)} \leqslant \widetilde{C}$. For this purpose, write

$$
\left\|\operatorname{curl} J_{\varepsilon \nu} v\right\|_{H^{s}} \lesssim\left(1+\left\|\gamma_{0}^{-1}\right\|_{\dot{H}^{s}}\right)\left\{\left\|\operatorname{curl}\left(\gamma_{0} J_{\varepsilon \nu} v\right)\right\|_{H^{s}}+\left\|\gamma_{0}\right\|_{\dot{H}^{s+1}}\|v\|_{H^{s}}\right\},
$$

and

$$
\left\|\operatorname{curl}\left(\gamma_{0} J_{\varepsilon \nu} v\right)\right\|_{H^{s}} \lesssim\left\|\operatorname{curl}\left(\gamma_{0} v\right)\right\|_{H^{s}}+\left\|\gamma_{0}\right\|_{\dot{H}^{s+1}}\|v\|_{H^{s}},
$$

where we used the commutator estimate (3.11) with $\left(m, \sigma_{0}, \sigma\right)=(0, s+1, s)$. These two facts and the estimate $\sqrt{\mu}\left\|\gamma_{0}\right\|_{L^{\infty}\left(0, T ; \dot{H}^{s+1}\right)} \leqslant\left\|\gamma_{0}\right\|_{L^{\infty}\left(0, T ; \dot{H}_{\nu}^{s+1}\right)} \leqslant$ $\widetilde{C}$ (see Lemma 6.25) implies

$$
\sqrt{\mu}\left\|\operatorname{curl} J_{\varepsilon \nu} v\right\|_{L^{2}\left(0, T ; H^{s}\right)} \leqslant \widetilde{C}\left\{\sqrt{\mu}\left\|\operatorname{curl}\left(\gamma_{0} v\right)\right\|_{L^{2}\left(0, T ; H^{s}\right)}+\|v\|_{L^{2}\left(0, T ; H^{s}\right)}\right\} .
$$

Hence, the expected bound directly follows from Lemma 6.24 and the estimate $\|v\|_{L^{2}\left(0, T ; H^{s}\right)} \leqslant \sqrt{T}\|v\|_{L^{\infty}\left(0, T ; H^{s}\right)} \leqslant \widetilde{C}$.

\section{UNIFORM STABILITY}

In this section we prove Theorem 2.7. Granted the uniform a priori bounds proved in the previous sections, the proof essentially reduces to establishing a local existence result for fixed $a \in A$.

7.1. Local existence result. For fixed $a \in A$, the fact that the Cauchy problem for (1.1) is well-posed is immediate provided that one chooses to work with the unknown $(\rho, v, \mathcal{T})$. Indeed, with this choice of dependent variables the system is a coupled hyperbolic/parabolic system in symmetric form, so that the general theory applies. We begin by proving that this remains valid for the system (2.1).

Introduce first a definition clarifying the structure of the systems we produce. Consider a system of nonlinear equations

$$
A_{0}(u) \partial_{t} u+\sum_{\alpha \in \mathbb{N}^{d}, 1 \leqslant|\alpha| \leqslant 2} A_{\alpha}(u) \partial_{x}^{\alpha} u=F(u, \nabla u),
$$


where each $A_{\beta}(0 \leqslant|\beta| \leqslant 2)$ is a $n \times n$ matrix smooth in its arguments and furthermore symmetric: $A_{\beta}=A_{\beta}^{t}$. We suppose $F$ is smooth in its arguments, with values in $\mathbb{R}^{n}$.

Definition 7.1. The system (7.1) is said to be of coupled hyperbolic/parabolic type provided that there exists $\left(n_{1}, n_{2}\right) \in \mathbb{N}^{2}$, and a splitting of the unknowns $u=\left(u^{1}, u^{2}\right) \in \mathbb{R}^{n_{1}} \times \mathbb{R}^{n_{2}}$ so that $F(u, \nabla u)=\left(0, F^{2}(u, \nabla u)\right)^{t}$ and

$$
A_{0}(u)=\left(\begin{array}{cc}
A_{0}^{11}(u) & 0 \\
0 & A_{0}^{22}(u)
\end{array}\right), \quad A_{\alpha}(u)=\left(\begin{array}{cc}
0 & 0 \\
0 & A_{\alpha}^{22}(u)
\end{array}\right) \quad \text { for all }|\alpha|=2,
$$

where in addition

$$
\forall(u, \xi) \in \mathbb{R}^{n} \times \mathbb{S}^{d-1}, \quad A_{0}^{11}(u)>0, \quad A_{0}^{22}(u)>0, \quad-\sum_{|\alpha|=2} A_{\alpha}^{22}(u) \xi^{\alpha}>0 .
$$

(here $M^{j k}$ denotes the sub-blocks of the matrix $M$ which corresponds to the splitting $\left.\mathbb{R}^{n} \ni u=\left(u^{1}, u^{2}\right) \in \mathbb{R}^{n_{1}} \times \mathbb{R}^{n_{2}}\right)$.

The general theory 28, 40] for hyperbolic or parabolic problems applies for coupled hyperbolic/parabolic ones. One can prove the following result.

Proposition 7.2. P1) Let $s>2+d / 2$. Assume that (7.1) is of coupled hyperbolic/parabolic type. Then for all initial data $u_{0}$ in $H^{S}(\mathbb{D})$ there exists a positive $T=T\left(\left\|u_{0}\right\|_{H^{s}}\right)$ such that the Cauchy problem for (7.1) has a unique classical solution $\Phi\left(u_{0}\right) \in C^{0}\left([0, T] ; H^{S}(\mathbb{D})\right)$ such that $\Phi^{2}\left(u_{0}\right) \in$ $L^{2}\left(0, T ; H^{s+1}(\mathbb{D})\right)$ where $\Phi^{2}\left(u_{0}\right)$ are the last $n_{2}$ components of $\Phi\left(u_{0}\right)$ where $n_{2}$ is as in Definition 7.1.

P2) The interval $\left[0, T^{\star}\right)$, with $T^{\star}<+\infty$, is a maximal interval of $H^{s}$ existence if and only if $\lim \sup _{t \rightarrow T^{\star}}\left\|\Phi\left(u_{0}\right)(t)\right\|_{W^{1, \infty}(\mathbb{D})}=+\infty$.

Remark 7.3. i. If the system is symmetric hyperbolic, it is enough to assume $s>1+d / 2$.

ii. Let $u \in C^{0}\left([0, T] ; H^{S}(\mathbb{D})\right)$ be a classical solution of (7.1). It follows from property P2) that, if $u(0) \in H^{\infty}(\mathbb{D})$ then $u \in C^{\infty}\left([0, T] ; H^{\infty}(\mathbb{D})\right)$.

Fix $a=(\varepsilon, \mu, \kappa) \in A$. We next show that an appropriate change of variables transforms the system (2.1) into a system of coupled hyperbolic/parabolic type. Let $\varrho$ denotes the function given by Assumption 2.2 Since the mapping $\mathbb{R}^{2} \ni(\vartheta, \wp) \mapsto(\vartheta, \varrho(\vartheta, \wp)) \in \mathbb{R}^{2}$ is a $C^{\infty}$ diffeomorphism, it is equivalent to work with $(p, v, \theta)$ or with $(\rho, v, \theta)$ where $\rho:=\varrho(\theta, \varepsilon p)$.

Firstly, we form an evolution equation for $\rho:=\varrho(\theta, \varepsilon p)$. By combining the first and the last equations in (2.1) with the second identity in (2.2) written in the form $\chi_{3} \mathrm{~d} \varrho=\chi_{3} g_{1} \mathrm{~d} \wp-\chi_{1} g_{3} \mathrm{~d} \vartheta$, we get

$$
\chi_{3}\left(\partial_{t} \rho+v \cdot \nabla \rho\right)+\left(\chi_{3}-\chi_{1}\right) \operatorname{div} v=0 .
$$

The second identity in (2.2) written in the form

$$
\mathrm{d} \wp=\gamma_{1} \mathrm{~d} \vartheta+\gamma_{2} \mathrm{~d} \varrho:=\frac{\chi_{1} g_{3}}{\chi_{3} g_{1}} \mathrm{~d} \vartheta+\frac{1}{g_{1}} \mathrm{~d} \varrho,
$$


yields $\varepsilon \nabla p=\gamma_{1} \nabla \theta+\gamma_{2} \nabla \rho$. Summing up, one has

$$
\left\{\begin{array}{l}
\chi_{3}\left(\partial_{t} \rho+v \cdot \nabla \rho\right)+\left(\chi_{3}-\chi_{1}\right) \operatorname{div} v=0, \\
g_{2}\left(\partial_{t} v+v \cdot \nabla v\right)+\varepsilon^{-2} \gamma_{1} \nabla \theta+\varepsilon^{-2} \gamma_{2} \nabla \rho-\mu B_{2} v=0, \\
g_{3}\left(\partial_{t} \theta+v \cdot \nabla \theta\right)+\operatorname{div} v-\kappa B_{3} \theta=0
\end{array}\right.
$$

where all the coefficients are positive by Assumption 2.1. We also mention that all the coefficients are evaluated at $(\theta, \varepsilon p)=(\theta, \mathrm{P}(\theta, \rho))$ for some $C^{\infty}$ function $P$. We next symmetrize the system (7.3). To do so multiply the first equation by $\left(\chi_{3}-\chi_{1}\right)^{-1} \gamma_{1}$, the second by $\varepsilon^{2}$ and the third one by $\gamma_{2}$, to obtain

$$
\left\{\begin{array}{l}
\delta_{1}\left(\partial_{t} \rho+v \cdot \nabla \rho\right)+\gamma_{1} \operatorname{div} v=0 \\
\delta_{2}\left(\partial_{t} v+v \cdot \nabla v\right)+\gamma_{1} \nabla \rho+\gamma_{2} \nabla \theta-\varepsilon^{2} \mu B_{2} v=0 \\
\delta_{3}\left(\partial_{t} \theta+v \cdot \nabla \theta\right)+\gamma_{2} \operatorname{div} v-\kappa \gamma_{2} B_{3} \theta=0
\end{array}\right.
$$

with $\delta_{1}:=\left(\chi_{3}-\chi_{1}\right)^{-1} \gamma_{1} \chi_{3}, \delta_{2}:=\varepsilon^{2} g_{2}$ and $\delta_{3}:=\gamma_{2} g_{3}$ [compare with the system [4.15]. Again, by Assumption 2.1] the coefficients $\delta_{i}$ 's are positive.

Lemma 7.4. For all $(\mu, \kappa) \in[0,1]^{2}$, the symmetric system (7.4) is of coupled hyperbolic/parabolic type.

Proof. Set $n=2+d$. If $\mu=\kappa=0$ set $n_{1}=n$ and $u^{1}=u$. If $\mu=0$ and $\kappa>0$ set $n_{1}=1+d$ and $u^{1}=(\rho, v)$. If $\mu>0$ and $\kappa=0$ set $n_{1}=2$ and $u^{1}=(\rho, \theta)$. If $\mu>0$ and $\kappa>0$ set $n_{1}=1$ and $u^{1}=\rho$.

With this result in hands, we obtain that the Cauchy problem for (17.4) is well-posed for all fixed $a \in A$, so is the Cauchy problem for (2.1) since (7.4) has been deduced from (2.1) by using a $C^{\infty}$ diffeomorphism.

We are now prepared to prove Theorem 2.7

7.2. Proof of Theorem 2.7. Let $s>1+d / 2$ and $M_{0}>0$. The previous analysis implies that, for all $a \in A$, there exists $T_{a}=T_{a}\left(M_{0}\right)$ such that for all initial data $U_{0}$ in the ball $B\left(\mathcal{H}_{a, 0}^{s}, M_{0}\right)$, the Cauchy problem for (2.1) has a unique classical solution $U_{a}$ in $\mathcal{H}_{a}^{s}\left(T_{a}\right)$. Denotes by $T_{a}^{\star}$ the maximal time of existence of such a classical solution. For all $t<T_{a}^{\star}$, set $\Omega_{a}(t):=$ $\left\|U_{a}\right\|_{\mathcal{H}_{a}^{\mathrm{s}}(t)}$. Our task is to show that there exists $T>0$ and $M<+\infty$ such that

$$
\forall a \in A, \quad T_{a}^{\star} \geqslant T \text { and } \Omega_{a}(T) \leqslant M .
$$

Up to replacing the initial data $U_{0}$ by $J_{\delta} U_{0}$ and letting $\delta$ goes to zero, we can assume that $U_{0} \in H^{\infty}(\mathbb{D})$. In light of Remark 7.3, it means that we can assume $U_{a} \in C^{\infty}\left(\left[0, T_{a}^{\star}\right) ; H^{\infty}(\mathbb{D})\right)$. By combining Propositions 5.1 and 6.1. we know that there exists a smooth non-decreasing function $C(\cdot)$ such that for all $a \in A$ and all $t<\min \left\{T_{a}^{\star}, 1\right\}$, there holds

$$
\Omega_{a}(t) \leqslant C\left(\Omega_{a}(0)\right) e^{(\sqrt{t}+\varepsilon) C\left(\Omega_{a}(t)\right)} .
$$


Choose first $M_{1}>C\left(M_{0}\right)$ and next $T_{1} \leqslant 1$ and $\varepsilon_{1} \leqslant 1$ such that

$$
C\left(M_{0}\right) e^{\left(\sqrt{T_{1}}+\varepsilon_{1}\right) C\left(M_{1}\right)}<M_{1} \text {. }
$$

Let $t<\min \left\{T_{a}^{\star}, T_{1}\right\}$ and $a=(\varepsilon, \mu, \kappa) \in A$ be such that $\varepsilon \leqslant \varepsilon_{1}$. By combining the inequalities (7.6) and (7.7) with the hypothesis $\Omega_{a}(0)<M_{0}$, we infer that $\Omega_{a}(t) \neq M_{1}$. Besides, we can assume without restriction that $M_{0}<M_{1}$, so that $\Omega_{a}(0)<M_{1}$. Since the function $\Omega_{a}$ is continuous, we infer $\Omega_{a}(t)<M_{1}$. Consequently, the continuation principle [which is P2) in Proposition 7.2 shows that $T_{a}^{\star}>T_{1}$.

On the other hand the [omitted] proof of Proposition 7.2 implies that there exists $T_{2}>0$ and $M_{2}<+\infty$ such that for all $a=(\varepsilon, \mu, \kappa) \in A$ with $\varepsilon \geqslant \varepsilon_{1}$, one has $T_{a}^{\star} \geqslant T_{2}$ and $\Omega_{a}\left(T_{2}\right) \leqslant M_{2}$.

We have proved (7.5) with $T:=\min \left\{T_{1} / 2, T_{2}\right\}$ and $M:=\max \left\{M_{1}, M_{2}\right\}$, which completes the proof of Theorem [2.7]

\section{Decay of the LOCAL EnERGy}

We will consider systems which include (2.1) as a special case. The motivation is to consider a structure general enough to include, say, the combustion equations. To do so we allow the limit constraint on the divergence of the velocity field to read $\operatorname{div} v=F\left(D^{m} \psi\right)$ where $\psi$ is the slow variable (namely $\left.\partial_{t} \psi=\mathcal{O}(1)\right), m \geqslant 0$ is a given integer and

$$
D^{m} \psi:=\left\{\partial_{x}^{\alpha} \psi\left|\alpha \in \mathbb{N}^{d},\right| \alpha \mid \leqslant m\right\} .
$$

More precisely, we consider system of the form:

$$
\left\{\begin{array}{l}
g_{1}(\psi) \partial_{t} p+\frac{1}{\varepsilon} \operatorname{div} v=\frac{1}{\varepsilon} F_{1}\left(D^{m} \psi\right)+F_{2}\left(D^{m} \psi, D^{m} p, D^{m} v\right), \\
g_{2}(\psi) \partial_{t} v+\frac{1}{\varepsilon} \nabla p=F_{3}\left(D^{m} \psi, D^{m} p, D^{m} v\right), \\
\partial_{t} \psi=F_{4}\left(D^{m} \psi, D^{m} p, D^{m} v\right),
\end{array}\right.
$$

where $(p, v, \psi)$ is defined on $[0, T] \times \mathbb{R}^{d}$ with values in $\mathbb{R} \times \mathbb{R}^{d} \times \mathbb{R}^{n}, n \geqslant 0$ and $m \geqslant 0$ are given integers.

Assumption 8.1. The functions $g_{i}(i=1,2)$ and $F_{j}(j=1,2,3,4)$ are smooth in their arguments, satisfying

$$
g_{1}(y), g_{2}(y)>0, \quad F_{1}(Y), F_{2}(Y, Z) \in \mathbb{R}, \quad F_{3}(Y, Z) \in \mathbb{R}^{d}, \quad F_{4}(Y, Z) \in \mathbb{R}^{n},
$$

where $y, Y$ and $Z$ are the place holders of $\psi, D^{m} \psi$ and $\left(D^{m} p, D^{m} v\right)$. Moreover $F_{j}(0)=0$ for $j=1,2,3,4$.

Assuming that the slow variable decays sufficiently rapidly at spatial infinity we prove that the penalized terms converge to 0 .

Proposition 8.2. Let $T>0, m \geqslant 0$ and $s>2 m+d / 2$. Assume that the functions $\left(p^{\varepsilon}, v^{\varepsilon}, \psi^{\varepsilon}\right)$ satisfy (8.1) and

$$
\left\|\left(p^{\varepsilon}, v^{\varepsilon}, \psi^{\varepsilon}\right)\right\|_{\mathcal{K}^{s}(T)}:=\sup _{t \in[0, T]}\left\|\left(p^{\varepsilon}, v^{\varepsilon}\right)(t)\right\|_{H^{s}}+\left\|\psi^{\varepsilon}(t)\right\|_{H^{s+m-1}}<+\infty .
$$


Assume further that $\psi^{\varepsilon}$ converges strongly in $C^{0}\left([0, T] ; H_{\text {loc }}^{\sigma}\left(\mathbb{R}^{d}\right)\right)$, for some $\sigma>1+d / 2$, to a limit $\psi$ satisfying, for all $(t, x) \in[0, T] \times \mathbb{R}^{d}$,

$$
|\psi(t, x)| \leqslant K|x|^{-1-\gamma}, \quad|\nabla \psi(t, x)| \leqslant K|x|^{-2-\gamma},
$$

for some positive constants $K$ and $\gamma$. Then, $p^{\varepsilon}$ converges strongly to 0 in $L^{2}\left(0, T ; H_{l o c}^{s^{\prime}}\left(\mathbb{R}^{d}\right)\right)$ for all $s^{\prime}<s$ and $\operatorname{div} v^{\varepsilon}-F_{1}\left(D^{m} \psi^{\varepsilon}\right)$ converges strongly to 0 in $L^{2}\left(0, T ; H_{l o c}^{s^{\prime}}\left(\mathbb{R}^{d}\right)\right)$ for all $s^{\prime}<s-1$.

Proof. Since $\left(p^{\varepsilon}, v^{\varepsilon}, \psi^{\varepsilon}\right)$ is uniformly bounded in $\mathcal{K}^{\mathrm{S}}(T)$ (see (8.2)), using an interpolation argument, it is sufficient to prove that $p^{\varepsilon}$ and $\operatorname{div} v^{\varepsilon}-$ $F_{1}\left(D^{m} \psi^{\varepsilon}\right)$ converges strongly to 0 in $L^{2}\left(0, T ; L_{l o c}^{2}\left(\mathbb{R}^{d}\right)\right)$. To prove this result we use the following Theorem proved in 31] [although not explicitly stated in this way]. To clarify matters, we mention that: (1) all the functions considered in the following statement are real-valued; (2) all the convergences considered in this proof are strong.

Theorem 8.3 (Métivier \& Schochet). Let $T>0$ and let $u^{\varepsilon}$ be a bounded sequence in $C^{0}\left([0, T] ; H^{2}\left(\mathbb{R}^{d}\right)\right)$ such that

$$
\varepsilon^{2} \partial_{t}\left(a^{\varepsilon} \partial_{t} u^{\varepsilon}\right)-\operatorname{div}\left(b^{\varepsilon} \nabla u^{\varepsilon}\right)=c^{\varepsilon},
$$

where the source term $c^{\varepsilon}$ converges to 0 in $L^{2}\left(0, T ; L^{2}\left(\mathbb{R}^{d}\right)\right)$. Assume further that, for some $\sigma>1+d / 2$, the coefficients $\left(a^{\varepsilon}, b^{\varepsilon}\right)$ are uniformly bounded in $C^{0}\left([0, T] ; H^{\sigma}\left(\mathbb{R}^{d}\right)\right)$ and converges in $C^{0}\left([0, T] ; H_{l o c}^{\sigma}\left(\mathbb{R}^{d}\right)\right)$ to a limit $(a, b)$ satisfying the decay estimate

$$
\begin{array}{ll}
|a(t, x)-\underline{a}| \leqslant K|x|^{-1-\gamma}, & |\nabla a(t, x)| \leqslant K|x|^{-2-\gamma}, \\
|b(t, x)-\underline{b}| \leqslant K|x|^{-1-\gamma}, & |\nabla b(t, x)| \leqslant K|x|^{-2-\gamma},
\end{array}
$$

for some given positive constants $\underline{a}, \underline{b}, K$ and $\gamma$.

Then, the sequence $u^{\varepsilon}$ converges to 0 in $L^{2}\left(0, T ; L_{l o c}^{2}\left(\mathbb{R}^{d}\right)\right)$.

We can directly apply Theorem 8.3 to prove the first half of Proposition 8.2. that is the convergence of $p^{\varepsilon}$ to 0 in $L^{2}\left(0, T ; L_{l o c}^{2}\left(\mathbb{R}^{d}\right)\right)$. Indeed, applying $\varepsilon^{2} \partial_{t}$ to the first equation in (8.1), we compute

$$
\varepsilon^{2} \partial_{t}\left(a^{\varepsilon} \partial_{t} p^{\varepsilon}\right)-\operatorname{div}\left(b^{\varepsilon} \nabla p^{\varepsilon}\right)=c^{\varepsilon}:=\varepsilon f^{\varepsilon},
$$

with $a^{\varepsilon}:=g_{1}\left(\psi^{\varepsilon}\right), b^{\varepsilon}:=1 / g_{2}\left(\psi^{\varepsilon}\right)$ and

$$
f^{\varepsilon}:=\partial_{t} F_{1}\left(Y^{\varepsilon}\right)+\varepsilon \partial_{t} F_{2}\left(Y^{\varepsilon}, Z^{\varepsilon}\right)-\operatorname{div}\left(b^{\varepsilon} F_{3}\left(Y^{\varepsilon}, Z^{\varepsilon}\right)\right),
$$

where $Y^{\varepsilon}:=D^{m} \psi^{\varepsilon}$ and $Z^{\varepsilon}:=\left(D^{m} p^{\varepsilon}, D^{m} v^{\varepsilon}\right)$.

The equations (8.1) imply that one can rewrite $f^{\varepsilon}$ as

$$
f^{\varepsilon}=\mathcal{F}\left(D^{m+1} p^{\varepsilon}, D^{m+1} v^{\varepsilon}, D^{m+1} \psi^{\varepsilon}\right),
$$

where $\mathcal{F}$ is a $C^{\infty}$ function such that $\mathcal{F}(0)=0$. Using the usual nonlinear estimate in Sobolev spaces (5.8), the hypothesis (8.2) implies that $f^{\varepsilon}$ is uniformly bounded in $C^{0}\left([0, T] ; H^{s-m-1}\left(\mathbb{R}^{d}\right)\right)$. Consequently, $c^{\varepsilon}=\varepsilon f^{\varepsilon}$ converges to 0 in $L^{2}\left(0, T ; L^{2}\left(\mathbb{R}^{d}\right)\right)$ and Theorem 8.3 applies. 
To prove the second half of Proposition 8.2 we begin by proving that $\widetilde{p}^{\varepsilon}:=\left(\varepsilon \partial_{t}\right) p^{\varepsilon}$ converges to 0 in $L^{2}\left(0, T ; L_{l o c}^{2}\left(\mathbb{R}^{d}\right)\right)$. To do so apply $\left(\varepsilon \partial_{t}\right)$ on the equation (8.4), to obtain

$$
\varepsilon^{2} \partial_{t}\left(a^{\varepsilon} \partial_{t} \widetilde{p}^{\varepsilon}\right)-\operatorname{div}\left(b^{\varepsilon} \nabla \widetilde{p}^{\varepsilon}\right)=\widetilde{c}^{\varepsilon}:=\varepsilon \widetilde{f}^{\varepsilon},
$$

with

$$
\tilde{f}^{\varepsilon}:=\varepsilon \partial_{t} f^{\varepsilon}-\varepsilon \partial_{t}\left(\partial_{t} a^{\varepsilon}\left(\varepsilon \partial_{t}\right) p^{\varepsilon}\right)+\operatorname{div}\left(\partial_{t} b^{\varepsilon} \nabla p^{\varepsilon}\right),
$$

where $f^{\varepsilon}$ is given by (8.5). Again one can verify that $\widetilde{f}^{\varepsilon}$ is a bounded sequence in $C^{0}\left([0, T] ; L^{2}\left(\mathbb{R}^{d}\right)\right)$, which proves the desired result.

To complete the proof, observe that

$$
\operatorname{div} v^{\varepsilon}-F_{1}\left(Y^{\varepsilon}\right)=-g_{1}\left(\psi^{\varepsilon}\right)\left(\varepsilon \partial_{t}\right) p^{\varepsilon}+\varepsilon F_{2}\left(Z^{\varepsilon}\right) .
$$

Hence, the fact that $\operatorname{div} v^{\varepsilon}-F_{1}\left(Y^{\varepsilon}\right)$ converges to 0 in $L^{2}\left(0, T ; L_{l o c}^{2}\left(\mathbb{R}^{d}\right)\right)$ follows from the previous step and the fact that $g_{1}\left(\psi^{\varepsilon}\right)-g_{1}(0)$ and $F_{2}\left(Z^{\varepsilon}\right)$ are uniformly bounded in $C^{0}\left([0, T] ; H^{s}\left(\mathbb{R}^{d}\right)\right)$.

8.1. Proof of Theorem 2.9. To simplify the presentation we concentrate on the hardest case when $\kappa$ is a fixed positive constant.

We first prove the convergences for some sub-sequence of $\left(p^{\varepsilon}, v^{\varepsilon}, \theta^{\varepsilon}\right)$.

The equation for $\theta$ implies that $\partial_{t} \theta$ is bounded in $C^{0}\left([0, T] ; H^{s-1}\left(\mathbb{R}^{d}\right)\right)$. Therefore after extracting a sub-sequence, we can assume that, for all $s^{\prime}<s$,

$$
\theta^{\varepsilon} \rightarrow \theta \quad \text { in } C^{0}\left([0, T] ; H_{l o c}^{s^{\prime}+1}\left(\mathbb{R}^{d}\right)\right),
$$

where the limit $\theta$ belongs to $C^{0}\left([0, T] ; H_{l o c}^{s^{\prime}+1}\left(\mathbb{R}^{d}\right)\right) \cap L^{\infty}\left(0, T ; H^{s+1}\left(\mathbb{R}^{d}\right)\right)$.

Since $\left(p^{\varepsilon}, v^{\varepsilon}\right)$ is uniformly bounded in $C^{0}\left([0, T] ; H^{S}\left(\mathbb{R}^{d}\right)\right)$, after extracting further sub-sequence, we can also assume that

$$
\left(p^{\varepsilon}, v^{\varepsilon}\right) \rightarrow(p, v) \quad \text { weakly } \star \text { in } L^{\infty}\left(0, T ; H^{s}\left(\mathbb{R}^{d}\right)\right) .
$$

Note that the system (8.1) includes system (2.1) as a special case where $\psi^{\varepsilon}:=\left(\theta^{\varepsilon}, \varepsilon p^{\varepsilon}\right)$ and $m=2$. It follows from the very definition of $\psi^{\varepsilon}$ and the norms $\|\cdot\|_{H_{\varrho}^{\sigma}}$ that

$$
\begin{aligned}
\left\|\left(p^{\varepsilon}, v^{\varepsilon}\right)\right\|_{H^{s}}+\left\|\psi^{\varepsilon}\right\|_{H^{s+m-1}} & \leqslant\left\|\left(p^{\varepsilon}, v^{\varepsilon}\right)\right\|_{H^{s}}+\varepsilon\left\|p^{\varepsilon}\right\|_{H^{s+1}}+\left\|\theta^{\varepsilon}\right\|_{H^{s+1}} \\
& \leqslant\left\|\left(p^{\varepsilon}, v^{\varepsilon}\right)\right\|_{H_{\varepsilon}^{s+1}}+\left\|\theta^{\varepsilon}\right\|_{H^{s+1}} \\
& \lesssim\left\|\left(p^{\varepsilon}, v^{\varepsilon}\right)\right\|_{H_{\varepsilon \nu}^{s+1}}+\left\|\theta^{\varepsilon}\right\|_{H_{\nu}^{s+1}}
\end{aligned}
$$

where the implicit constant depends only on the fixed positive value of $\nu:=$ $\sqrt{\mu+\kappa}$. It follows that $\left\|\left(p^{\varepsilon}, v^{\varepsilon}, \psi^{\varepsilon}\right)\right\|_{\mathcal{K}^{s}(T)} \lesssim\left\|\left(p^{\varepsilon}, v^{\varepsilon}, \theta^{\varepsilon}\right)\right\|_{\mathcal{H}_{(\varepsilon, \mu, \kappa)}^{s}(T)}$. As a consequence the first hypothesis of Proposition 8.2 is satisfied. Therefore, in order to apply Proposition 8.2 to the equations (2.1) so as to obtain Theorem 2.9. it only remains to show that $\psi=(\theta, 0)$ satisfies the crucial assumption (8.3). 
Multiplying the first equation in (2.1) by $\varepsilon$ and passing to the weak limit (in the sense of distributions) shows that $\operatorname{div} v^{\varepsilon}-\chi_{1}\left(\varepsilon p^{\varepsilon}\right) \operatorname{div}\left(\beta\left(\theta^{\varepsilon}\right) \nabla \theta^{\varepsilon}\right)$ converges to 0 in that sense. Therefore, the limit $\theta$ satisfies

$$
g_{3}(\theta, 0)\left(\partial_{t} \theta+v \cdot \nabla \theta\right)-\kappa\left(\chi_{3}(0)-\chi_{1}(0)\right) \operatorname{div}(\beta(\theta) \nabla \theta)=0 .
$$

The assumption $\chi_{3}>\chi_{1}$ [see (A3) in Assumption 2.1] implies that (8.8]) is parabolic. The desired spatial decay thus follows from estimates in weighted Sobolev spaces. Indeed, introduce $\theta_{\delta}:=\langle x\rangle^{\delta} \theta$ for some given $\delta>2$. Then

$$
\partial_{t} \theta_{\delta}-\operatorname{div}\left(k(\theta) \nabla \theta_{\delta}\right)=\sum V_{\alpha}(t, x) \partial_{x}^{\alpha} \theta_{\delta} \quad\left(\alpha \in \mathbb{N}^{d},|\alpha| \leqslant 1\right),
$$

for some smooth positive function $k$ and some coefficients $V_{\alpha}$ such that $\left\|V_{\alpha}\right\|_{H^{s}} \leqslant C\left(\|(v, \theta)\|_{H^{s}}\right)$. Commuting $\Lambda^{s}$ with the equation and applying the usual $L^{2}$ estimate, we obtain

$$
\left\|\theta_{\delta}\right\|_{L^{\infty}\left(0, T ; H^{s}\right)} \leqslant C e^{T C}\left\|\theta_{\delta}(0)\right\|_{H^{s}},
$$

for some constant $C$ depending only on $\|(v, \theta)\|_{L^{\infty}\left(0, T ; H^{s}\right)}$ (see (A.2) with $\eta=1)$. The convergence (8.6) implies that $\theta(0)$ is the limit $\theta_{0}$ of the initial data $\theta^{\varepsilon}(0)$. Using the hypothesis $\langle x\rangle^{\delta} \theta_{0} \in H^{s}\left(\mathbb{R}^{d}\right)$, it follows from (8.9) that

$$
\left\|\theta_{\delta}\right\|_{L^{\infty}\left(0, T ; H^{s}\right)}<+\infty .
$$

Since $s>1+d / 2$, the Sobolev Theorem implies that $H^{s}\left(\mathbb{R}^{d}\right) \hookrightarrow W^{1, \infty}\left(\mathbb{R}^{d}\right)$. Hence, the assumption (8.3) is satisfied.

A moment's thought shows that the convergences holds for the full sequence. The proof of Theorem 2.9 is complete.

\section{Appendix A. Estimates FOR HYPERBolic AND PARABOliC SYSTEMS}

Here we briefly recall some standard estimates for hyperbolic or parabolic systems we used throughout the paper. We state estimates for the solutions $u=u(t, x) \in \mathbb{R}^{n}$ of systems having the form:

$$
\partial_{t} u+\sum_{|\alpha|=1} A_{\alpha} \partial_{x}^{\alpha} u-\eta \sum_{|\alpha|=2} A_{\alpha} \partial_{x}^{\alpha} u=f_{1}+\sqrt{\eta} f_{2},
$$

where each $A_{\alpha}=A_{\alpha}(t, x)$ is a $n \times n$ matrix valued-function smooth in its arguments and furthermore symmetric: $A_{\beta}=A_{\beta}^{t}$; also, the source terms $f_{i}=f_{i}(t, x)$ are smooth functions with values in $\mathbb{R}^{n}$. In particular, when $\eta=0$, the system A.1 is symmetric hyperbolic. We assume that for $\eta>0$ the system is parabolic: for all triple $(t, x, \xi)$ with $\xi \neq 0$,

$$
\sum_{|\alpha|=2} A_{\alpha}(t, x) \xi^{\alpha}>0
$$


Lemma A.1. Let $0 \leqslant \sigma \leqslant s \in(1+d / 2,+\infty)$. There exists a function $C(\cdot)$ such that for all $T \in[0,1]$, all $u \in C^{1}\left([0, T] ; H^{\infty}(\mathbb{D})\right)$ satisfying (A.1) and all $\eta \in[0,1]$,

$$
\begin{aligned}
& \|u\|_{L^{\infty}\left(0, T ; H^{\sigma}\right)}+\sqrt{\eta}\|u\|_{L^{2}\left(0, T ; H^{\sigma+1}\right)} \\
& \quad \leqslant K e^{C(R) T}\left(\|u(0)\|_{H^{\sigma}}+\left\|f_{1}\right\|_{L^{1}\left(0, T: H^{\sigma}\right)}+\left\|f_{2}\right\|_{L^{2}\left(0, T ; H^{\sigma-1}\right)}\right),
\end{aligned}
$$

where $K=K(s, d)$ and $R:=\sum_{|\alpha| \leqslant 2}\left\|\tilde{A}_{\alpha}\right\|_{L^{\infty}\left(0, T ; H^{s}\right)}$ where $\tilde{A}=A-A(0)$.

Proof. Commuting the equation (A.1) with $\Lambda^{\sigma}$, using the commutator estimate (3.10) and the Gronwall's lemma, the proof of (A.2) can be reduced to the special case $\sigma=0$. The latter case relies upon usual integrations by parts and duality arguments.

Acknowledgments. I would like to express my sincere gratitude to G. Métivier for introducing me to the analysis of the low Mach number limit as well as for helpful discussions. I warmly thanks D. Bresch and D. Lannes for many advices. I am most appreciative for the hospitality shown to me by the Rennes university during the beginning of this work.

\section{REFERENCES}

[1] T. Alazard - "Incompressible limit of the nonisentropic Euler equations with solid wall boundary conditions", Adv. in Differential Equations 10 (2005), p. 19-44.

[2] J.-M. Bony - "Calcul symbolique et propagation des singularités pour les équations aux dérivées partielles non linéaires", Ann. Sci. École Norm. Sup. (4) 14 (1981), no. 2, p. 209-246.

[3] D. Bresch, B. Desjardins, E. Grenier \& C.-K. Lin - "Low Mach number limit of viscous polytropic flows: formal asymptotics in the periodic case", Stud. Appl. Math. 109 (2002), no. 2, p. 125-149.

[4] D. Bresch, B. Desjardins \& C.-K. Lin - "On some compressible fluid models: Korteweg, lubrication, and shallow water systems", Comm. Partial Differential Equations 28 (2003), no. 3-4, p. 843-868.

[5] C. Cheverry, O. Guès \& G. Métivier - "Oscillations fortes sur un champ linéairement dégénéré", Ann. Sci. École Norm. Sup. (4) 36 (2003), no. 5, p. 691745.

[6] R. DAnchin - "Global existence in critical spaces for compressible Navier-Stokes equations", Invent. Math. 141 (2000), no. 3, p. 579-614.

[7] _ "Global existence in critical spaces for flows of compressible viscous and heat-conductive gases", Arch. Ration. Mech. Anal. 160 (2001), no. 1, p. 1-39.

[8] _ "Zero Mach number limit for compressible flows with periodic boundary conditions", Amer. J. Math. 124 (2002), no. 6, p. 1153-1219.

[9] _ "Zero Mach number limit in critical spaces for compressible Navier-Stokes equations", Ann. Sci. École Norm. Sup. (4) 35 (2002), no. 1, p. $27-75$.

[10] B. Desjardins \& E. Grenier - "Low Mach number limit of viscous compressible flows in the whole space", R. Soc. Lond. Proc. Ser. A Math. Phys. Eng. Sci. 455 (1999), no. 1986, p. 2271-2279.

[11] B. Desjardins, E. Grenier, P.-L. Lions \& N. Masmoudi - "Incompressible limit for solutions of the isentropic Navier-Stokes equations with Dirichlet boundary conditions", J. Math. Pures Appl. (9) 78 (1999), no. 5, p. 461-471. 
[12] B. Desjardins \& C.-K. Lin - "A survey of the compressible Navier-Stokes equations", Taiwanese J. Math. 3 (1999), no. 2, p. 123-137.

[13] I. GALlaGHer - "Résultats récents sur la limite incompressible", Séminaire Bourbaki 2003-2004 926 (2003), no. 926.

[14] E. Grenier - "Oscillatory perturbations of the Navier-Stokes equations", J. Math. Pures Appl. (9) 76 (1997), no. 6, p. 477-498.

[15] D. R. VAN Der Heul, C. VUIK \& P. Wesseling - "A conservative pressurecorrection method for the Euler and ideal MHD equations at all speeds", Internat. J. Numer. Methods Fluids 40 (2002), no. 3-4, p. 521-529, ICFD Conference on Numerical Methods for Fluid Dynamics, Part II (Oxford, 2001).

[16] D. Hoff - "The zero-Mach limit of compressible flows", Comm. Math. Phys. 192 (1998), no. 3, p. 543-554.

[17] L. HÖRMANDER - Lectures on nonlinear hyperbolic differential equations, Mathématiques \& Applications, vol. 26, Springer-Verlag, Berlin, 1997.

[18] H. IsOzAKI - "Singular limits for the compressible Euler equation in an exterior domain", J. Reine Angew. Math. 381 (1987), p. 1-36.

[19] _ "Singular limits for the compressible Euler equation in an exterior domain. II. Bodies in a uniform flow", Osaka J. Math. 26 (1989), no. 2, p. 399-410.

[20] S. KaWAShima \& Y. Shizuta - "On the normal form of the symmetric hyperbolicparabolic systems associated with the conservation laws", Tohoku Math. J. (2) 40 (1988), no. 3, p. 449-464.

[21] S. KaWAshima \& W.-A. YONG - "Dissipative structure and entropy for hyperbolic systems of balance laws", Arch. Ration. Mech. Anal. (To appear), p. 1-20.

[22] S. Klainerman \& A. Majda - "Singular limits of quasilinear hyperbolic systems with large parameters and the incompressible limit of compressible fluids", Comm. Pure Appl. Math. 34 (1981), no. 4, p. 481-524.

[23] _ "Compressible and incompressible fluids", Comm. Pure Appl. Math. 35 (1982), no. 5, p. 629-651.

[24] R. KLEIN - "Semi-implicit extension of a Godunov-type scheme based on low Mach number asymptotics. I. One-dimensional flow", J. Comput. Phys. 121 (1995), no. 2, p. 213-237.

[25] D. LANNES - "Sharp estimates for pseudo-differential operators with symbols of limited smoothness and commutators", Preprint Bordeaux1 (2004), p. 1-31.

[26] P.-L. Lions - Mathematical topics in fluid mechanics. Vol. 1, Oxford Lecture Series in Mathematics and its Applications, vol. 3.

[27] P.-L. Lions \& N. MASmOud - "Incompressible limit for a viscous compressible fluid", J. Math. Pures Appl. (9) 77 (1998), no. 6, p. 585-627.

[28] A. MAJDA - Compressible fluid flow and systems of conservation laws in several space variables, Applied Mathematical Sciences, vol. 53, Springer-Verlag, New York, 1984.

[29] A. Matsumura \& T. Nishida - "The initial value problem for the equations of motion of viscous and heat-conductive gases", J. Math. Kyoto Univ. 20 (1980), no. 1, p. $67-104$.

[30] G. Métivier \& S. Schochet - "Limite incompressible des équations d'Euler non isentropiques", in Séminaire: Équations aux Dérivées Partielles, 2000-2001.

[31] _ "The incompressible limit of the non-isentropic Euler equations", Arch. Ration. Mech. Anal. 158 (2001), no. 1, p. 61-90.

[32] _ "Averaging theorems for conservative systems and the weakly compressible Euler equations", J. Differential Equations 187 (2003), no. 1, p. 106-183.

[33] Y. MeYER - "Remarques sur un théorème de J.-M. Bony", in Proceedings of the Seminar on Harmonic Analysis (Pisa, 1980), no. suppl. 1, 1981, p. 1-20.

[34] C.-D. Munz - "Computational fluid dynamics and aeroacoustics for low Mach number flow", in Hyperbolic partial differential equations (Hamburg, 2001), Vieweg, Braunschweig, 2002, p. 269-320. 
[35] T. Schneider, N. Botta, K. J. Geratz \& R. Klein - "Extension of finite volume compressible flow solvers to multi-dimensional, variable density zero Mach number flows", J. Comput. Phys. 155 (1999), no. 2, p. 248-286.

[36] S. Schochet - "The compressible Euler equations in a bounded domain: existence of solutions and the incompressible limit", Comm. Math. Phys. 104 (1986), no. 1, p. $49-75$.

[37] , "Fast singular limits of hyperbolic PDEs", J. Differential Equations 114 (1994), no. 2, p. 476-512.

[38] P. Secchi - "On slightly compressible ideal flow in the half-plane", Arch. Ration. Mech. Anal. 161 (2002), no. 3, p. 231-255.

[39] P. SECCHI - "On the singular incompressible limit of inviscid compressible fluids", J. Math. Fluid Mech. 2 (2000), no. 2, p. 107-125.

[40] M. E. TAYLOR - Pseudodifferential operators and nonlinear PDE, Progress in Mathematics, vol. 100, Birkhäuser Boston Inc., Boston, MA, 1991.

[41] S. UKAI - "The incompressible limit and the initial layer of the compressible Euler equation", J. Math. Kyoto Univ. 26 (1986), no. 2, p. 323-331.

[42] V. N. VAtsa \& E. TuRkel - "Choice of variables and preconditioning for time dependent problems", in AIAA, 16th Computational Fluid Dynamics Conference (Orlando), 2003.

[43] R. K. Zeytounian - Theory and applications of nonviscous fluid flows, SpringerVerlag, Berlin, 2002.

[44] _ Theory and applications of viscous fluid flows, Springer-Verlag, Berlin, 2004.

MAB Université de Bordeaux I, 33405 Talence Cedex, France

E-mail address: thomas.alazard@math.u-bordeaux1.fr 